\title{
Palladium-Catalyzed Domino Heck/Intermolecular C-H Bond \\ Functionalization: Efficient Synthesis of Alkylated Polyfluoroarene Derivatives
}

\author{
Xin-Xing Wu, Wen-Long Chen, Yi Shen, Si Chen, Peng-Fei Xu* and Yong-Min Liang* \\ State Key Laboratory of Applied Organic Chemistry, Lanzhou University, Lanzhou, \\ 730000, P.R. China. \\ E-mail: xupf@lzu.edu.cn; liangym@Izu.edu.cn
}

Contents

1. General Considerations......................................S2

2. Preparation of Starting Materials................................ 2

3. Experiment Procedure........................................S2

4. Spectra Data................................................. 14

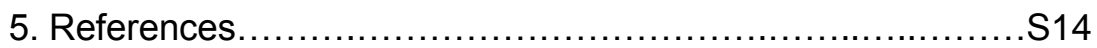

6. Crystallographic Data of $\mathbf{3 k}$ and $\mathbf{4 a} \ldots \ldots \ldots \ldots \ldots \ldots \ldots . . . . \ldots 15-\mathrm{S} 16$

7. Copies of ${ }^{1} \mathrm{H},{ }^{19} \mathrm{~F}$ and ${ }^{13} \mathrm{C}$ Spectra......................... $17-\mathrm{S} 61$ 


\section{General Considerations.}

Unless stated otherwise, all reactions were carried out in flame-dried glassware under a dry argon atmosphere. All solvents were purified and dried according to standard methods prior to use. For product purification by flash column chromatography, silica gel (200 300 mesh) and light petroleum ether (bp. 60 90) are used. ${ }^{1} \mathrm{H}$ NMR spectra were recorded on a Bruker advance III $400 \mathrm{MHz}$ in $\mathrm{CDCl}_{3}$ and ${ }^{13} \mathrm{C}$ NMR spectra were recorded on $100 \mathrm{MHz}$ in $\mathrm{CDCl}_{3}$ using TMS as internal standard, ${ }^{19} \mathrm{~F}$ NMR spectra were recorded on $376 \mathrm{MHz}$. Data for $1 \mathrm{H} \mathrm{NMR}$ are recorded as follows: chemical shift ( $\delta, \mathrm{ppm})$, multiplicity (s = singlet, $\mathrm{d}$ = doublet, $\mathrm{t}=$ triplet, $\mathrm{m}=$ multiplet or unresolved, $\mathrm{br}=$ broad singlet, coupling constant (s) in $\mathrm{Hz}$, integration). Data for $13 \mathrm{C}$ NMR is reported in terms of chemical shift $(\delta$, ppm). IR spectra were obtained on a Perkin-Elmer Model 2000 FT-IR using KBr plates (thin film). High-resolution mass spectral analysis (HRMS) data were measured on a Bruker Apex II.

\section{Preparation of Starting Materials.}

Compounds 1 were prepared according to the known procedures. ${ }^{1,2}$ Compounds 2 were commercially available.

\section{Experiment Procedure.}

1 (0.3 mmol), $\mathrm{Pd}(\mathrm{OAc})_{2}(5 \mathrm{~mol} \%)$, SPhos ( $\left.10 \mathrm{~mol} \%\right), \mathrm{Ag}_{2} \mathrm{CO}_{3}$ ( 0.75 equiv) were added to a sealed tube, EtOAc $(1 \mathrm{~mL})$ and $\mathrm{H}_{2} \mathrm{O}(0.5 \mathrm{~mL})$ were added via syringe. The mixture was flushed with $\mathrm{N}_{2}$, then $2(0.9 \mathrm{mmol})$ was added by syringe. The mixture was stirred at $75^{\circ} \mathrm{C}$ about for $24 \mathrm{~h}$ until completion (monitored by TLC). After cooling at room temperature, the solvent was removed under reduced pressure, the residue was purified by silica gel chromatography using PE/EA to afford the product $\mathbf{3}$ or $\mathbf{4}$. 


\section{Spectra Data.}

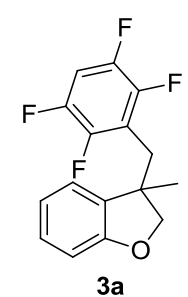

3-methyl-3-(2,3,5,6-tetrafluorobenzyl)-2,3-dihydrobenzofuran (3a): 79 mg; 89\% yield; colorless oil; ${ }^{1} \mathbf{H}$ NMR (400 MHz, $\mathbf{C D C l}_{3}$ ) $\delta$ 7.17-7.13 (m, $\left.1 \mathrm{H}\right), 7.02-6.86(\mathrm{~m}, 3 \mathrm{H}), 6.79-6.76$ $(\mathrm{m}, 1 \mathrm{H}), 4.52(\mathrm{~d}, J=9.2 \mathrm{~Hz}, 1 \mathrm{H}), 4.10(\mathrm{~d}, J=9.2 \mathrm{~Hz}, 1 \mathrm{H}), 3.10-3.00(\mathrm{~m}, 2 \mathrm{H}), 1.39(\mathrm{~s}, 3 \mathrm{H})$; ${ }^{13} \mathrm{C}$ NMR $\left(100 \mathrm{MHz}, \mathbf{C D C l}_{3}\right) \delta 159.3,145.7\left(\mathrm{dm}, J_{\mathrm{F}}=247.0 \mathrm{~Hz}\right), 145.0\left(\mathrm{dm}, J_{\mathrm{F}}=243.0\right.$ $\mathrm{Hz}), 133.4,133.4,133.3,128.7,122.9\left(\mathrm{t}, J_{F}=11.0 \mathrm{~Hz}\right), 120.7,117.4\left(\mathrm{t}, J_{F}=18.0 \mathrm{~Hz}\right)$, 109.8, 109.7, $104.5\left(\mathrm{t}, J_{\mathrm{F}}=17.5 \mathrm{~Hz}\right), 82.1\left(\mathrm{t}, J_{\mathrm{F}}=7.5 \mathrm{~Hz}\right), 46.7,33.2,23.8 ;{ }^{19} \mathrm{~F}$ NMR (376 $\mathbf{M H z}, \mathbf{C D C l}_{3}$ ) $\delta-139.3$ (m, 2F), -140.9 (m, 2F); IR (neat, $\mathbf{~ c m}^{-1}$ ): 3071, 2967, 1727, 1598, 1502, 1253, 1106, 983, 830, 752; HRMS (ESI) calcd for $\mathrm{C}_{16} \mathrm{H}_{13} \mathrm{~F}_{4} \mathrm{O}_{1}[\mathrm{M}+\mathrm{H}]^{+}:$: 297.0897, found: 297.0893 .

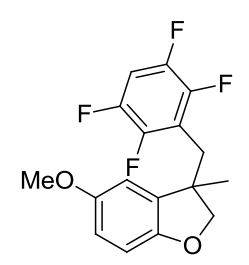

3b

5-methoxy-3-methyl-3-(2,3,5,6-tetrafluorobenzyl)-2,3-dihydrobenzofuran (3b): $77 \mathrm{mg}$; 79\% yield; colorless oil; ${ }^{1} \mathrm{H}$ NMR $\left(\mathbf{4 0 0} \mathbf{M H z}, \mathrm{CDCl}_{3}\right)$ ठ 6.99-6.91 (m, 1H), $6.68(\mathrm{~s}, 2 \mathrm{H}), 6.58$ (s, $1 \mathrm{H}), 4.48(\mathrm{~d}, J=8.8 \mathrm{~Hz}, 1 \mathrm{H}), 4.08(\mathrm{~d}, J=8.8 \mathrm{~Hz}, 1 \mathrm{H}), 3.73(\mathrm{~s}, 3 \mathrm{H}), 3.08-2.99(\mathrm{~m}, 2 \mathrm{H})$, $1.37(\mathrm{~s}, 3 \mathrm{H}) ;{ }^{13} \mathrm{C} \mathrm{NMR}\left(\mathbf{1 0 0} \mathbf{M H z}, \mathrm{CDCl}_{3}\right) \delta 154.2,153.3,145.6\left(\mathrm{dm}, J_{\mathrm{F}}=247.0 \mathrm{~Hz}\right), 145.0$ $\left(\mathrm{dm}, J_{\mathrm{F}}=243.0 \mathrm{~Hz}\right), 134.4,117.3\left(\mathrm{t}, J_{\mathrm{F}}=18.0 \mathrm{~Hz}\right), 113.6,109.8,109.0,104.5\left(\mathrm{t}, J_{\mathrm{F}}=23.0\right.$ $\mathrm{Hz}), 82.3,55.9,47.1,32.8,23.4 ;{ }^{19} \mathrm{~F}$ NMR (376 MHz, $\left.\mathbf{C D C l}_{3}\right) \delta-139.4(\mathrm{~m}, 2 \mathrm{~F}),-140.9(\mathrm{~m}$, 2F); IR (neat, $\mathbf{~ c m}^{-1}$ ): 3068, 2963, 1612, 1502, 1253, 1176, 1033, 984, 807, 713; HRMS (ESI) calcd for $\mathrm{C}_{17} \mathrm{H}_{15} \mathrm{~F}_{4} \mathrm{O}_{2}[\mathrm{M}+\mathrm{H}]^{+}: 327.1003$, found: 327.0999 .

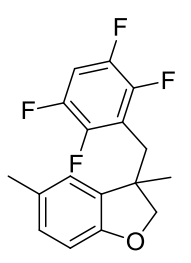

$3 c$

3,5-dimethyl-3-(2,3,5,6-tetrafluorobenzyl)-2,3-dihydrobenzofuran (3c): $74 \mathrm{mg} ; 80 \%$ yield; colorless oil; ' ${ }^{\mathbf{H}}$ NMR (400 MHz, $\left.\mathbf{C D C l}_{3}\right) \delta$ 6.96-6.88 (m, 2H), $6.81(\mathrm{~s}, 1 \mathrm{H}), 6.65(\mathrm{~d}, J$ $=8.0 \mathrm{~Hz}, 1 \mathrm{H}), 4.48(\mathrm{~d}, J=8.8 \mathrm{~Hz}, 1 \mathrm{H}), 4.05(\mathrm{~d}, J=8.8 \mathrm{~Hz}, 1 \mathrm{H}), 3.07-2.97(\mathrm{~m}, 2 \mathrm{H}), 2.26(\mathrm{~s}$, $3 \mathrm{H}), 1.35(\mathrm{~s}, 3 \mathrm{H}) ;{ }^{13} \mathrm{C}$ NMR (100 MHz, $\left.\mathbf{C D C l}_{3}\right) \delta$ 157.2, $145.7\left(\mathrm{dm}, \mathrm{J}_{\mathrm{F}}=246.0 \mathrm{~Hz}\right), 145.0$ $\left(\mathrm{dm}, J_{\mathrm{F}}=243.5 \mathrm{~Hz}\right), 133.6,129.9,129.0,123.3,117.5\left(\mathrm{t}, J_{\mathrm{F}}=18.0 \mathrm{~Hz}\right), 109.3,104.4\left(\mathrm{t}, J_{F}\right.$ 
$=22.5 \mathrm{~Hz}), 82.1\left(\mathrm{t}, J_{\mathrm{F}}=2.5 \mathrm{~Hz}\right), 46.7,33.0,23.5,20.7 ;{ }^{19} \mathrm{~F} \mathrm{NMR}\left(376 \mathrm{MHz}, \mathrm{CDCl}_{3}\right) \delta$ -139.4 (m, 2F), -140.9 (m, 2F); IR (neat, $\mathbf{~ c m}^{-1}$ ): 3067, 2968, 2879, 1613, 1502, 1253, 1173, 984, 840, 812, 714; HRMS (ESI) calcd for $\mathrm{C}_{17} \mathrm{H}_{15} \mathrm{~F}_{4} \mathrm{O}_{1}[\mathrm{M}+\mathrm{H}]^{+}: 311.1054$, found: 311.1048 .

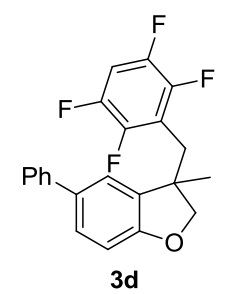

3-methyl-5-phenyl-3-(2,3,5,6-tetrafluorobenzyl)-2,3-dihydrobenzofuran (3d): $71 \mathrm{mg}$; 64\% yield; colorless oil; ${ }^{1} \mathrm{H}$ NMR $\left(\mathbf{4 0 0} \mathbf{~ M H z}, \mathbf{C D C l}_{3}\right)$ ठ 7.47-7.28 (m, 6H), $7.21(\mathrm{~s}, 1 \mathrm{H}), 6.94$ $(\mathrm{s}, 1 \mathrm{H}), 6.83(\mathrm{~d}, J=7.6 \mathrm{~Hz}, 1 \mathrm{H}), 4.56(\mathrm{~d}, J=8.4 \mathrm{~Hz}, 1 \mathrm{H}), 4.15(\mathrm{~d}, J=8.0 \mathrm{~Hz}, 1 \mathrm{H})$, 3.09-3.08 (m, 2H), $1.42(\mathrm{~s}, 3 \mathrm{H}) ;{ }^{13} \mathrm{C}$ NMR (100 MHz, $\left.\mathbf{C D C l}_{3}\right) \delta 159.0,145.7\left(\mathrm{dm}, J_{\mathrm{F}}=\right.$ $244.5 \mathrm{~Hz}), 145.0\left(\mathrm{dm}, J_{\mathrm{F}}=244.0 \mathrm{~Hz}\right), 141.3,134.4,134.1,128.7,127.9,126.8,126.6$, $121.8,117.3\left(\mathrm{t}, J_{\mathrm{F}}=18.0 \mathrm{~Hz}\right), 110.0,104.6\left(\mathrm{t}, J_{\mathrm{F}}=22.5 \mathrm{~Hz}\right), 82.5,46.8,33.2,23.9 ;{ }^{19} \mathrm{~F}$ NMR (376 MHz, $\mathbf{C D C l}_{3}$ ) $\delta-139.1$ (m, 2F), -140.7 (m, 2F); IR (neat, $\mathbf{~ c m}^{-1}$ ): 3066, 2928, 1729, 1612, 1501, 1248, 1181, 974, 774, 705; HRMS (ESI) calcd for $\mathrm{C}_{22} \mathrm{H}_{16} \mathrm{~F}_{4} \mathrm{O}_{1} \mathrm{Na}[\mathrm{M}+\mathrm{Na}]^{+}:$395.1029, found: 395.1011.

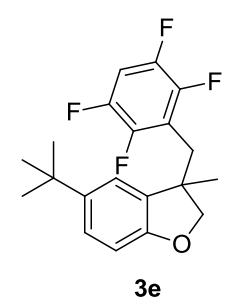

5-(tert-butyl)-3-methyl-3-(2,3,5,6-tetrafluorobenzyl)-2,3-dihydrobenzofuran (3e): 79 mg; 75\% yield; colorless oil; ${ }^{1} \mathbf{H}$ NMR $\left(400 \mathbf{~ M H z}, \mathbf{C D C l}_{3}\right) \delta 7.16(\mathrm{~d}, J=8.4 \mathrm{~Hz}, 1 \mathrm{H})$, 6.96-6.88 (m, 1H), $6.85(\mathrm{~s}, 1 \mathrm{H}), 6.70(\mathrm{~d}, J=8.4 \mathrm{~Hz}, 1 \mathrm{H}), 4.50(\mathrm{~d}, J=8.8 \mathrm{~Hz}, 1 \mathrm{H}), 4.13(\mathrm{~d}$, $J=8.8 \mathrm{~Hz}, 1 \mathrm{H}), 3.09-2.97(\mathrm{~m}, 2 \mathrm{H}), 1.41(\mathrm{~s}, 3 \mathrm{H}), 1.25(\mathrm{~s}, 9 \mathrm{H}) ;{ }^{13} \mathrm{C} \mathrm{NMR}\left(100 \mathrm{MHz}, \mathrm{CDCl}_{3}\right)$ $\delta 157.1,145.6\left(\mathrm{dm}, J_{F}=246.0 \mathrm{~Hz}\right), 145.0\left(\mathrm{dm}, J_{F}=254.0 \mathrm{~Hz}\right), 132.5,125.4,119.7,117.5$ $\left(\mathrm{t}, J_{F}=18.0 \mathrm{~Hz}\right), 108.9,104.3\left(\mathrm{t}, J_{\mathrm{F}}=22.5 \mathrm{~Hz}\right), 46.9,34.3,33.3,31.5,23.3 ;{ }^{19} \mathrm{~F}$ NMR (376 MHz, $\mathbf{C D C l}_{3}$ ) $\delta-139.5$ (m, 2F), -140.8 (m, 2F); IR (neat, cm ${ }^{-1}$ ): 2966, 1614, 1503, 1364, 1254, 1173, 981, 840, 820, 740; HRMS (ESI) calcd for $\mathrm{C}_{20} \mathrm{H}_{20} \mathrm{~F}_{4} \mathrm{O}_{1} \mathrm{Na}[\mathrm{M}+\mathrm{Na}]^{+}: 375.1342$, found: 375.1360 .

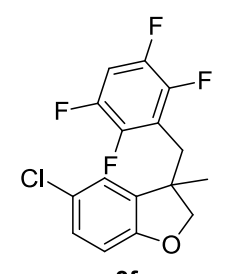

$3 \mathbf{f}$

5-chloro-3-methyl-3-(2,3,5,6-tetrafluorobenzyl)-2,3-dihydrobenzofuran (3f): 76 mg; $77 \%$ yield; colorless solid; $\mathrm{mp}=85-87^{\circ} \mathrm{C} ;{ }^{1} \mathrm{H}$ NMR $\left(400 \mathbf{M H z} \mathrm{CDCl}_{3}\right) \delta 7.08$ (dd, $\mathrm{J}=8.4$ 
$\mathrm{Hz}, J=2.4 \mathrm{~Hz}, 1 \mathrm{H}), 7.02-6.93(\mathrm{~m}, 2 \mathrm{H}), 6.68(\mathrm{~d}, J=8.8 \mathrm{~Hz}, 1 \mathrm{H}), 4.53(\mathrm{~d}, J=8.8 \mathrm{~Hz}, 1 \mathrm{H})$, $4.12(\mathrm{~d}, J=9.2 \mathrm{~Hz}, 1 \mathrm{H}), 3.03(\mathrm{q}, J=9.6 \mathrm{~Hz}, 2 \mathrm{H}), 1.38(\mathrm{~s}, 3 \mathrm{H}) ;{ }^{13} \mathrm{C} \mathrm{NMR}\left(100 \mathrm{MHz}, \mathrm{CDCl}_{3}\right)$ $\delta$ 157.9, $145.7\left(\mathrm{dm}, J_{\mathrm{F}}=246.0 \mathrm{~Hz}\right), 145.0\left(\mathrm{dm}, J_{\mathrm{F}}=244.0 \mathrm{~Hz}\right), 135.4,128.5,125.3,123.1$, $116.9\left(\mathrm{t}, J_{F}=18.0 \mathrm{~Hz}\right), 110.9,104.8\left(\mathrm{t}, J_{\mathrm{F}}=22.5 \mathrm{~Hz}\right), 82.5\left(\mathrm{t}, J_{\mathrm{F}}=2.5 \mathrm{~Hz}\right), 46.9$, 33.0, 23.6; ${ }^{19} \mathrm{~F}$ NMR (376 MHz, $\mathbf{C D C l}_{3}$ ) $\delta-139.0$ (m, 2F), -141.0 (m, 2F); IR (neat, $\mathbf{~ c m}^{-1}$ ): 3049, 2975, $1864,1753,1613,1300,1182,978,813,713$; HRMS (ESI) calcd for $\mathrm{C}_{16} \mathrm{H}_{12} \mathrm{~F}_{4} \mathrm{O}_{1} \mathrm{CI}[\mathrm{M}+\mathrm{H}]^{+}$: 331.0507, found: 331.0505 .

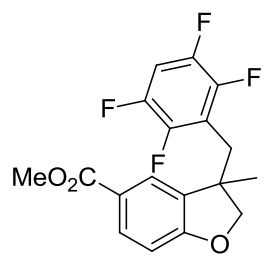

$3 \mathbf{g}$

methyl 3-methyl-3-(2,3,5,6-tetrafluorobenzyl)-2,3-dihydrobenzofuran-5-carboxylate (3g): 69 mg; 65\% yield; colorless oil; ${ }^{1} \mathbf{H}$ NMR (400 MHz, $\left.\mathbf{C D C l}_{3}\right) \delta 7.90(\mathrm{~d}, J=8.0 \mathrm{~Hz}, 1 \mathrm{H})$, $7.75(\mathrm{~s}, 1 \mathrm{H}), 7.00-6.97(\mathrm{~m}, 2 \mathrm{H}), 6.77(\mathrm{~d}, J=8.0 \mathrm{~Hz}, 1 \mathrm{H}), 4.62(\mathrm{~d}, J=8.8 \mathrm{~Hz}, 1 \mathrm{H}), 4.19$ (d, $J=8.8 \mathrm{~Hz}, 1 \mathrm{H}), 3.88(\mathrm{~s}, 3 \mathrm{H}), 3.05(\mathrm{q}, J=12.8 \mathrm{~Hz}, 2 \mathrm{H}), 1.43(\mathrm{~s}, 3 \mathrm{H}) ;{ }^{13} \mathrm{C}$ NMR (100 MHz, $\left.\mathrm{CDCl}_{3}\right) \delta 166.6,163.3,145.7\left(\mathrm{dm}, J_{\mathrm{F}}=247.0 \mathrm{~Hz}\right), 144.9\left(\mathrm{dm}, J_{\mathrm{F}}=244.0 \mathrm{~Hz}\right), 133.9,131.5$, 124.8, 122.9, $116.8\left(\mathrm{t}, J_{\mathrm{F}}=18.0 \mathrm{~Hz}\right), 109.4,104.7\left(\mathrm{t}, J_{\mathrm{F}}=23.0 \mathrm{~Hz}\right), 82.9,51.7,46.2,33.1$, 23.8; ${ }^{19} \mathrm{~F}$ NMR (376 MHz, $\mathbf{C D C l}_{3}$ ) $\delta-139.1$ (m, 2F), -141.0 (m, 2F); IR (neat, $\mathbf{~ c m}^{-1}$ ): 2959, 1716, 1612, 1503, 1439, 1288, 1253, 1103, 979, 839, 773; HRMS (ESI) calcd for $\mathrm{C}_{18} \mathrm{H}_{14} \mathrm{~F}_{4} \mathrm{O}_{3} \mathrm{Na}[\mathrm{M}+\mathrm{Na}]^{+}: 377.0771$, found: 377.0771 .

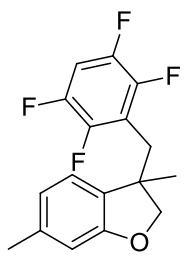

3h

3,6-dimethyl-3-(2,3,5,6-tetrafluorobenzyl)-2,3-dihydrobenzofuran (3h): $79 \mathrm{mg} ; 85 \%$ yield; colorless oil; ${ }^{1} \mathbf{H}$ NMR (400 $\mathbf{~ M H z}, \mathbf{C D C l}_{3}$ ) $\delta$ 6.96-6.87 (m, 2H), $6.68(\mathrm{~d}, J=7.6 \mathrm{~Hz}$, $1 \mathrm{H}), 6.59(\mathrm{~s}, 1 \mathrm{H}), 4.49(\mathrm{~d}, J=8.8 \mathrm{~Hz}, 1 \mathrm{H}), 4.07(\mathrm{~d}, J=8.8 \mathrm{~Hz}, 1 \mathrm{H}), 3.04(\mathrm{q}, J=13.2 \mathrm{~Hz}$, $2 \mathrm{H}), 2.29(\mathrm{~s}, 3 \mathrm{H}), 1.36(\mathrm{~s}, 3 \mathrm{H}) ;{ }^{13} \mathrm{C}$ NMR (100 MHz, $\left.\mathbf{C D C l}_{3}\right) \delta$ 159.5, $145.7\left(\mathrm{dm}, \mathrm{J}_{\mathrm{F}}=246.0\right.$ $\mathrm{Hz}), 145.0\left(\mathrm{dm}, J_{\mathrm{F}}=243.0 \mathrm{~Hz}\right), 138.9,130.6,122.3,121.4,117.5\left(\mathrm{t}, J_{\mathrm{F}}=18.0 \mathrm{~Hz}\right), 110.5$, $104.4\left(\mathrm{t}, J_{\mathrm{F}}=22.0 \mathrm{~Hz}\right), 82.3\left(\mathrm{t}, J_{\mathrm{F}}=2.5 \mathrm{~Hz}\right), 46.4,33.1,23.9,21.4 ;{ }^{19} \mathrm{~F}$ NMR (376 MHz, $\mathbf{C D C l}_{3}$ ) $\delta-139.3(\mathrm{~m}, 2 \mathrm{~F}),-140.8$ (m, 2F); IR (neat, $\mathbf{c m}^{-1}$ ): 2967, 1620, 1501, 1453, 1253, 1172, 1008, 841, 713, 596; HRMS (ESI) calcd for $\mathrm{C}_{17} \mathrm{H}_{14} \mathrm{~F}_{4} \mathrm{O}_{1} \mathrm{Na}[\mathrm{M}+\mathrm{Na}]^{+}:$333.0873, found: 333.0881 . 


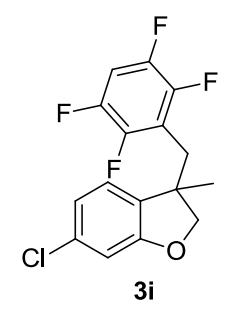

6-chloro-3-methyl-3-(2,3,5,6-tetrafluorobenzyl)-2,3-dihydrobenzofuran (3i): $55 \mathrm{mg}$; $56 \%$ yield; colorless oil; ${ }^{1} \mathbf{H}$ NMR $\left(\mathbf{4 0 0} \mathbf{~ M H z}, \mathbf{C D C l}_{3}\right) \delta 7.01-6.94(\mathrm{~m}, 1 \mathrm{H}), 6.90(\mathrm{~d}, J=8.0$ $\mathrm{Hz}, 1 \mathrm{H}), 6.84(\mathrm{dd}, J=8.0 \mathrm{~Hz}, J=1.2 \mathrm{~Hz}, 1 \mathrm{H}), 6.76(\mathrm{~s}, 1 \mathrm{H}), 4.54(\mathrm{~d}, J=9.2 \mathrm{~Hz}, 1 \mathrm{H}), 4.14$ (d, $J=8.8 \mathrm{~Hz}, 1 \mathrm{H}), 3.03(\mathrm{q}, J=10.8 \mathrm{~Hz}, 2 \mathrm{H}), 1.38(\mathrm{~s}, 3 \mathrm{H}) ;{ }^{13} \mathrm{C} \mathrm{NMR}\left(100 \mathrm{MHz}, \mathrm{CDCl}_{3}\right) \delta$ $160.2,145.7\left(\mathrm{dm}, J_{F}=247.0 \mathrm{~Hz}\right), 144.9\left(\mathrm{dm}, J_{F}=234.0 \mathrm{~Hz}\right), 134.0,132.2,123.5,120.8$, $117.0\left(\mathrm{t}, J_{\mathrm{F}}=19.0 \mathrm{~Hz}\right), 110.6,104.7\left(\mathrm{t}, J_{\mathrm{F}}=22.0 \mathrm{~Hz}\right), 82.9\left(\mathrm{t}, J_{\mathrm{F}}=2.5 \mathrm{~Hz}\right), 46.4,33.1,23.9$; ${ }^{19} \mathrm{~F}$ NMR (376 MHz, $\mathbf{C D C l}_{3}$ ) $\delta-139.0$ (m, 2F), -140.8 (m, 2F); IR (neat, $\mathbf{c m}^{-1}$ ): 2969, 1603, 1501, 1420, 1253, 1173, 1076, 982, 843, 713; HRMS (ESI) calcd for $\mathrm{C}_{16} \mathrm{H}_{12} \mathrm{~F}_{4} \mathrm{O}_{1} \mathrm{Cl}[\mathrm{M}+\mathrm{H}]^{+}$: 331.0507, found: 331.0508 .

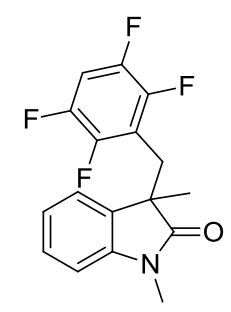

3j

1,3-dimethyl-3-(2,3,5,6-tetrafluorobenzyl)indolin-2-one (3j): $76 \mathrm{mg} ; 78 \%$ yield; yellow solid; $\mathrm{mp}=86-88{ }^{\circ} \mathrm{C} ;{ }^{1} \mathbf{H}$ NMR $\left(400 \mathrm{MHz}, \mathbf{C D C l}_{3}\right) \delta$ 7.26-7.22 (m, $\left.1 \mathrm{H}\right), 7.03-7.00(\mathrm{~m}, 2 \mathrm{H})$, 6.93-6.87 (m, 1H), $6.80(\mathrm{~d}, J=7.2 \mathrm{~Hz}, 1 \mathrm{H}), 3.21(\mathrm{~s}, 3 \mathrm{H}), 3.17(\mathrm{~s}, 2 \mathrm{H}), 1.46(\mathrm{~s}, 3 \mathrm{H}) ;{ }^{13} \mathrm{C}$ NMR $\left(100 \mathrm{MHz}, \mathrm{CDCl}_{3}\right) \delta 178.9,145.4\left(\mathrm{dm}, J_{\mathrm{F}}=251.0 \mathrm{~Hz}\right), 144.9\left(\mathrm{dm}, J_{\mathrm{F}}=243.0 \mathrm{~Hz}\right)$, 142.6, 132.2, 128.2, 122.8, 122.2, 116.2 (t, $\left.J_{F}=18.0 \mathrm{~Hz}\right), 107.9,104.6$ (t, $J_{F}=22.0 \mathrm{~Hz}$ ), 47.7, 31.1, 26.1, 22.0; ${ }^{19} \mathrm{~F}$ NMR (376 $\mathbf{~ M H z}, \mathbf{C D C l}_{3}$ ) $\delta-139.6$ (m, 2F), -140.1 (m, 2F); IR (neat, $\mathbf{c m}^{-1}$ ): 2928, 1719, 1613, 1504, 1378, 1254, 1100, 966, 753; HRMS (ESI) calcd for $\mathrm{C}_{17} \mathrm{H}_{14} \mathrm{~F}_{4} \mathrm{O}_{1} \mathrm{~N}[\mathrm{M}+\mathrm{H}]^{+}:$324.1006, found: 324.1006.

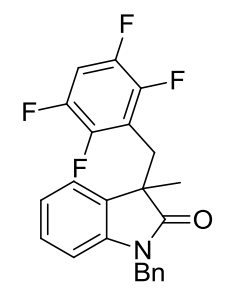

3k

1-benzyl-3-methyl-3-(2,3,5,6-tetrafluorobenzyl)indolin-2-one (3k): 105 mg; 88\% yield; yellow solid; $\mathrm{mp}=87-89{ }^{\circ} \mathrm{C}$; ${ }^{1} \mathrm{H}$ NMR (400 MHz, $\left.\mathbf{C D C l}_{3}\right) \delta$ 7.26-7.21 (m, 5H), 7.12-7.06 (m, 2H), 7.00-6.94 (m, $1 \mathrm{H}), 6.90-6.84(\mathrm{~m}, 1 \mathrm{H}), 6.66(\mathrm{~d}, J=7.6 \mathrm{~Hz}, 1 \mathrm{H}), 5.00(\mathrm{~d}, J=15.6 \mathrm{~Hz}$, $1 \mathrm{H}), 4.74(\mathrm{~d}, J=15.6 \mathrm{~Hz}, 1 \mathrm{H}), 3.24(\mathrm{q}, J=16.0 \mathrm{~Hz}, 2 \mathrm{H}), 1.51(\mathrm{~s}, 3 \mathrm{H}) ;{ }^{13} \mathrm{C} \mathrm{NMR}(\mathbf{1 0 0} \mathbf{~ M H z}$, $\left.\mathrm{CDCl}_{3}\right) \delta 178.7,145.4\left(\mathrm{dm}, J_{\mathrm{F}}=248.5 \mathrm{~Hz}\right), 144.8\left(\mathrm{dm}, J_{\mathrm{F}}=243.5 \mathrm{~Hz}\right), 141.7,135.7,132.2$, $128.5,128.1,127.4,127.0,123.0,122.3,116.1\left(\mathrm{t}, J_{F}=18.0 \mathrm{~Hz}\right), 108.9,104.6\left(\mathrm{t}, J_{F}=23.0\right.$ 
$\mathrm{Hz}), 47.8,43.5,31.0,22.7 ;{ }^{19} \mathbf{F}$ NMR (376 MHz, $\left.\mathbf{C D C l}_{3}\right) \delta-139.3(\mathrm{~m}, 2 \mathrm{~F}),-139.5(\mathrm{~m}, 2 \mathrm{~F})$; IR (neat, $\mathbf{~ c m}^{-1}$ ): 2926, 1717, 1612, 1502, 1455, 1358, 1254, 1177, 1105, 879, 747, 699; HRMS (ESI) calcd for $\mathrm{C}_{23} \mathrm{H}_{18} \mathrm{~F}_{4} \mathrm{O}_{1} \mathrm{~N}[\mathrm{M}+\mathrm{H}]^{+}: 400.1319$, found: 400.1315 .

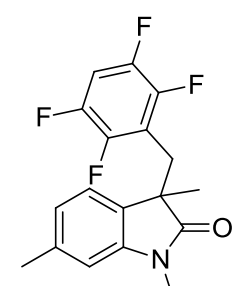

3)

1,3,6-trimethyl-3-(2,3,5,6-tetrafluorobenzyl)indolin-2-one (3l): $73 \mathrm{mg} ; 72 \%$ yield;

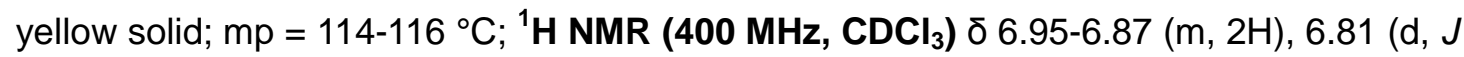
$=7.2 \mathrm{~Hz}, 1 \mathrm{H}), 6.64(\mathrm{~s}, 1 \mathrm{H}), 3.19(\mathrm{~s}, 3 \mathrm{H}), 3.15(\mathrm{~s}, 2 \mathrm{H}), 2.35(\mathrm{~s}, 3 \mathrm{H}), 1.43(\mathrm{~s}, 3 \mathrm{H}) ;{ }^{13} \mathrm{C} \mathrm{NMR}$ $\left(100 \mathrm{MHz}, \mathrm{CDCl}_{3}\right) \delta 179.2,145.4\left(\mathrm{dm}, J_{\mathrm{F}}=247.5 \mathrm{~Hz}\right), 144.9\left(\mathrm{dm}, J_{\mathrm{F}}=235.5 \mathrm{~Hz}\right), 142.7$, 138.3, 129.4, 122.7, 122.6, $116.4\left(\mathrm{t}, J_{\mathrm{F}}=18.0 \mathrm{~Hz}\right), 108.9,104.6\left(\mathrm{t}, J_{\mathrm{F}}=22.0 \mathrm{~Hz}\right), 47.5$, 31.1, 26.0, 22.1, 21.6; ${ }^{19} \mathrm{~F}$ NMR (376 $\mathbf{M H z}, \mathbf{C D C l}_{3}$ ) $\delta-139.7$ (m, 2F), -140.1 (m, 2F); IR (neat, $\mathbf{c m}^{-1}$ ): 2932, 1720, 1621, 1502, 1381, 1254, 1173, 1037, 838, 715; HRMS (ESI) calcd for $\mathrm{C}_{18} \mathrm{H}_{16} \mathrm{~F}_{4} \mathrm{O}_{1} \mathrm{~N}[\mathrm{M}+\mathrm{H}]^{+}:$338.1163, found: 338.1169 .

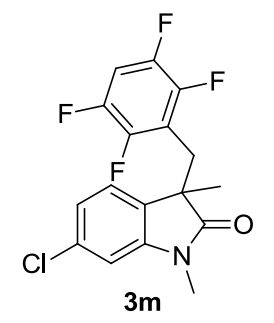

6-chloro-1,3-dimethyl-3-(2,3,5,6-tetrafluorobenzyl)indolin-2-one (3m): 64 mg; 60\% yield; yellow solid; $\mathrm{mp}=130-132{ }^{\circ} \mathrm{C}$; ${ }^{1} \mathbf{H}$ NMR $\left(\mathbf{4 0 0} \mathbf{~ M H z}, \mathbf{C D C l}_{3}\right)$ ठ 6.99-6.89 (m, 3H), 6.80 (s, 1H), $3.18(\mathrm{~s}, 3 \mathrm{H}), 3.16(\mathrm{~s}, 2 \mathrm{H}), 1.45(\mathrm{~s}, 3 \mathrm{H}) ;{ }^{13} \mathbf{C ~ N M R}\left(\mathbf{1 0 0 ~ M H z}, \mathbf{C D C l}_{3}\right) \delta$ 178.9, 145.6 $\left(\mathrm{dm}, J_{\mathrm{F}}=246.0 \mathrm{~Hz}\right), 144.9\left(\mathrm{dm}, J_{F}=244.0 \mathrm{~Hz}\right), 144.0,134.1,130.7,123.9,122.1,116.0(\mathrm{t}$, $\left.J_{F}=18.0 \mathrm{~Hz}\right), 108.7,104.9\left(\mathrm{t}, J_{\mathrm{F}}=23.0 \mathrm{~Hz}\right), 47.6,31.1,26.3,22.2 ;{ }^{19} \mathrm{~F}$ NMR (376 MHz, $\mathbf{C D C l}_{3}$ ) $\delta-139.3(\mathrm{~m}, 2 \mathrm{~F}),-140.1$ (m, 2F); IR (neat, $\mathbf{c m}^{-1}$ ): 2927, 1719, 1609, 1501, 1252, 1108, 842; HRMS (ESI) calcd for $\mathrm{C}_{17} \mathrm{H}_{13} \mathrm{~F}_{4} \mathrm{O}_{1} \mathrm{NCI}[\mathrm{M}+\mathrm{H}]^{+}:$358.0616, found: 358.0619 .<smiles>CCC1(Cc2c(F)cc(F)cc2F)C(=O)N(C)c2ccc(Cl)cc21</smiles>

5-chloro-1,3-dimethyl-3-(2,3,5,6-tetrafluorobenzyl)indolin-2-one (3n): 69 mg; 65\% yield; yellow solid; $\mathrm{mp}=138-140{ }^{\circ} \mathrm{C}$; ${ }^{1} \mathrm{H}$ NMR $\left(400 \mathrm{MHz}, \mathrm{CDCl}_{3}\right) \delta 7.23$ (dd, J=8.4 Hz, J $=2.0 \mathrm{~Hz}, 1 \mathrm{H}), 7.03-6.92(\mathrm{~m}, 2 \mathrm{H}), 6.74(\mathrm{~d}, J=8.4 \mathrm{~Hz}, 1 \mathrm{H}), 3.20(\mathrm{~s}, 3 \mathrm{H}), 3.15(\mathrm{~s}, 2 \mathrm{H}), 1.46$ (s, 3H); ${ }^{13} \mathrm{C}$ NMR (100 MHz, $\left.\mathbf{C D C l}_{3}\right) \delta 178.3,145.5\left(\mathrm{dm}, J_{\mathrm{F}}=246.0 \mathrm{~Hz}\right), 144.9\left(\mathrm{dm}, J_{\mathrm{F}}=\right.$ $245.0 \mathrm{~Hz}), 141.2,134.0,128.1,127.7,123.5,115.8\left(\mathrm{t}, J_{\mathrm{F}}=18.0 \mathrm{~Hz}\right), 108.9,105.0\left(\mathrm{t}, J_{F}=\right.$ 
23.0 Hz), 48.0, 31.0, 26.3, 21.8; ${ }^{19} \mathrm{~F}$ NMR (376 MHz, $\left.\mathbf{C D C l}_{3}\right) \delta-139.3(\mathrm{~m}, 2 \mathrm{~F}),-140.2(\mathrm{~m}$, 2F); IR (neat, $\mathbf{c m}^{-1}$ ): 2935, 1720, 1611, 1462, 1348, 1255, 1056, 888, 648; HRMS (ESI) calcd for $\mathrm{C}_{17} \mathrm{H}_{12} \mathrm{~F}_{4} \mathrm{O}_{1} \mathrm{NCINa}[\mathrm{M}+\mathrm{Na}]^{+}: 380.0436$, found: 380.0445 .

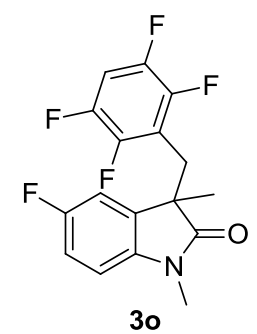

5-fluoro-1,3-dimethyl-3-(2,3,5,6-tetrafluorobenzyl)indolin-2-one (3o): $58 \mathrm{mg}$; 57\% yield; colorless oil; ${ }^{1} \mathbf{H}$ NMR (400 MHz, $\left.\mathbf{C D C l}_{3}\right) \delta 6.97-6.90(\mathrm{~m}, 2 \mathrm{H}), 6.80(\mathrm{~d}, J=7.2 \mathrm{~Hz}, 1 \mathrm{H})$, $6.73(\mathrm{dd}, J=8.4 \mathrm{~Hz}, J=4.0 \mathrm{~Hz}, 1 \mathrm{H}), 3.19(\mathrm{~s}, 3 \mathrm{H}), 3.17(\mathrm{~s}, 2 \mathrm{H}), 1.46(\mathrm{~s}, 3 \mathrm{H}) ;{ }^{13} \mathrm{C}$ NMR $\left(\mathbf{1 0 0 ~ M H z}, \mathrm{CDCl}_{3}\right) \delta 178.6,160.2,157.9,145.6\left(\mathrm{dm}, J_{\mathrm{F}}=245.5 \mathrm{~Hz}\right), 145.0\left(\mathrm{dm}, J_{\mathrm{F}}=244.0\right.$ $\mathrm{Hz}), 138.7,138.7,134.1,134.0,116.0\left(\mathrm{t}, J_{F}=18.0 \mathrm{~Hz}\right), 114.5,114.3,111.3,111.0,108.5$, 108.4, $104.9\left(\mathrm{t}, J_{\mathrm{F}}=23.0 \mathrm{~Hz}\right), 48.3,31.1,26.3,22.1 ;{ }^{19} \mathbf{F} \mathbf{N M R}\left(376 \mathbf{~ M H z}, \mathrm{CDCl}_{3}\right) \delta-120.7$ (d, $J=2.6 \mathrm{~Hz}, 1 \mathrm{~F}),-139.4$ (m, 2F), -140.2 (m, 2F); IR (neat, cm $\left.{ }^{-1}\right):$ 2931, 1720, 1459, 1351, 1255, 1173, 1116, 812, 714; HRMS (ESI) calcd for $\mathrm{C}_{17} \mathrm{H}_{13} \mathrm{~F}_{5} \mathrm{O}_{1} \mathrm{~N}[\mathrm{M}+\mathrm{H}]^{+}: 342.0912$, found: 342.0919 .

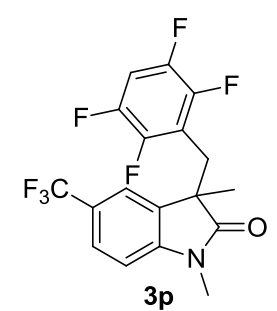

1,3-dimethyl-3-(2,3,5,6-tetrafluorobenzyl)-5-(trifluoromethyl)indolin-2-one (3p): 59 mg; $51 \%$ yield; yellow solid; $\mathrm{mp}=106-108{ }^{\circ} \mathrm{C} ;{ }^{1} \mathrm{H}$ NMR $\left(400 \mathrm{MHz}, \mathrm{CDCl}_{3}\right) \delta 7.55(\mathrm{~d}, \mathrm{~J}=$ $8.0 \mathrm{~Hz}, 1 \mathrm{H}), 7.23(\mathrm{~s}, 1 \mathrm{H}), 7.00-6.88(\mathrm{~m}, 2 \mathrm{H}), 3.25(\mathrm{~s}, 3 \mathrm{H}), 3.18(\mathrm{~s}, 2 \mathrm{H}), 1.50(\mathrm{~s}, 3 \mathrm{H}) ;{ }^{13} \mathrm{C}$ NMR (100 MHz, $\left.\mathbf{C D C l}_{3}\right) \delta 178.9,145.6\left(\mathrm{dm}, J_{F}=246.0 \mathrm{~Hz}\right), 145.7,144.9\left(\mathrm{dm}, J_{\mathrm{F}}=244.5\right.$ $\mathrm{Hz}), 132.9,126.1,126.1,126.0,125.6,124.6\left(q, J_{F}=32.0 \mathrm{~Hz}\right), 122.9,120.1,120.1$, 115.7 (t, $\left.J_{F}=18.0 \mathrm{~Hz}\right), 107.7,105.1\left(\mathrm{t}, J_{\mathrm{F}}=23.0 \mathrm{~Hz}\right), 47.8,31.1,26.4,21.8 ;{ }^{19} \mathrm{~F}$ NMR (376 MHz, $\mathbf{C D C l}_{3}$ ) $\delta-61.7$ (s, 3F), -139.3 (m, 2F), -140.3 (m, 2F); IR (neat, $\mathbf{c m}^{-1}$ ): 3064, 2930, 1728, 1625, 1503, 1460, 1329, 1120, 1033, 895, 714; HRMS (ESI) calcd for $\mathrm{C}_{18} \mathrm{H}_{13} \mathrm{~F}_{7} \mathrm{NO}_{1}[\mathrm{M}+\mathrm{H}]^{+}:$:392.0880, found: 392.0888 .

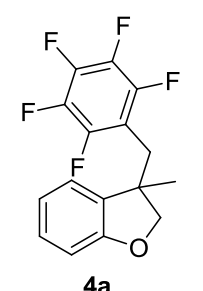

3-methyl-3-((perfluorophenyl)methyl)-2,3-dihydrobenzofuran (4a): 75 mg; 80\% yield; 
colorless solid; mp = 30-32 ${ }^{\circ} \mathrm{C} ;{ }^{1} \mathbf{H}$ NMR $\left(400 \mathbf{M H z} \mathbf{C D C l}_{3}\right) \delta 7.16-7.11(\mathrm{~m}, 1 \mathrm{H}), 6.98(\mathrm{~d}, \mathrm{~J}$ $=7.2 \mathrm{~Hz}, 1 \mathrm{H}), 6.86(\mathrm{t}, J=7.6 \mathrm{~Hz}, 1 \mathrm{H}), 6.75(\mathrm{~d}, J=8.0 \mathrm{~Hz}, 1 \mathrm{H}), 4.48(\mathrm{~d}, J=8.8 \mathrm{~Hz}, 1 \mathrm{H})$, $4.10(\mathrm{~d}, J=8.8 \mathrm{~Hz}, 1 \mathrm{H}), 3.00(\mathrm{q}, J=13.6 \mathrm{~Hz}, 2 \mathrm{H}), 1.40(\mathrm{~s}, 3 \mathrm{H}) ;{ }^{13} \mathrm{C}$ NMR (100 MHz, $\left.\mathrm{CDCl}_{3}\right) \delta 159.3,145.4\left(\mathrm{dm}, J_{\mathrm{F}}=250.0 \mathrm{~Hz}\right), 139.9\left(\mathrm{dm}, J_{\mathrm{F}}=263.0 \mathrm{~Hz}\right), 137.3\left(\mathrm{dm}, J_{\mathrm{F}}=\right.$ 251.0 Hz), 133.0, 128.8, 122.8, 120.7, 111.6-111.2 (m), 109.9, 82.1, 82.1, 46.6, 32.7, 23.6; ${ }^{19}$ F NMR (376 MHz, $\mathbf{C D C l}_{3}$ ) $\delta-140.4(\mathrm{~m}, 2 \mathrm{~F}),-156.0(\mathrm{~m}, 1 \mathrm{~F}),-162.5(\mathrm{~m}, 2 \mathrm{~F})$; IR (neat, $\left.\mathbf{c m}^{-1}\right)$ : 2969, 1598, 1522, 1502, 1232, 1123, 991, 972, 752; HRMS (ESI) calcd for $\mathrm{C}_{16} \mathrm{H}_{12} \mathrm{~F}_{5} \mathrm{O}_{1}[\mathrm{M}+\mathrm{H}]^{+}:$315.0807, found: 315.0799.

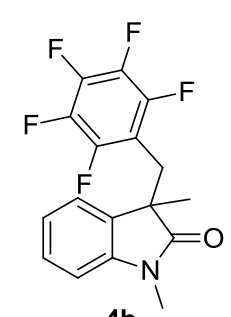

4b

1,3-dimethyl-3-((perfluorophenyl)methyl)indolin-2-one (4b): $88 \mathrm{mg} ; 86 \%$ yield; yellow solid; mp = 92-94 ${ }^{\circ} \mathrm{C} ;{ }^{1} \mathbf{H}$ NMR (400 MHz, $\left.\mathbf{C D C l}_{3}\right) \delta$ 7.28-7.24 (m, 1H), 7.09-7.01 (m, $\left.1 \mathrm{H}\right)$, $6.82(\mathrm{~d}, J=7.6 \mathrm{~Hz}, 1 \mathrm{H}), 3.21(\mathrm{~s}, 3 \mathrm{H}), 3.14(\mathrm{~m}, 2 \mathrm{H}), 1.45(\mathrm{~s}, 3 \mathrm{H}) ;{ }^{13} \mathrm{C}$ NMR (100 MHz, $\left.\mathrm{CDCl}_{3}\right) \delta 178.7,145.2\left(\mathrm{dm}, J_{\mathrm{F}}=246.0 \mathrm{~Hz}\right), 142.6,139.8\left(\mathrm{dm}, J_{\mathrm{F}}=271.0 \mathrm{~Hz}\right), 137.1(\mathrm{dm}$, $\left.J_{F}=265.0 \mathrm{~Hz}\right), 132.2,128.3,122.7,122.3,110.5-110.1(\mathrm{~m}), 108.0,47.6,30.6,26.1,21.9$; ${ }^{19} \mathrm{~F}$ NMR (376 MHz, $\left.\mathbf{C D C l}_{3}\right) \delta-139.5(\mathrm{~m}, 2 \mathrm{~F}),-155.8(\mathrm{~m}, 1 \mathrm{~F}),-162.7(\mathrm{~m}, 2 \mathrm{~F})$; IR (neat, $\mathbf{c m}^{-1}$ ): 2933, 1719, 1614, 1522, 1504, 1379, 1122, 986, 755; HRMS (ESI) calcd for $\mathrm{C}_{17} \mathrm{H}_{13} \mathrm{~F}_{5} \mathrm{O}_{1} \mathrm{~N}[\mathrm{M}+\mathrm{H}]^{+}:$342.0912, found: 342.0921 .

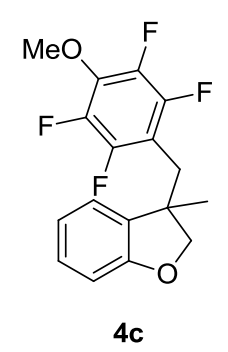

3-methyl-3-(2,3,5,6-tetrafluoro-4-methoxybenzyl)-2,3-dihydrobenzofuran (4c): $71 \mathrm{mg}$;

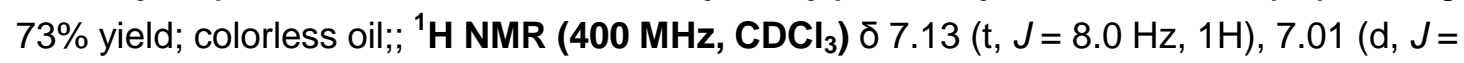
$7.2 \mathrm{~Hz}, 1 \mathrm{H}), 6.86(\mathrm{t}, J=7.2 \mathrm{~Hz}, 1 \mathrm{H}), 6.76(\mathrm{~d}, J=8.0 \mathrm{~Hz}, 1 \mathrm{H}), 4.49(\mathrm{~d}, J=8.8 \mathrm{~Hz}, 1 \mathrm{H}), 4.09$ $(\mathrm{d}, J=8.8 \mathrm{~Hz}, 1 \mathrm{H}), 4.04(\mathrm{~s}, 3 \mathrm{H}), 3.02-2.93(\mathrm{~m}, 2 \mathrm{H}), 1.37(\mathrm{~s}, 3 \mathrm{H}) ;{ }^{13} \mathrm{C}$ NMR (100 MHz, $\left.\mathrm{CDCl}_{3}\right) \delta 159.3,145.5\left(\mathrm{dm}, J_{\mathrm{F}}=242.5 \mathrm{~Hz}\right), 140.6\left(\mathrm{dm}, J_{\mathrm{F}}=245.0 \mathrm{~Hz}\right), 138.2\left(\mathrm{dm}, J_{\mathrm{F}}=\right.$ 247.0 Hz), 133.5, 128.6, 122.8, 120.6, 109.5 (t, $J=20.0 \mathrm{~Hz}$ ), 82.0 (d, $J=2.0 \mathrm{~Hz}$ ), 62.0, 46.6, 32.5, 23.7; ${ }^{19} \mathbf{F}$ NMR (376 MHz, $\mathbf{C D C l}_{3}$ ) $\delta-142.1$ (m, 2F), -158.3 (m, 2F); IR (neat, $\left.\mathbf{c m}^{-1}\right):$ 2966, 1654, 1597, 1492, 1417, 1231, 1131, 1016, 986, 753; HRMS (ESI) calcd for $\mathrm{C}_{17} \mathrm{H}_{15} \mathrm{~F}_{4} \mathrm{O}_{2}[\mathrm{M}+\mathrm{H}]^{+}:$327.1003, found: 327.0997. 


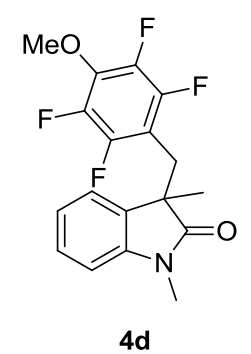

1,3-dimethyl-3-(2,3,5,6-tetrafluoro-4-methoxybenzyl)indolin-2-one (4d): $82 \mathrm{mg} ; 78 \%$ yield; colorless oil; ${ }^{1} \mathbf{H}$ NMR (400 MHz, $\left.\mathbf{C D C l}_{3}\right) \delta$ 7.25-7.22 (m, $\left.1 \mathrm{H}\right), 7.05-6.98(\mathrm{~m}, 2 \mathrm{H})$, $6.80(\mathrm{~d}, J=7.6 \mathrm{~Hz}, 1 \mathrm{H}), 4.02(\mathrm{~s}, 3 \mathrm{H}), 3.20(\mathrm{~s}, 3 \mathrm{H}), 3.10(\mathrm{~s}, 2 \mathrm{H}), 1.44(\mathrm{~s}, 3 \mathrm{H}) ;{ }^{13} \mathrm{C}$ NMR $\left(\mathbf{1 0 0} \mathbf{M H z}, \mathrm{CDCl}_{3}\right) \delta 179.0,145.4\left(\mathrm{dm}, J_{\mathrm{F}}=241.5 \mathrm{~Hz}\right), 142.7,140.4\left(\mathrm{dm}, J_{\mathrm{F}}=245.0 \mathrm{~Hz}\right)$, $138.1\left(\mathrm{dm}, J_{F}=215.0 \mathrm{~Hz}\right), 132.5,128.1,122.8,122.2,108.7-107.8(\mathrm{~m}), 61.8(\mathrm{~d}, J=3.0$ $\mathrm{Hz}$ ), 47.7, 30.6, 26.0, 21.8; ${ }^{19} \mathrm{~F}$ NMR (376 MHz, $\mathrm{CDCl}_{3}$ ) $\delta-141.3(\mathrm{~m}, 2 \mathrm{~F}),-158.7(\mathrm{~m}, 2 \mathrm{~F})$; IR (neat, $\mathbf{~ c m}^{-1}$ ): 2935, 1719, 1613, 1493, 1378, 1350, 1126, 1012, 984, 928, 756; HRMS (ESI) calcd for $\mathrm{C}_{18} \mathrm{H}_{16} \mathrm{~F}_{4} \mathrm{O}_{2} \mathrm{~N}[\mathrm{M}+\mathrm{H}]^{+}:$354.1112, found: 354.1107 .<smiles>CC(=O)c1cc(F)c(F)c(CC2(C)COc3ccccc32)c1F</smiles>

1-(2,4,5-trifluoro-3-((3-methyl-2,3-dihydrobenzofuran-3-yl)methyl)phenyl)ethanone (4e): $55 \mathrm{mg}$; $58 \%$ yield; colorless oil; ${ }^{1} \mathrm{H}$ NMR (400 MHz, $\left.\mathrm{CDCl}_{3}\right)$ ठ 7.63-7.56 (m, $\left.1 \mathrm{H}\right), 7.14$ $(\mathrm{t}, J=7.2 \mathrm{~Hz}, 1 \mathrm{H}), 6.95(\mathrm{~d}, J=7.6 \mathrm{~Hz}, 1 \mathrm{H}), 6.85(\mathrm{t}, J=7.2 \mathrm{~Hz}, 1 \mathrm{H}), 6.76(\mathrm{~d}, J=8.0 \mathrm{~Hz}$, $1 \mathrm{H}), 4.54(\mathrm{~d}, J=8.8 \mathrm{~Hz}, 1 \mathrm{H}), 4.13(\mathrm{~d}, J=9.2 \mathrm{~Hz}, 1 \mathrm{H}), 3.05(\mathrm{dd}, J=34.4 \mathrm{~Hz}, J=13.2 \mathrm{~Hz}$, 2H), 2.50 (d, $J=5.6 \mathrm{~Hz}, 3 \mathrm{H}), 1.43(\mathrm{~s}, 3 \mathrm{H}) ;{ }^{13} \mathrm{C} \mathrm{NMR}\left(100 \mathrm{MHz}, \mathrm{CDCl}_{3}\right) \delta 193.5,159.3$, $156.7\left(\mathrm{dm}, J_{F}=252.0 \mathrm{~Hz}\right), 152.2\left(\mathrm{dm}, J_{F}=255.0 \mathrm{~Hz}\right), 146.8\left(\mathrm{dm}, J_{F}=248.0 \mathrm{~Hz}\right), 133.1$, 128.7, 122.9, 121.4, 121.2, 120.5, 117.1-116.7 (m), 115.9 (d, $J=20.0 \mathrm{~Hz}), 109.8,82.3$, 46.7, 33.2, $31.2(\mathrm{~d}, J=8.0 \mathrm{~Hz}), 23.5 ;{ }^{19} \mathrm{~F}$ NMR (376 MHz, $\left.\mathrm{CDCl}_{3}\right) \delta-111.3(\mathrm{~m}, 1 \mathrm{~F}),-126.3$ (m, 1F), -140.4 (m, 1F); IR (neat, $\left.\mathbf{c m}^{-1}\right)$ : 2967, 1690, 1612, 1482, 1365, 1257, 1200, 986, 812, 754; HRMS (ESI) calcd for $\mathrm{C}_{18} \mathrm{H}_{16} \mathrm{~F}_{3} \mathrm{O}_{2}[\mathrm{M}+\mathrm{H}]^{+}:$:321.1097, found: 321.1103.

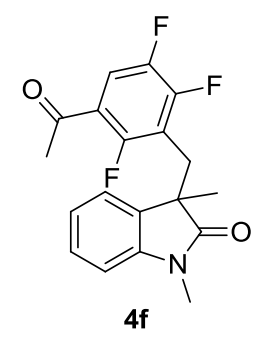

3-(3-acetyl-2,5,6-trifluorobenzyl)-1,3-dimethylindolin-2-one (4f): $67 \mathrm{mg} ; 65 \%$ yield; colorless oil; ${ }^{1} \mathbf{H}$ NMR $\left(400 ~ M H z, \mathbf{C D C l}_{3}\right)$ ठ 7.59-7.52 (m, 1H), 7.27-7.23 (m, 1H), 7.06-6.99 $(\mathrm{m}, 2 \mathrm{H}), 6.81(\mathrm{~d}, J=8.0 \mathrm{~Hz}, 1 \mathrm{H}), 3.21(\mathrm{~s}, 3 \mathrm{H}), 3.19(\mathrm{~s}, 2 \mathrm{H}), 2.53(\mathrm{~d}, J=5.6 \mathrm{~Hz}, 3 \mathrm{H}), 1.48$ (s, 3H); ${ }^{13} \mathrm{C}$ NMR $\left(100 \mathrm{MHz}, \mathrm{CDCl}_{3}\right) \delta$ 193.3, 178.9, $156.5\left(\mathrm{dm}, \mathrm{J}_{\mathrm{F}}=252.0 \mathrm{~Hz}\right), 152.0(\mathrm{dm}$, 
$\left.J_{F}=256.0 \mathrm{~Hz}\right), 146.6\left(\mathrm{dm}, J_{F}=245.0 \mathrm{~Hz}\right), 142.6,132.2,122.9,122.1,121.2-121.0(\mathrm{~m})$, 116.1-115.6 (m), 107.9, 47.8, 31.1(t, $J=7.0 \mathrm{~Hz}), 26.1,21.9 ;{ }^{19} \mathrm{~F}$ NMR (376 MHz, $\left.\mathbf{C D C l}_{3}\right)$ ס-110.4 (m, 1F), -125.2 (m, 1F), -140.6 (m, 1F); IR (neat, $\left.\mathbf{c m}^{-1}\right)$ : 2932, 1711, 1612, 1491, 1378, 1257, 1201, 1104, 1035, 755; HRMS (ESI) calcd for $\mathrm{C}_{19} \mathrm{H}_{17} \mathrm{~F}_{3} \mathrm{NO}_{2}[\mathrm{M}+\mathrm{H}]^{+}: 348.1206$, found: 348.1208 .

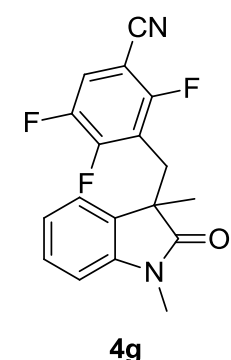

3-((1,3-dimethyl-2-oxoindolin-3-yl)methyl)-2,4,5-trifluorobenzonitrile (4g): $87 \mathrm{mg}$; 88\% yield; yellow solid; $\mathrm{mp}=96-98{ }^{\circ} \mathrm{C} ;{ }^{1} \mathbf{H}$ NMR $\left(400 \mathrm{MHz}, \mathrm{CDCl}_{3}\right) \delta 7.36-7.25(\mathrm{~m}, 2 \mathrm{H})$, 7.12-7.02 (m, 2H), $6.83(\mathrm{~d}, J=7.6 \mathrm{~Hz}, 1 \mathrm{H}), 3.23-3.11(\mathrm{~m}, 5 \mathrm{H}), 1.46(\mathrm{~s}, 3 \mathrm{H}) ;{ }^{13} \mathrm{C}$ NMR $(100$ $\left.\mathbf{M H z}, \mathbf{C D C l}_{3}\right) \delta 178.3,158.2\left(\mathrm{dm}, J_{\mathrm{F}}=257.0 \mathrm{~Hz}\right), 152.5\left(\mathrm{dm}, J_{\mathrm{F}}=259.0 \mathrm{~Hz}\right), 146.4\left(\mathrm{dm}, J_{\mathrm{F}}\right.$ $=249.0 \mathrm{~Hz}), 142.5,131.9,128.4,122.6,122.3,118.9,118.7,117.1-116.7(\mathrm{~m}), 112.2$,

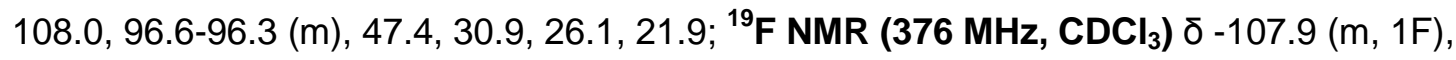
-122.3 (m, 1F), -138.4 (m, 1F); IR (neat, cm$^{-1}$ ): 3059, 1718, 1613, 1493, 1354, 1255, 1120 , 755; HRMS (ESI) calcd for $\mathrm{C}_{18} \mathrm{H}_{14} \mathrm{~F}_{3} \mathrm{~N}_{2} \mathrm{O}_{1}[\mathrm{M}+\mathrm{H}]^{+}: 331.1053$, found: 331.1047 .

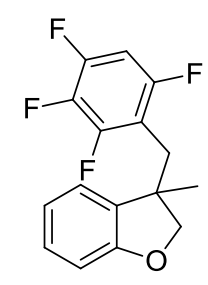

4h

3-methyl-3-(2,3,4,6-tetrafluorobenzyl)-2,3-dihydrobenzofuran (4h): $46 \mathrm{mg}$; $52 \%$ yield; colorless oil; ${ }^{1} \mathrm{H}$ NMR $\left(400 \mathrm{MHz}, \mathrm{CDCl}_{3}\right) \delta 7.14(\mathrm{t}, J=7.2 \mathrm{~Hz}, 1 \mathrm{H}), 6.99(\mathrm{~d}, J=6.8 \mathrm{~Hz}, 1 \mathrm{H})$, $6.86(\mathrm{t}, J=7.2 \mathrm{~Hz}, 1 \mathrm{H}), 6.78-6.69(\mathrm{~m}, 2 \mathrm{H}), 4.50(\mathrm{~d}, J=8.8 \mathrm{~Hz}, 1 \mathrm{H}), 4.09(\mathrm{~d}, J=8.8 \mathrm{~Hz}$, $1 \mathrm{H}), 2.96(\mathrm{q}, J=13.6 \mathrm{~Hz}, 2 \mathrm{H}), 1.38(\mathrm{~s}, 3 \mathrm{H}) ;{ }^{13} \mathbf{C ~ N M R}\left(100 \mathrm{MHz}, \mathbf{C D C l}_{3}\right) \delta 159.3,155.7$ $\left(\mathrm{dm}, J_{F}=243.0 \mathrm{~Hz}\right), 150.2\left(\mathrm{dm}, J_{F}=244.0 \mathrm{~Hz}\right), 149.3\left(\mathrm{dm}, J_{F}=245.5 \mathrm{~Hz}\right), 137.0\left(\mathrm{dm}, J_{F}=\right.$ $250.0 \mathrm{~Hz}), 133.5,128.6,122.9,120.6,111.3\left(\mathrm{t}, J_{\mathrm{F}}=20.0 \mathrm{~Hz}\right), 109.8,100.7-100.1(\mathrm{~m})$, 82.2, 46.6, 32.6, 23.7; ${ }^{19} \mathrm{~F}$ NMR (376 MHz, $\left.\mathbf{C D C l}_{3}\right) \delta-115.6$ (d, $\left.J_{\mathrm{F}}=10.5 \mathrm{~Hz}, 1 \mathrm{~F}\right),-132.9$ $(\mathrm{m}, 1 \mathrm{~F}),-134.0(\mathrm{~m}, 1 \mathrm{~F}),-165.0(\mathrm{~m}, 1 \mathrm{~F})$; IR (neat, $\left.\mathbf{c m}^{-1}\right):$ 2968, 1648, 1517, 1467, 1255, 1113, 1035, 980, 831, 753; HRMS (ESI) calcd for $\mathrm{C}_{16} \mathrm{H}_{13} \mathrm{~F}_{4} \mathrm{O}_{1}[\mathrm{M}+\mathrm{H}]^{+}: 297.0897$, found: 297.0896 . 


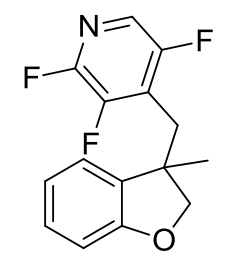

$4 \mathbf{i}$

2,3,5-trifluoro-4-((3-methyl-2,3-dihydrobenzofuran-3-yl)methyl)pyridine (4i): 53 mg; 64\% yield; yellow solid; mp $=76-78{ }^{\circ} \mathrm{C} ;{ }^{1} \mathbf{H}$ NMR $\left(400 \mathbf{M H z}, \mathbf{C D C l}_{3}\right) \delta 7.78(\mathrm{~s}, 1 \mathrm{H})$, 7.17-7.13 (m, 1H), $6.99(\mathrm{~d}, J=7.2 \mathrm{~Hz}, 1 \mathrm{H}), 6.88(\mathrm{t}, J=7.2 \mathrm{~Hz}, 1 \mathrm{H}), 6.76(\mathrm{~d}, J=8.0 \mathrm{~Hz}$, $1 \mathrm{H}), 4.53(\mathrm{~d}, J=8.8 \mathrm{~Hz}, 1 \mathrm{H}), 4.11(\mathrm{~d}, J=9.2 \mathrm{~Hz}, 1 \mathrm{H}), 3.07(\mathrm{q}, J=13.6 \mathrm{~Hz}, 2 \mathrm{H}), 1.43(\mathrm{~s}$, 3H); ${ }^{13} \mathrm{C}$ NMR $\left(100 \mathrm{MHz}, \mathrm{CDCl}_{3}\right) \delta 159.2,156.9\left(\mathrm{~d}, \mathrm{~J}_{\mathrm{F}}=3.0 \mathrm{~Hz}\right), 154.4\left(\mathrm{~d}, J_{\mathrm{F}}=4.0 \mathrm{~Hz}\right)$, $148.1\left(\mathrm{dd}, J_{\mathrm{F}}=234.0 \mathrm{~Hz}, J_{\mathrm{F}}=15.0 \mathrm{~Hz}\right), 143.7\left(\mathrm{dm}, J_{\mathrm{F}}=261.0 \mathrm{~Hz}\right), 132.7,128.9$, 128.0-127.5 (m), 127.2-126.8 (m), 122.7, 120.7, 109.9, $82.0\left(\mathrm{t}, J_{F}=3.0 \mathrm{~Hz}\right), 46.7,33.3$, 23.7; ${ }^{19} \mathrm{~F}$ NMR (376 MHz, $\left.\mathrm{CDCl}_{3}\right) \delta-89.8\left(\mathrm{t}, J_{\mathrm{F}}=27.4 \mathrm{~Hz}, 1 \mathrm{~F}\right),-129.9\left(\mathrm{~d}, J_{\mathrm{F}}=28.2 \mathrm{~Hz}, 1 \mathrm{~F}\right)$, $-137.6\left(t, J_{F}=25.9 \mathrm{~Hz}, 1 \mathrm{~F}\right)$; IR (neat, $\left.\mathbf{c m}^{-1}\right)$ : 2969, 1597, 1479, 1265, 1058, 984, 831, 753; HRMS (ESI) calcd for $\mathrm{C}_{15} \mathrm{H}_{13} \mathrm{~F}_{3} \mathrm{NO}_{1}[\mathrm{M}+\mathrm{H}]^{+}: 280.0944$, found: 280.0956 .<smiles>CN1C(=O)C(C)(Cc2c(F)cnc(F)c2F)c2ccccc21</smiles>

1,3-dimethyl-3-((2,3,5-trifluoropyridin-4-yl)methyl)indolin-2-one (4j): $74 \mathrm{mg} ; 81 \%$ yield; yellow solid; $\mathrm{mp}=142-146{ }^{\circ} \mathrm{C} ;{ }^{1} \mathbf{H}$ NMR $\left(400 \mathbf{~ M H z}, \mathbf{C D C l}_{3}\right) \delta 7.74(\mathrm{~s}, 1 \mathrm{H}), 7.29-7.24$ $(\mathrm{m}, 1 \mathrm{H}), 7.10-7.01(\mathrm{~m}, 2 \mathrm{H}), 6.81(\mathrm{~d}, J=8.0 \mathrm{~Hz}, 1 \mathrm{H}), 3.25-3.17(\mathrm{~m}, 5 \mathrm{H}), 1.48(\mathrm{~s}, 3 \mathrm{H}) ;{ }^{13} \mathrm{C}$ NMR $\left(100 \mathrm{MHz}, \mathrm{CDCl}_{3}\right) \delta 178.5,156.8\left(\mathrm{~d}, J_{\mathrm{F}}=3.0 \mathrm{~Hz}\right), 154.2\left(\mathrm{~d}, J_{\mathrm{F}}=4.0 \mathrm{~Hz}\right), 147.9$ (dd, $\left.J_{F}=235.0 \mathrm{~Hz}, J_{F}=16.0 \mathrm{~Hz}\right), 142.6,142.4\left(\mathrm{dm}, J_{F}=257.5 \mathrm{~Hz}\right), 131.8,128.5,127.8-127.3$ (m), 126.2-125.8 (m), 122.8, 122.4, 108.1, 47.6, 31.1, 26.2, 22.3; ${ }^{19} \mathrm{~F}$ NMR (376 MHz, $\left.\mathrm{CDCl}_{3}\right) \delta-89.9\left(\mathrm{t}, J_{\mathrm{F}}=27.1 \mathrm{~Hz}, 1 \mathrm{~F}\right),-129.0\left(\mathrm{~d}, J_{\mathrm{F}}=28.6 \mathrm{~Hz}, 1 \mathrm{~F}\right),-136.6\left(\mathrm{t}, J_{\mathrm{F}}=25.6 \mathrm{~Hz}\right.$, 1F); IR (neat, $\mathbf{c m}^{-1}$ ): 2971, 1714, 1613, 1476, 1381, 1262, 1212, 1104, 1029, 905, 770; HRMS (ESI) calcd for $\mathrm{C}_{16} \mathrm{H}_{14} \mathrm{~F}_{3} \mathrm{~N}_{2} \mathrm{O}_{1}[\mathrm{M}+\mathrm{H}]^{+}: 307.1053$, found: 307.1050 .<smiles>CN1C(=O)C(C)(Cc2c(F)c(F)nc(F)c2F)c2ccccc21</smiles>

1,3-dimethyl-3-((perfluoropyridin-4-yl)methyl)indolin-2-one (4k): $93 \mathrm{mg} ; 96 \%$ yield; yellow solid; $\mathrm{mp}=157-159^{\circ} \mathrm{C}$; ${ }^{1} \mathrm{H}$ NMR $\left(400 \mathrm{MHz}, \mathrm{CDCl}_{3}\right) \delta 7.28(\mathrm{t}, \mathrm{J}=7.6 \mathrm{~Hz}, 1 \mathrm{H}), 7.14$ (d, $J=7.2 \mathrm{~Hz}, 1 \mathrm{H}), 7.05$ (t, $J=7.6 \mathrm{~Hz}, 1 \mathrm{H}), 6.83(\mathrm{~d}, J=7.6 \mathrm{~Hz}, 1 \mathrm{H}), 3.31-3.19(\mathrm{~m}, 5 \mathrm{H})$, $1.49(\mathrm{~s}, 3 \mathrm{H}) ;{ }^{13} \mathrm{C}$ NMR $\left(100 \mathrm{MHz}, \mathrm{CDCl}_{3}\right) \delta 178.2,143.1\left(\mathrm{dm}, J_{\mathrm{F}}=240.0 \mathrm{~Hz}\right), 142.6,140.6$ 
$\left(\mathrm{dm}, J_{\mathrm{F}}=255.0 \mathrm{~Hz}\right), 131.6,130.2-129.8(\mathrm{~m}), 128.6,122.7,122.6,108.2,47.5,31.7,26.2$, 22.4; ${ }^{19} \mathrm{~F}$ NMR (376 MHz, $\mathbf{C D C l}_{3}$ ) $\delta-91.6(\mathrm{~m}, 2 \mathrm{~F}),-141.0(\mathrm{~m}, 2 \mathrm{~F})$; IR (neat, $\mathbf{c m}^{-1}$ ): 2971, 1717, 1612, 1463, 1381, 1252, 1124, 905, 768; HRMS (ESI) calcd for $\mathrm{C}_{16} \mathrm{H}_{13} \mathrm{~F}_{4} \mathrm{~N}_{2} \mathrm{O}_{1}[\mathrm{M}+\mathrm{H}]^{+}:$: 325.0959, found: 325.0963 .

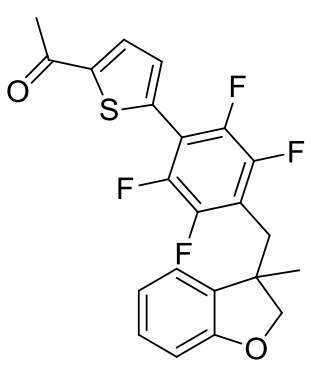

$3 a^{\prime}$

1-(5-(2,3,5,6-tetrafluoro-4-((3-methyl-2,3-dihydrobenzofuran-3-yl)methyl)phenyl)thio phen-2-yl)ethanone (3a'): $50 \mathrm{mg}$; $40 \%$ yield; yellow solid; $\mathrm{mp}=98-100{ }^{\circ} \mathrm{C}$; ${ }^{1} \mathrm{H}$ NMR (400 $\left.\mathbf{M H z}, \mathrm{CDCl}_{3}\right) \delta 7.72(\mathrm{~d}, J=3.6 \mathrm{~Hz}, 1 \mathrm{H}), 7.58(\mathrm{~d}, J=4.0 \mathrm{~Hz}, 1 \mathrm{H}), 7.16(\mathrm{t}, J=7.6 \mathrm{~Hz}, 1 \mathrm{H})$, $7.05(\mathrm{~d}, J=7.6 \mathrm{~Hz}, 1 \mathrm{H}), 6.90(\mathrm{t}, J=7.6 \mathrm{~Hz}, 1 \mathrm{H}), 6.79(\mathrm{~d}, J=8.0 \mathrm{~Hz}, 1 \mathrm{H}), 4.54(\mathrm{~d}, J=9.2$ $\mathrm{Hz}, 1 \mathrm{H}), 4.14(\mathrm{~d}, J=8.8 \mathrm{~Hz}, 1 \mathrm{H}), 3.08(\mathrm{q}, J=13.2 \mathrm{~Hz}, 2 \mathrm{H}), 2.61(\mathrm{~s}, 3 \mathrm{H}), 1.44(\mathrm{~s}, 3 \mathrm{H}) ;{ }^{13} \mathrm{C}$ NMR $\left(100 \mathrm{MHz}, \mathrm{CDCl}_{3}\right) \delta 190.7,159.2,145.7\left(\mathrm{dm}, J_{\mathrm{F}}=221.0 \mathrm{~Hz}\right), 145.4,143.4\left(\mathrm{dm}, J_{\mathrm{F}}=\right.$ $242.0 \mathrm{~Hz}), 135.6,133.2,131.9,130.7\left(\mathrm{t}, J_{\mathrm{F}}=6.0 \mathrm{~Hz}\right), 128.8,122.8,120.7,116.6\left(\mathrm{t}, J_{\mathrm{F}}=\right.$ $19.0 \mathrm{~Hz}), 111.9\left(\mathrm{~d}, J_{\mathrm{F}}=15.0 \mathrm{~Hz}\right), 109.9,82.1,46.8,33.3,26.9,23.8 ;{ }^{19} \mathrm{~F}$ NMR (376 MHz, $\mathbf{C D C l}_{3}$ ) $\delta-139.7$ (m, 2F), -140.4 (m, 2F); IR (neat, $\mathbf{c m}^{-1}$ ): 2967, 1667, 1479, 1442, 1270, 1153, 1017, 984, 753; HRMS (ESI) calcd for C22H17F4O2S[M+H] ${ }^{+}: 421.0880$, found: 421.0883 .

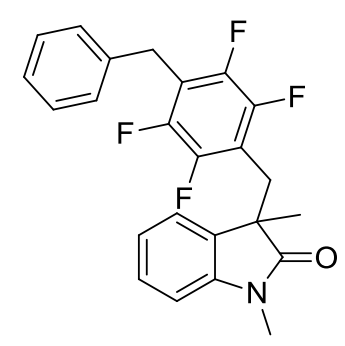

$3 \mathbf{j}^{\prime}$

3-(4-benzyl-2,3,5,6-tetrafluorobenzyl)-1,3-dimethylindolin-2-one (3j'): $120 \mathrm{mg}$; 97\% yield; colorless oil; ${ }^{1} \mathbf{H}$ NMR (400 MHz, $\left.\mathbf{C D C l}_{3}\right) \delta$ 7.28-7.18 (m, 6H), 7.02-6.96 (m, 2H), $6.77(\mathrm{~d}, J=7.6 \mathrm{~Hz}, 1 \mathrm{H}), 3.98(\mathrm{~s}, 2 \mathrm{H}), 3.19-3.12(\mathrm{~m}, 5 \mathrm{H}), 1.44(\mathrm{~s}, 3 \mathrm{H}) ;{ }^{13} \mathrm{C}$ NMR (100 MHz, $\left.\mathrm{CDCl}_{3}\right) \delta 179.0,146.3\left(\mathrm{dm}, J_{\mathrm{F}}=246.0 \mathrm{~Hz}\right), 144.2\left(\mathrm{dm}, J_{\mathrm{F}}=209.0 \mathrm{~Hz}\right), 142.6,137.6,132.4$, 128.6, 128.2, 128.1, 126.6, 122.9, 122.2, $118.1\left(\mathrm{t}, J_{F}=18.5 \mathrm{~Hz}\right), 113.5\left(\mathrm{t}, J_{F}=18.5 \mathrm{~Hz}\right)$, 107.9, 47.8, 31.0, 28.4, 26.1, 21.9; ${ }^{19} \mathbf{F}$ NMR (376 MHz, $\left.\mathbf{C D C l}_{3}\right) \delta-140.5(\mathrm{~m}, 2 \mathrm{~F}),-144.5$ (m, 2F); IR (neat, $\mathbf{c m}^{-1}$ ): 2935, 1717, 1613, 1486, 1377, 1350, 1273, 1120, 1036, 990, 753; HRMS (ESI) calcd for $\mathrm{C}_{24} \mathrm{H}_{20} \mathrm{~F}_{4} \mathrm{O}_{1} \mathrm{~N}[\mathrm{M}+\mathrm{H}]^{+}: 414.1476$, found: 414.1477 . 
<smiles>CC(C)(C)OC/C=C/c1c(F)c(F)c(CC2(C)C(=O)Nc3ccccc32)c(F)c1F</smiles>

$3 \mathbf{k}^{\prime}$

(E)-tert-butyl

3-(4-((1-benzyl-3-methyl-2-oxoindolin-3-yl)methyl)-2,3,5,6-tetrafluorophenyl)acrylate (3k'): 115 mg; 73\% yield; colorless oil; ${ }^{1} \mathrm{H}$ NMR (400 MHz, $\left.\mathbf{C D C l}_{3}\right) \delta 7.56(\mathrm{~d}, \mathrm{~J}=16.4 \mathrm{~Hz}$, $1 \mathrm{H}), 7.30-7.20(\mathrm{~m}, 5 \mathrm{H}), 7.13(\mathrm{t}, J=7.6 \mathrm{~Hz}, 2 \mathrm{H}), 6.99(\mathrm{t}, J=7.6 \mathrm{~Hz}, 2 \mathrm{H}), 6.70-6.63(\mathrm{~m}, 2 \mathrm{H})$, $5.01(\mathrm{~d}, J=7.8 \mathrm{~Hz}, 1 \mathrm{H}), 4.75(\mathrm{~d}, J=7.8 \mathrm{~Hz}, 1 \mathrm{H}), 3.25(\mathrm{dd}, J=38.8 \mathrm{~Hz}, J=14.0 \mathrm{~Hz}, 2 \mathrm{H})$, $1.54(\mathrm{~s}, 12 \mathrm{H}) ;{ }^{13} \mathrm{C}$ NMR $\left(100 \mathrm{MHz}, \mathrm{CDCl}_{3}\right) \delta 178.7,165.4,144.9\left(\mathrm{dm}, J_{\mathrm{F}}=249.5 \mathrm{~Hz}\right)$, $144.7\left(\mathrm{dm}, J_{F}=250.5 \mathrm{~Hz}\right), 141.8,135.7,132.2,128.7-127.1(\mathrm{~m}), 123.0,122.4,116.6\left(\mathrm{t}, J_{F}\right.$ $=18.5 \mathrm{~Hz}), 113.2\left(\mathrm{t}, J_{\mathrm{F}}=13.0 \mathrm{~Hz}\right), 109.1,81.2,48.0,43.7,31.2,28.0,22.9 ;{ }^{19} \mathrm{~F}$ NMR (376 MHz, $\mathbf{C D C l}_{3}$ ) $\delta-139.8$ (m, 2F), -141.1 (m, 2F); IR (neat, $\mathbf{c m}^{-1}$ ): 2977, 2933, 1717, 1612, 1486, 1361, 1268, 1155, 1007, 850, 745; HRMS (ESI) calcd for $\mathrm{C}_{30} \mathrm{H}_{28} \mathrm{~F}_{4} \mathrm{NO}_{3}[\mathrm{M}+\mathrm{H}]^{+}$: 526.2000, found: 526.1995 .

\section{References.}

(1) (a) Newman, S. G.; Lautens, M. J. Am. Chem. Soc. 2011, 133, 1778. (b) Gao, Y.; Xiong, W.; Chen, H.; Wu, W.; Peng, J.; Gao, Y.; Jiang, H. J. Org. Chem. 2015, 80, 7456.

(2) (a) Pinto, A.; Jia, Y.-X.; Neuville, L.; Zhu, J.-P. Chem. Eur. J. 2007, 13, 961. (b) Ishikura, M.; Takahashi, N.; Yamada, K.; Yanada, R. Tetrahedron. 2006, 62, 11580. (c) Liu, X.; Ma, X.; Huang, Y.; Gu, Z. Org. Lett. 2013, 15, 4814. 


\section{Crystallographic Data of $3 k$ and $4 a$.}

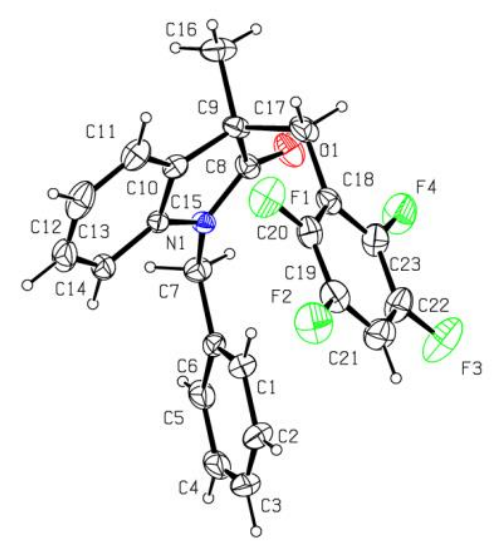

\section{Structure of 3k CCDC: 1448627}

\section{Datablock:}

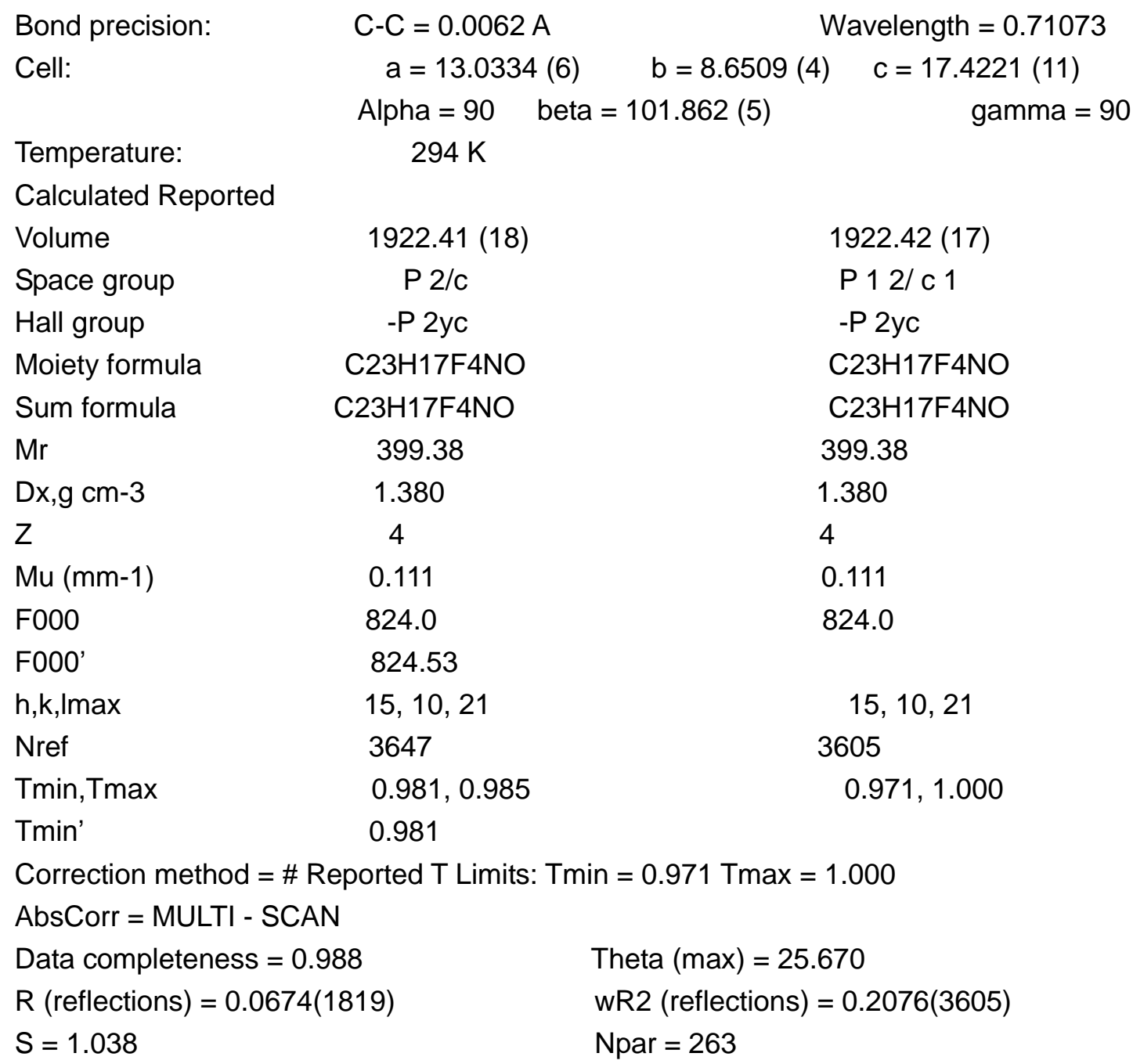




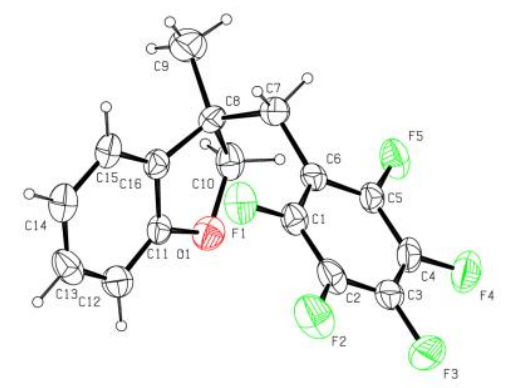

Structure of $4 a \quad$ CCDC: 1448626

\section{Datablock:}

\begin{tabular}{|c|c|c|}
\hline Bond precision: & $C-C=0.0085 \mathrm{~A}$ & Wavelength $=0.71000$ \\
\hline \multirow[t]{2}{*}{ Cell: } & $a=22.905$ & $c=15.6010(17)$ \\
\hline & Alpha $=90$ & gamma $=90$ \\
\hline \multirow[t]{2}{*}{ Temperature: } & $293 \mathrm{~K}$ & \\
\hline & Calculated & Reported \\
\hline Volume & $2796.7(7)$ & $2796.7(7)$ \\
\hline Space group & $\mathrm{pbca}$ & \\
\hline Hall group & $-P 2 a c \quad 2 a b$ & \\
\hline Moiety formula & $\mathrm{C} 16 \mathrm{H} 11 \mathrm{~F} 5 \mathrm{O}$ & \\
\hline Sum formula & $\mathrm{C} 16 \mathrm{H} 11 \mathrm{~F} 5 \mathrm{O}$ & $\mathrm{C} 32 \mathrm{H} 22 \mathrm{~F} 10 \mathrm{O} 2$ \\
\hline $\mathrm{Mr}$ & 314.25 & 628.50 \\
\hline$D x, g \mathrm{~cm}-3$ & 1.493 & 1.493 \\
\hline Z & 8 & 4 \\
\hline $\mathrm{Mu}(\mathrm{mm}-1)$ & 0.135 & 0.138 \\
\hline F000 & 1280.0 & 1280.0 \\
\hline F000' & 1281.05 & \\
\hline $\mathrm{h}, \mathrm{k}, \mathrm{Imax}$ & $28,9,19$ & $28,9,19$ \\
\hline Nref & 2757 & 2752 \\
\hline \multicolumn{3}{|l|}{ Tmin,Tmax } \\
\hline \multicolumn{3}{|l|}{ Tmin' } \\
\hline \multicolumn{3}{|c|}{ Correction method $=$ Not given } \\
\hline \multicolumn{2}{|c|}{ Data completeness $=0.998$} & Theta $(\max )=25.980$ \\
\hline \multicolumn{2}{|c|}{$\mathrm{R}$ (reflections) $=0.0835(998)$} & wR2 $($ reflections $)=0.2986(2752)$ \\
\hline \multicolumn{3}{|c|}{$S=0.993 \quad N p a r=200$} \\
\hline
\end{tabular}




\section{Copies of ${ }^{1} \mathrm{H},{ }^{19} \mathrm{~F}$ and ${ }^{13} \mathrm{C}$ Spectra.}
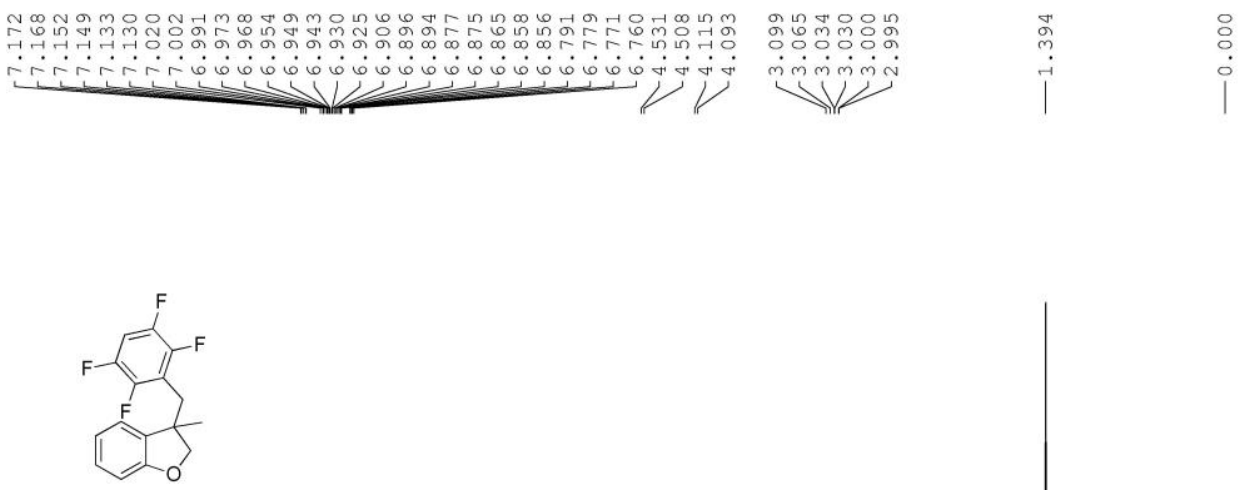

$3 a$
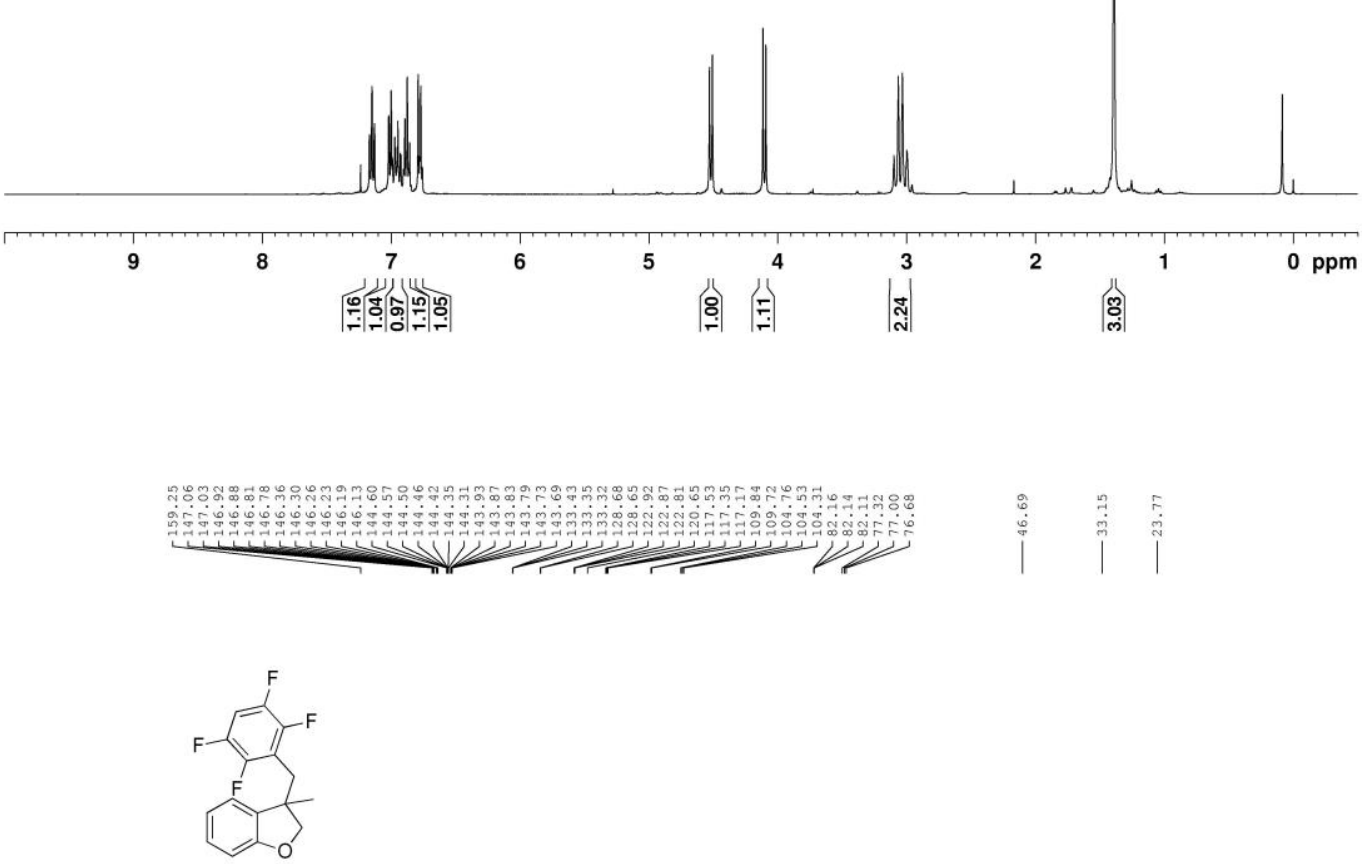

3a

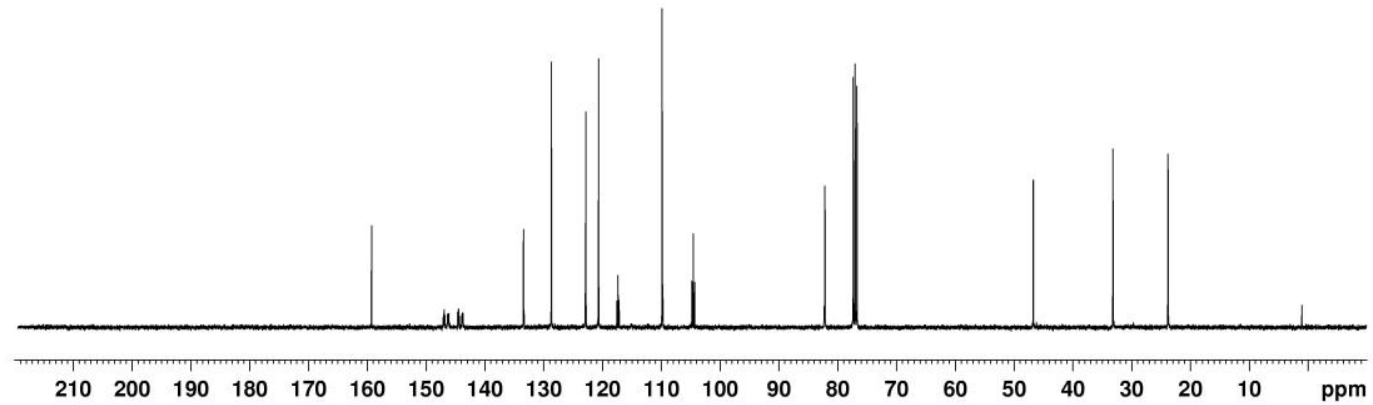




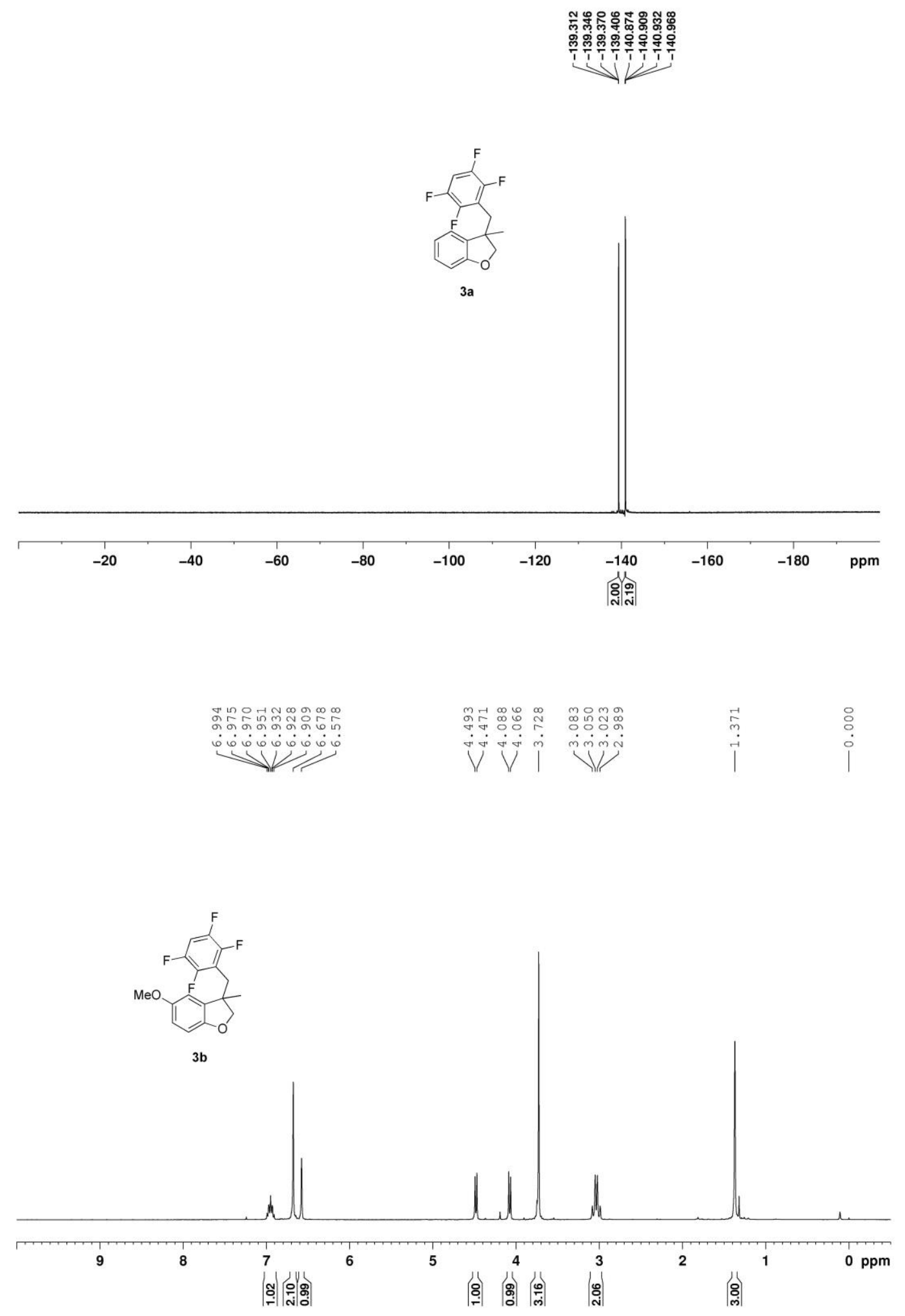




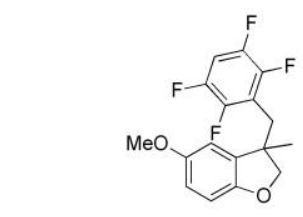

3b

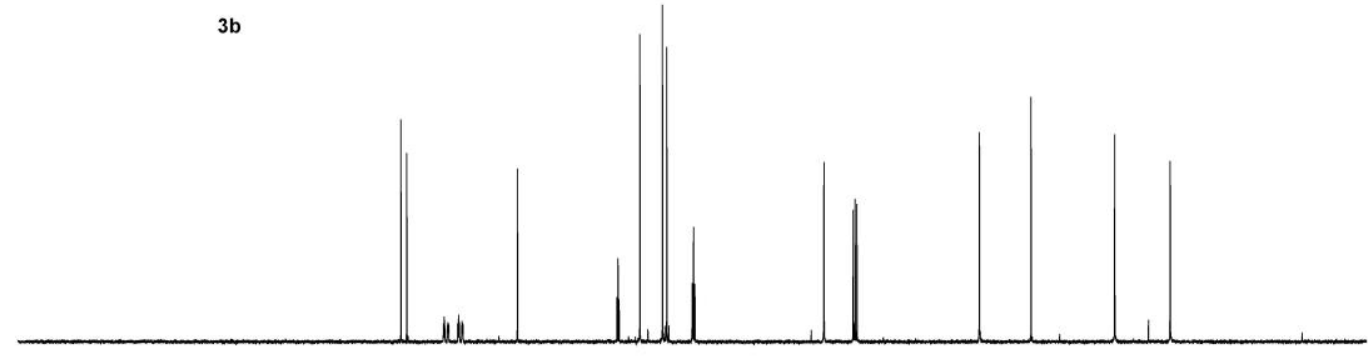

$\begin{array}{llllllllllllllllllllll}210 & 200 & 190 & 180 & 170 & 160 & 150 & 140 & 130 & 120 & 110 & 100 & 90 & 80 & 70 & 60 & 50 & 40 & 30 & 20 & 10 & \mathrm{ppm}\end{array}$

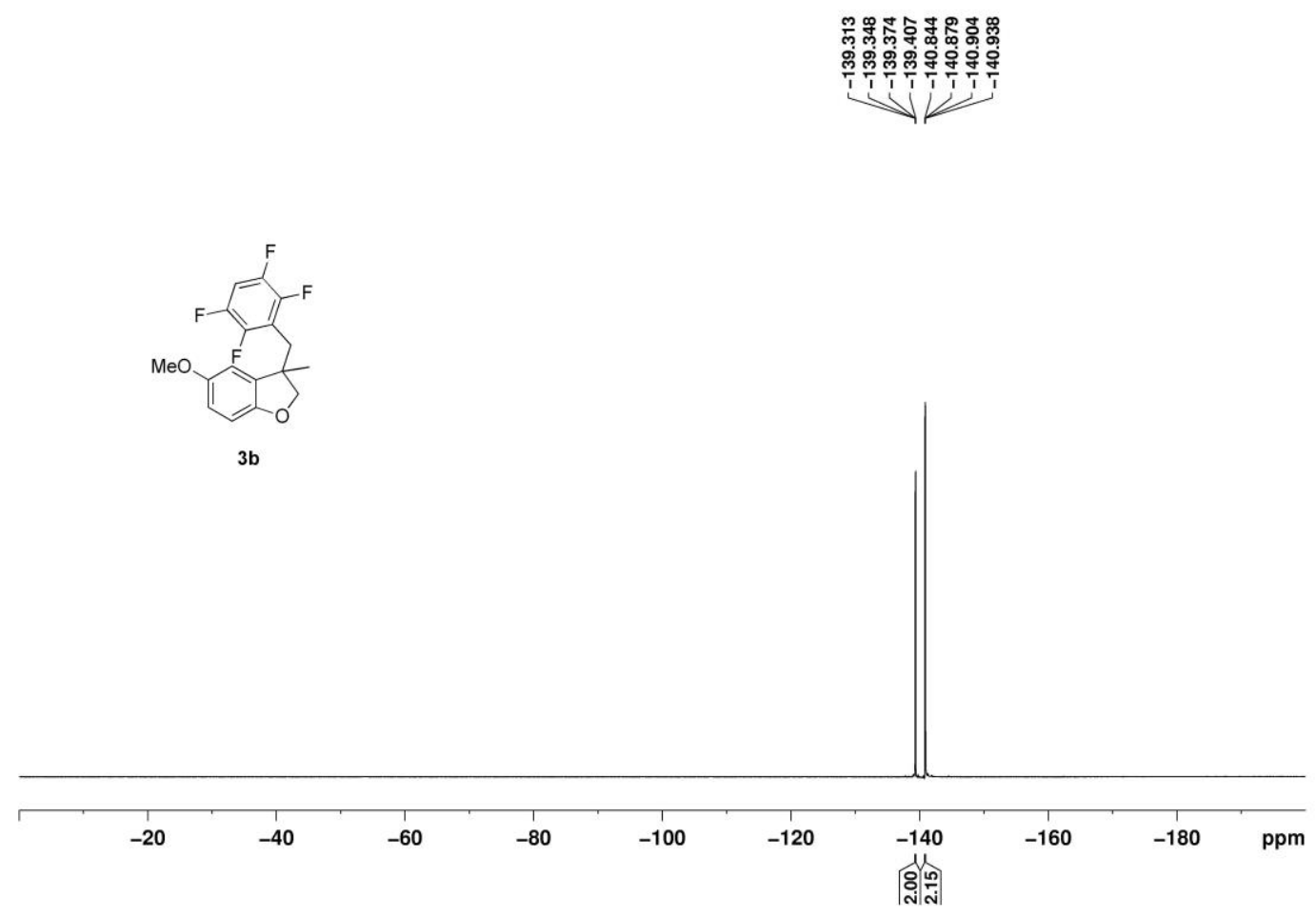




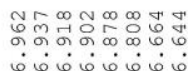

$\dot{0} \dot{0} \dot{0} \dot{0} \dot{0}$

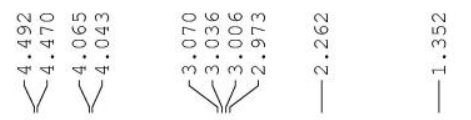

$\circ$
$\vdots$
$i$

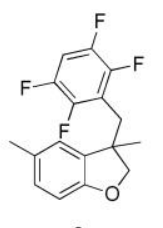

$3 c$

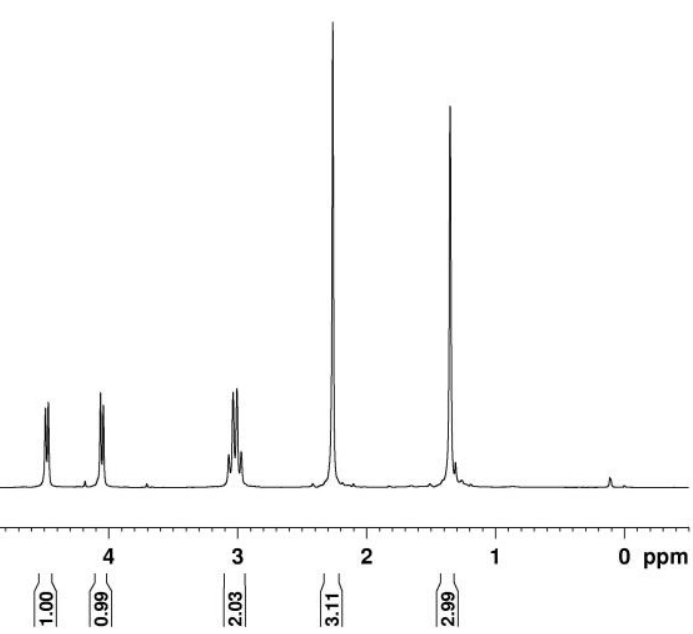

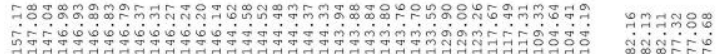

VฟV

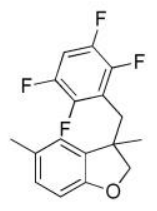

$3 c$

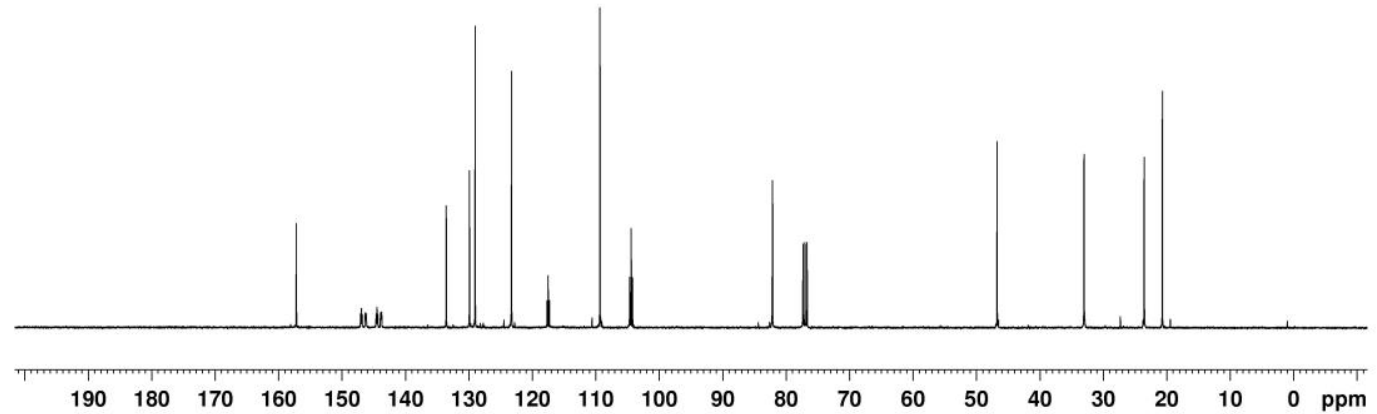




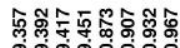

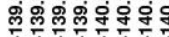

N

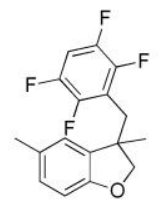

3c

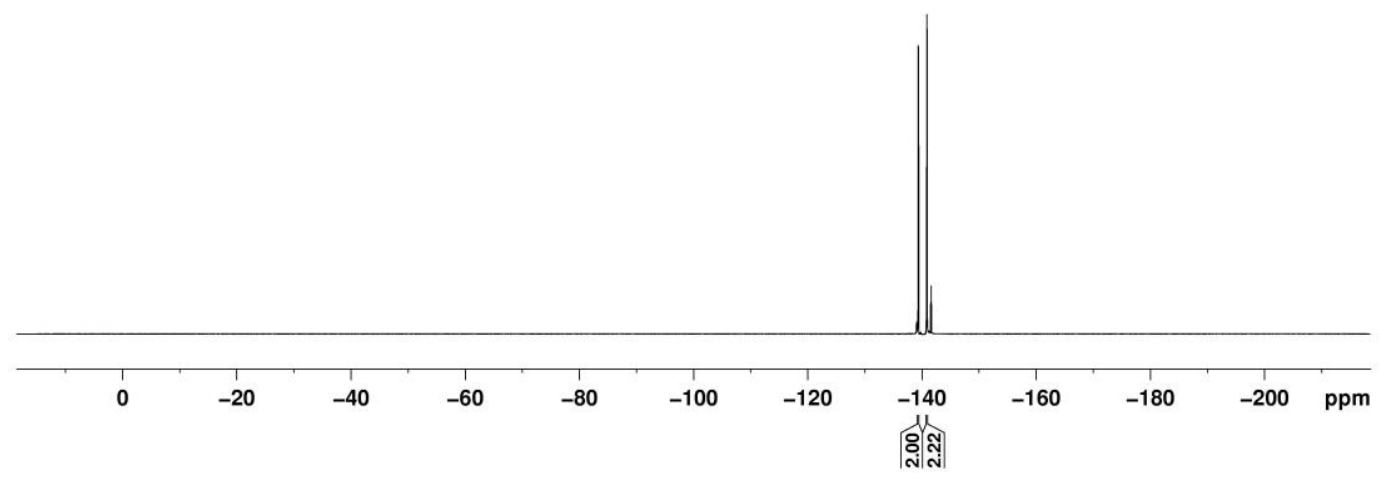

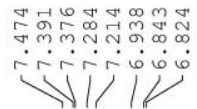

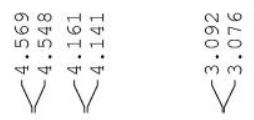
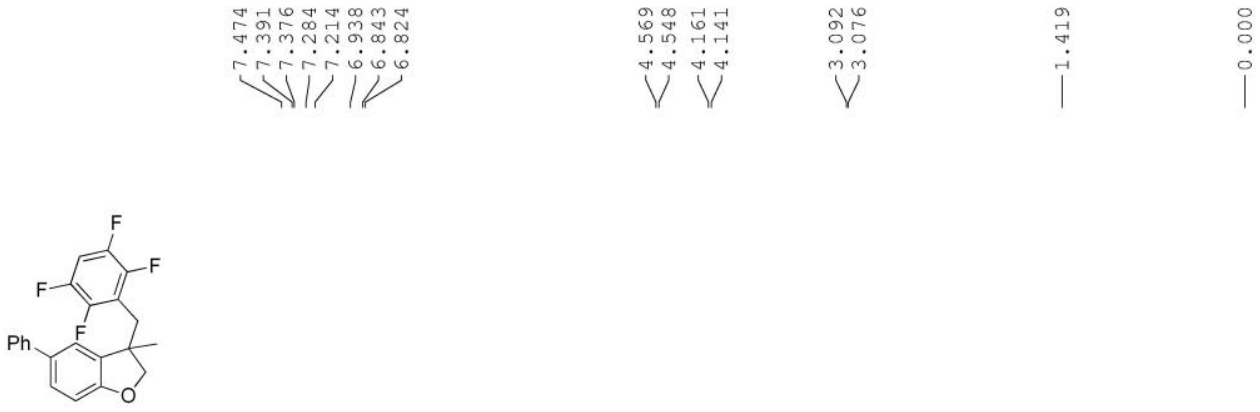

3d

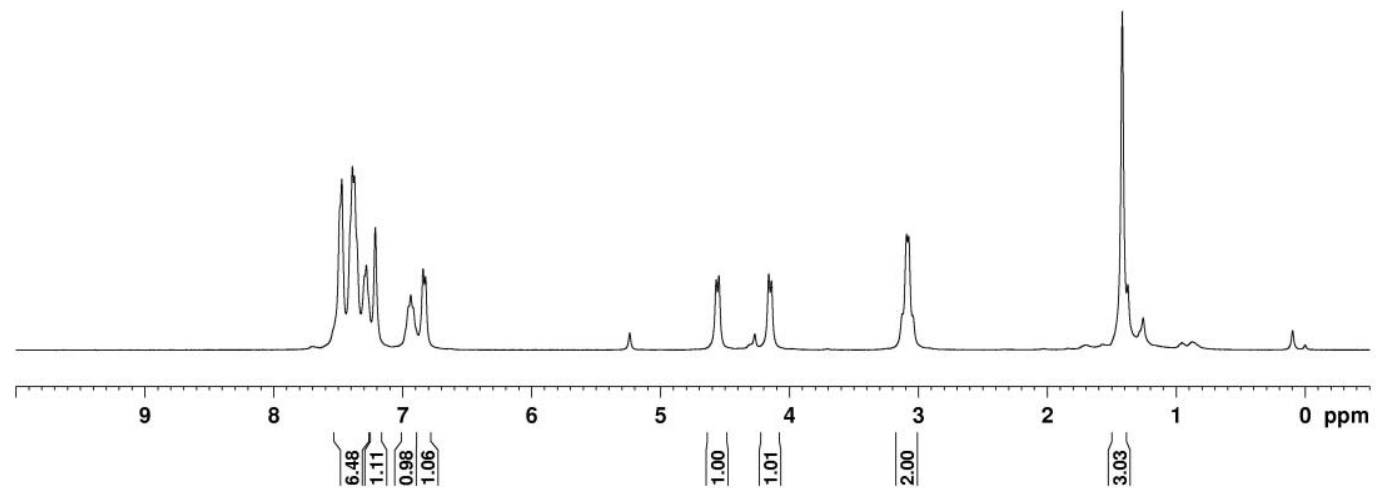




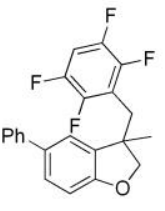

3d

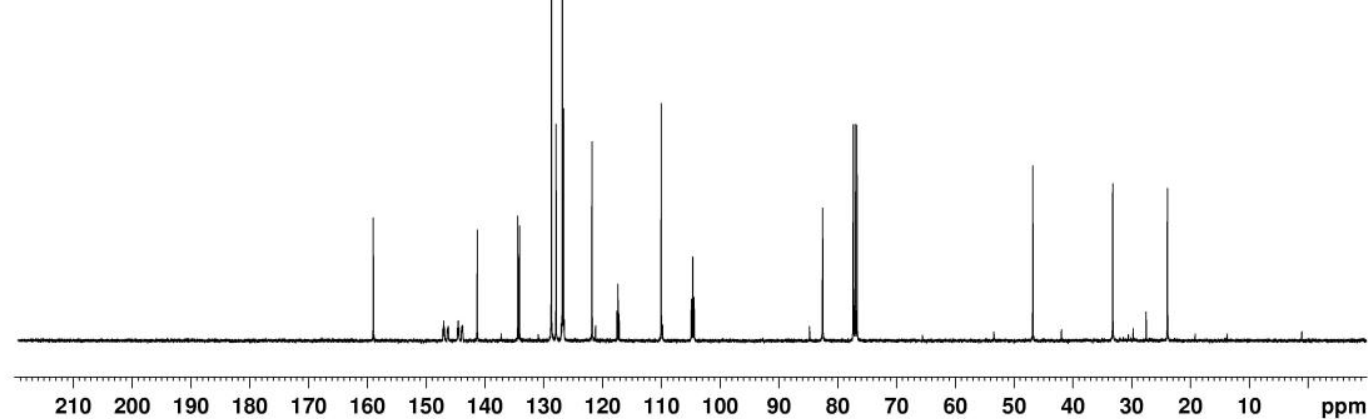

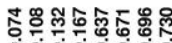

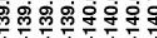

Nin'

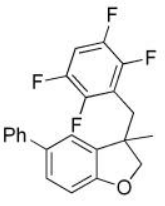

3d

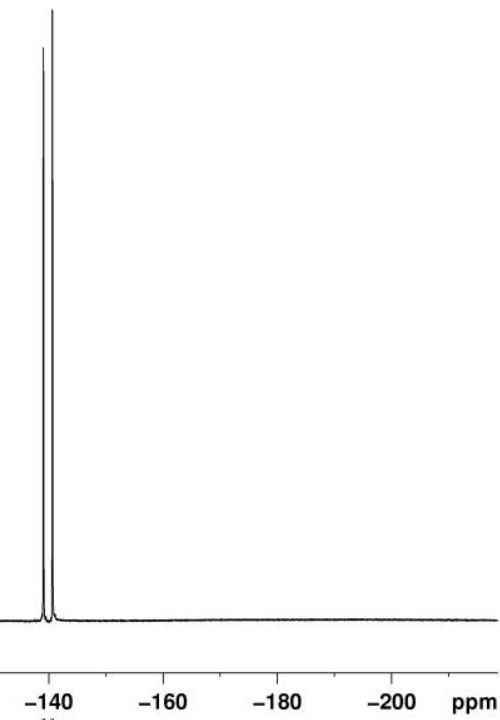

悀 

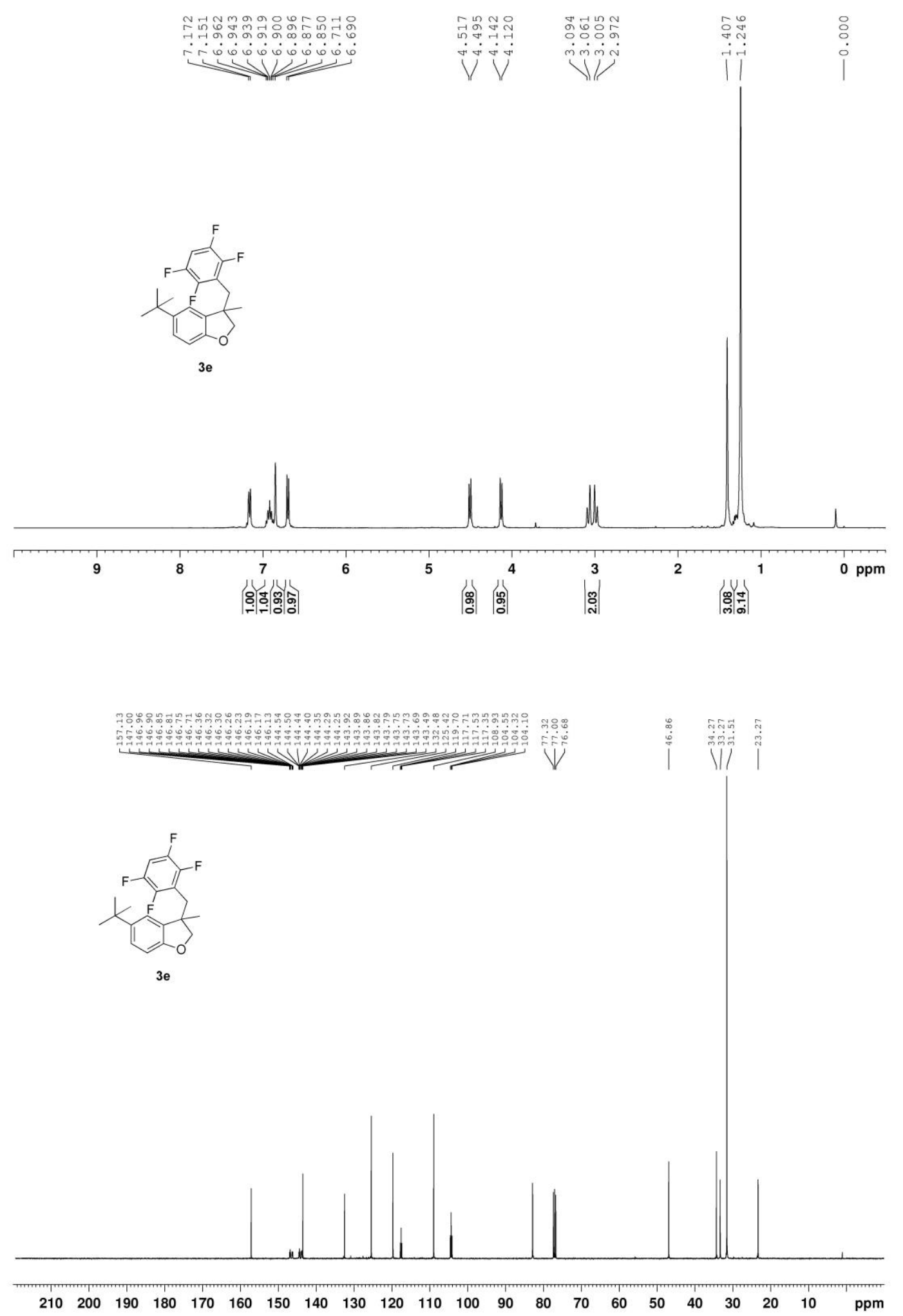


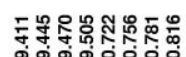

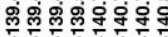

议

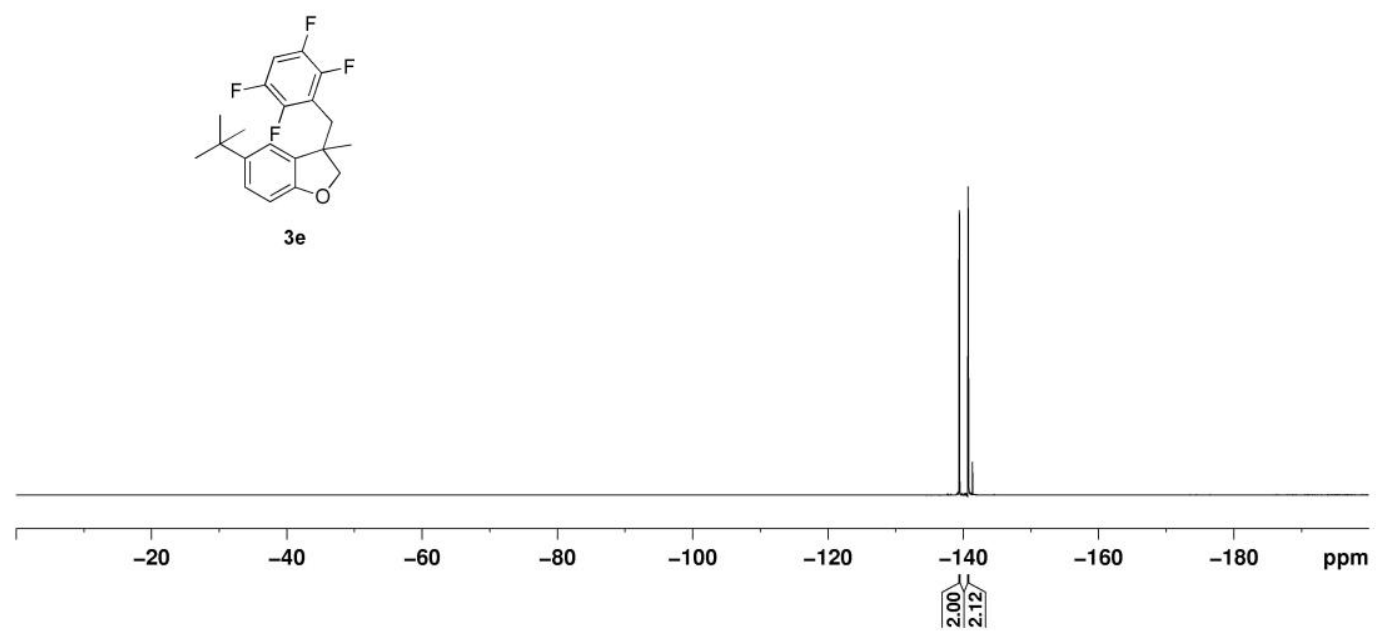

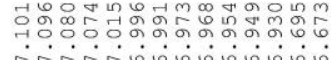

rratrogo
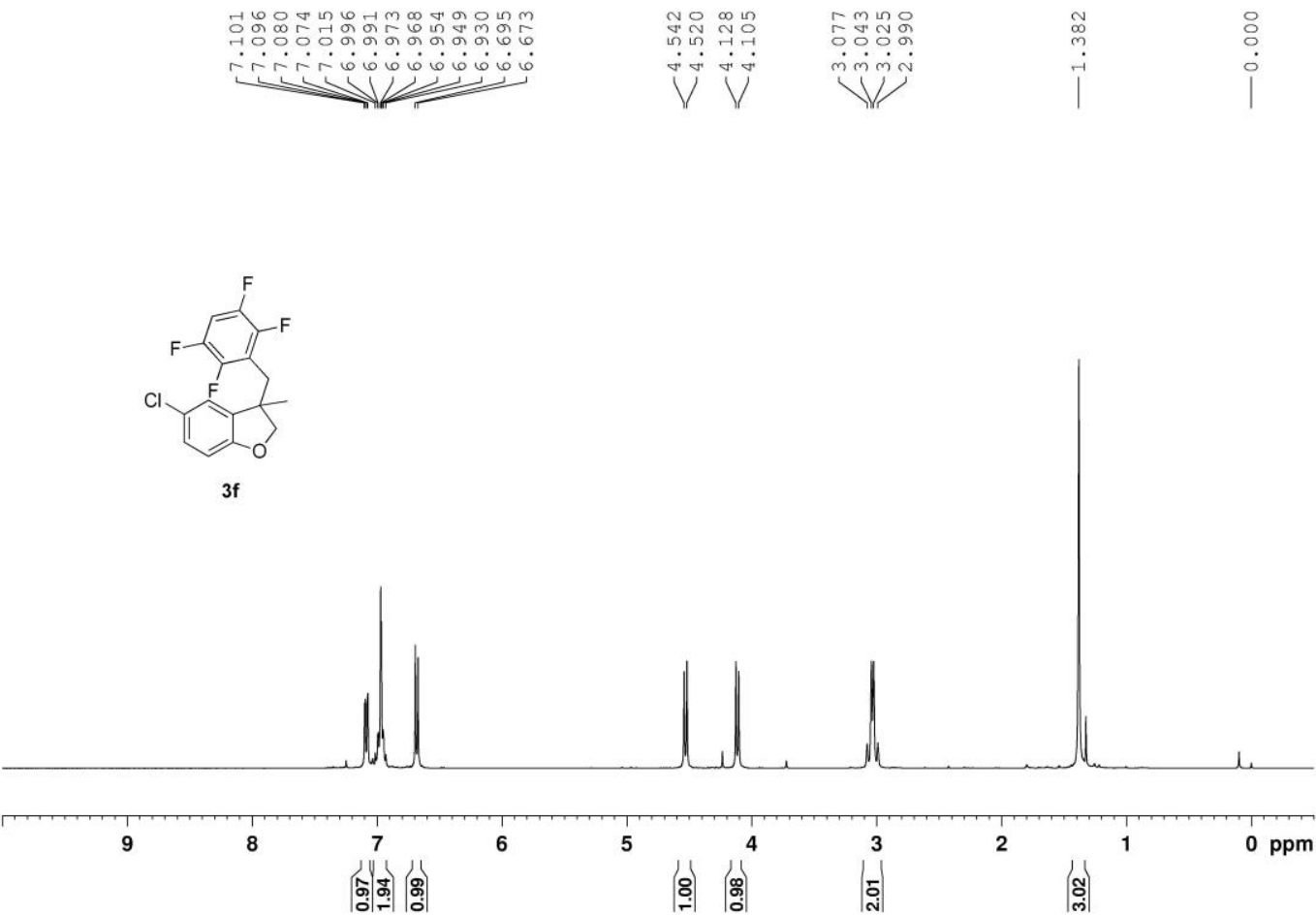


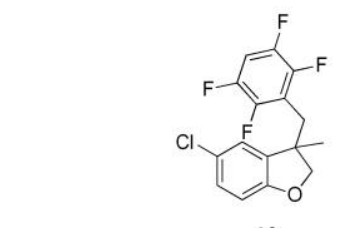

$3 \mathbf{f}$

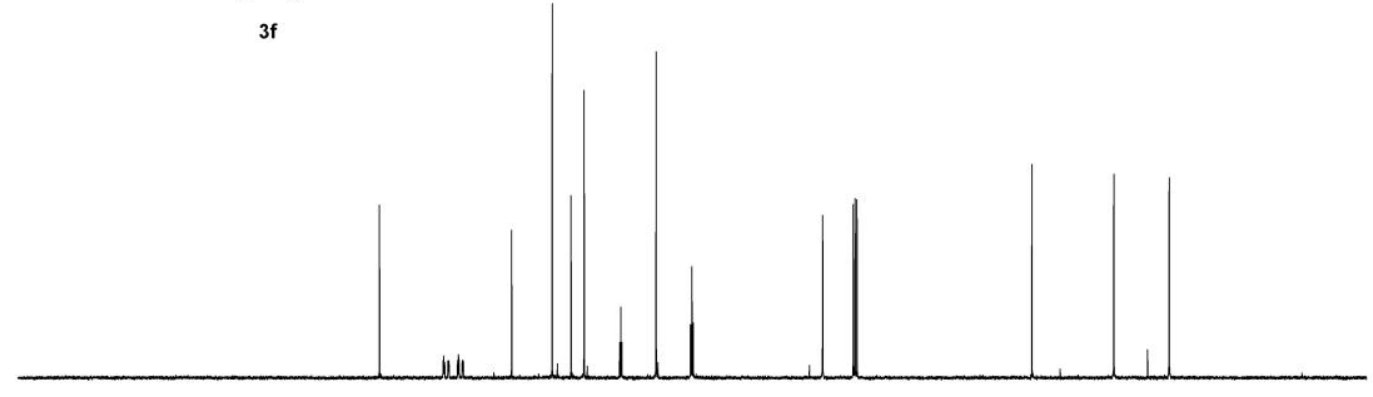

$\begin{array}{llllllllllllllllllllll}210 & 200 & 190 & 180 & 170 & 160 & 150 & 140 & 130 & 120 & 110 & 100 & 90 & 80 & 70 & 60 & 50 & 40 & 30 & 20 & 10 & \mathrm{ppm}\end{array}$

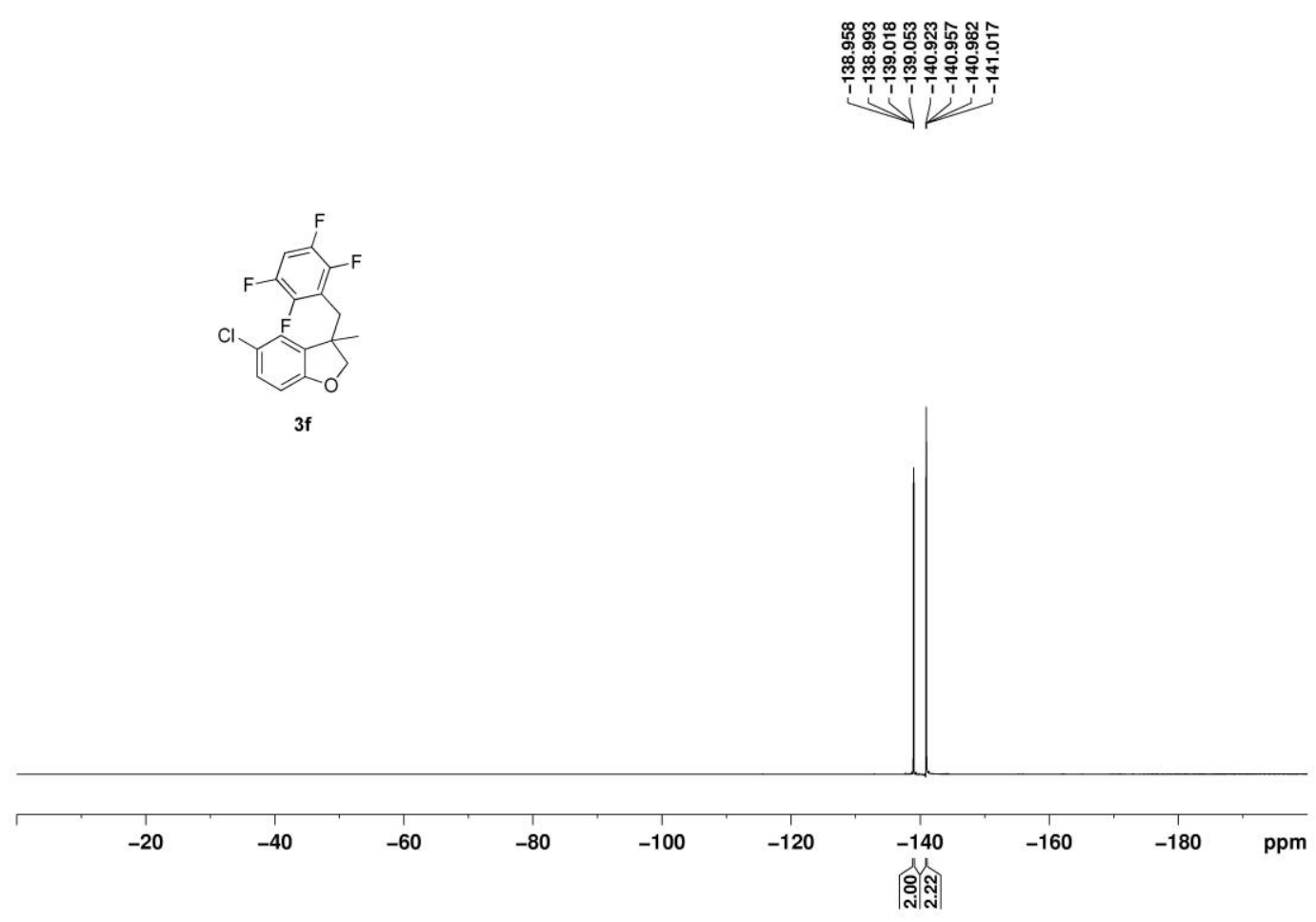




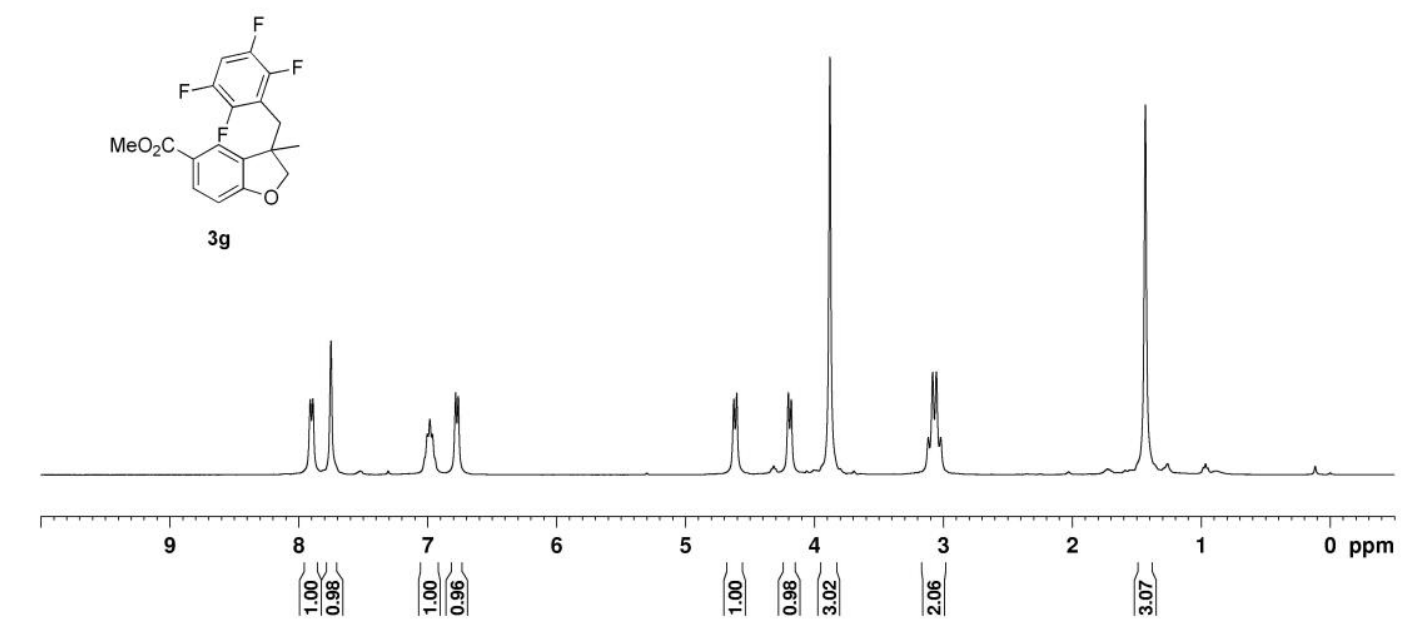

$3 \mathrm{~g}$

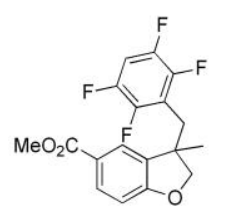

$3 \mathrm{~g}$

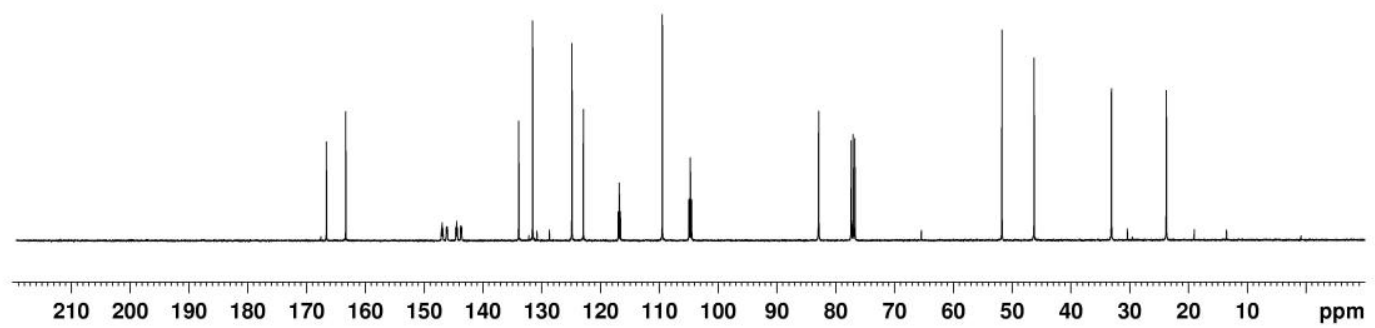




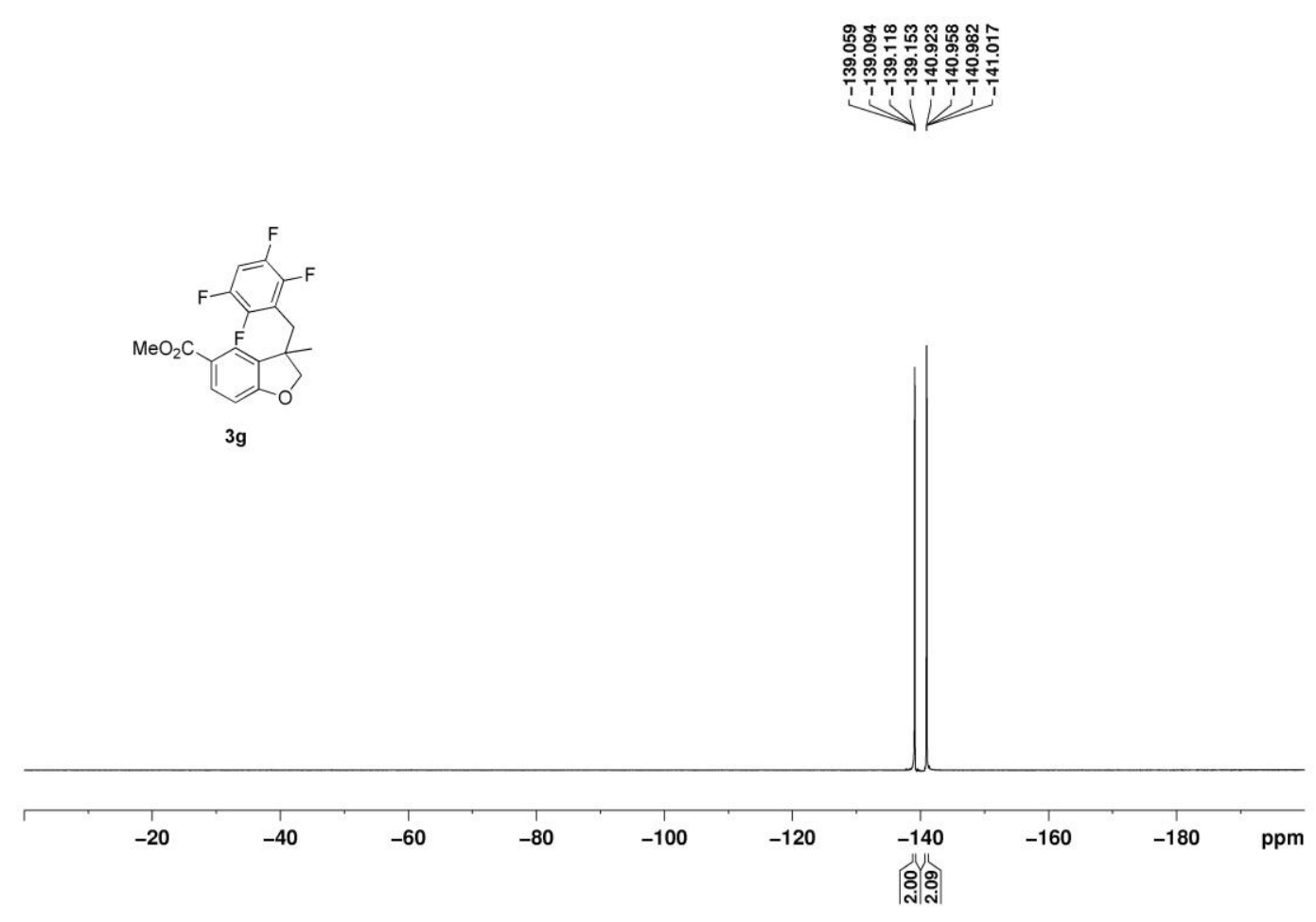

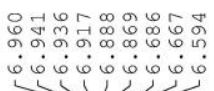

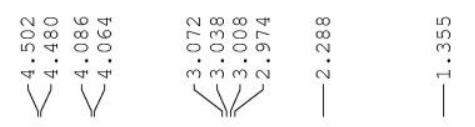

$\circ$
$\vdots$
$\vdots$
$i$

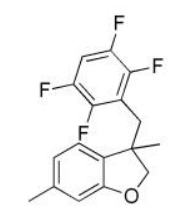

$3 \mathrm{~h}$

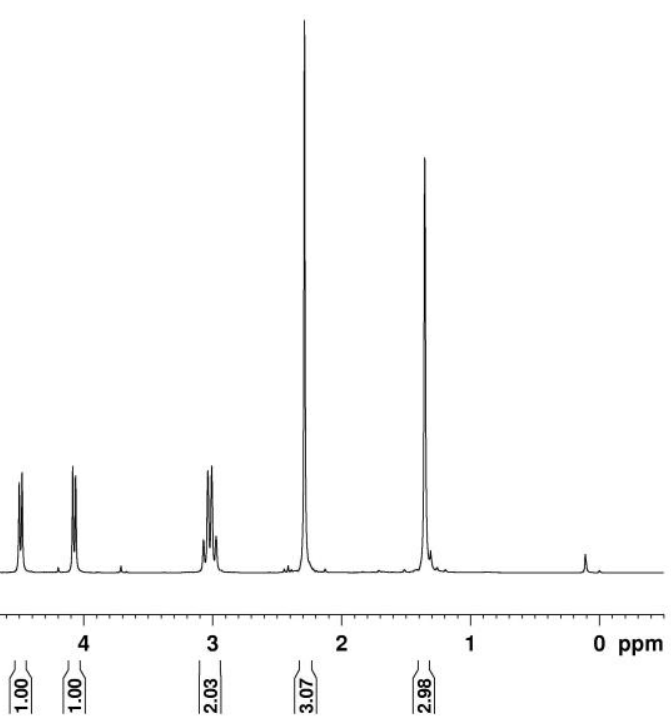




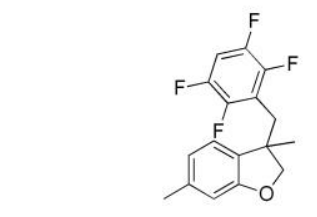

$3 \mathrm{~h}$

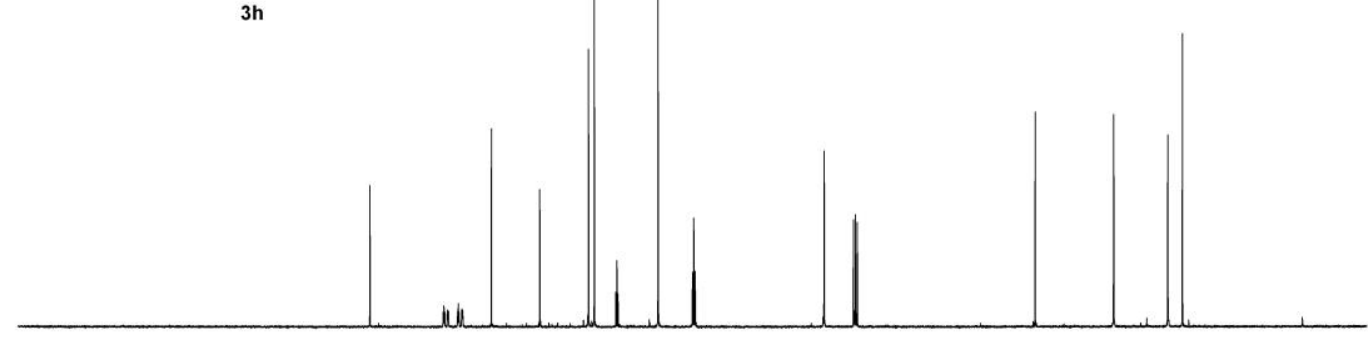

$\begin{array}{llllllllllllllllllllll}210 & 200 & 190 & 180 & 170 & 160 & 150 & 140 & 130 & 120 & 110 & 100 & 90 & 80 & 70 & 60 & 50 & 40 & 30 & 20 & 10 & \mathrm{ppm}\end{array}$
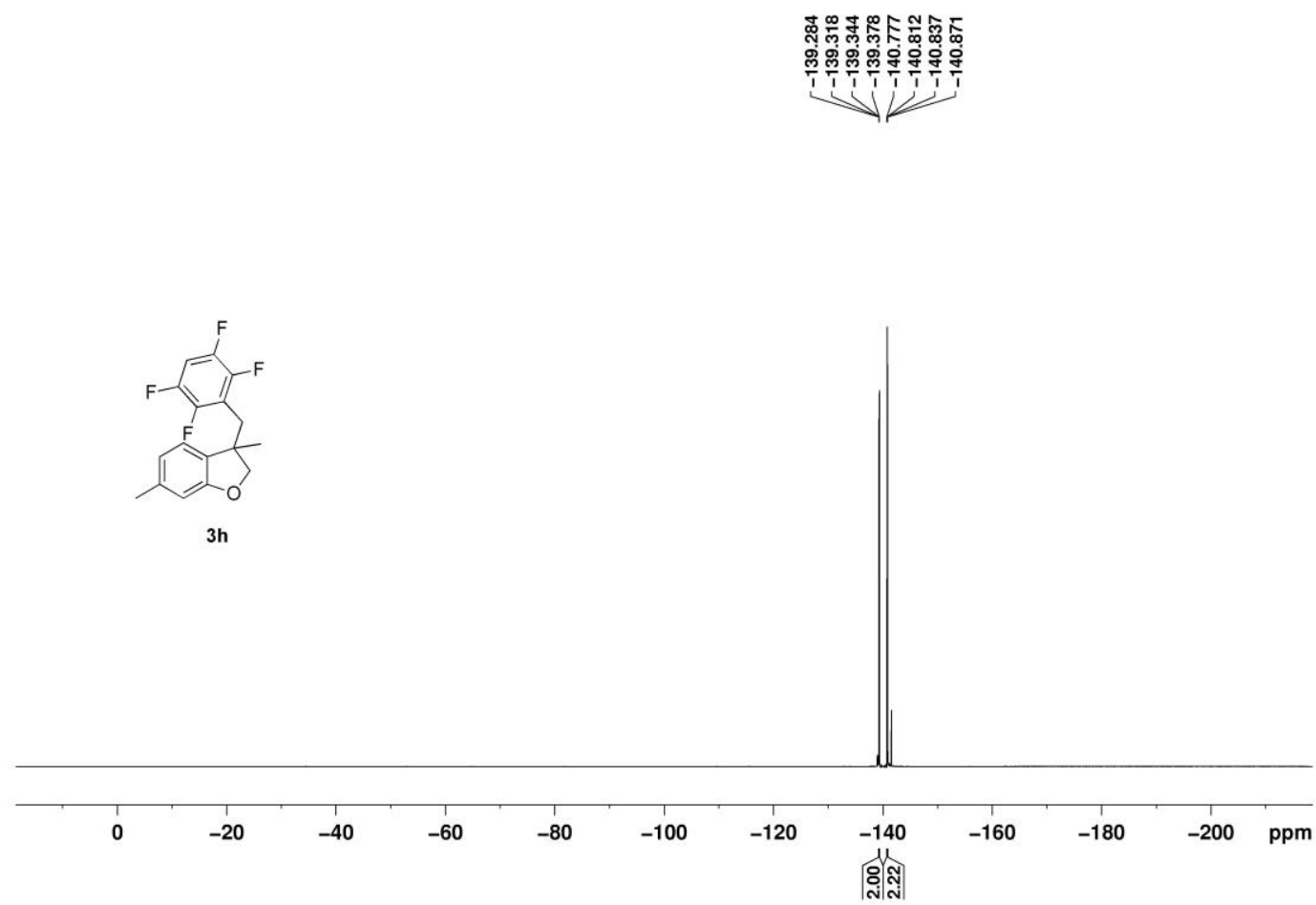


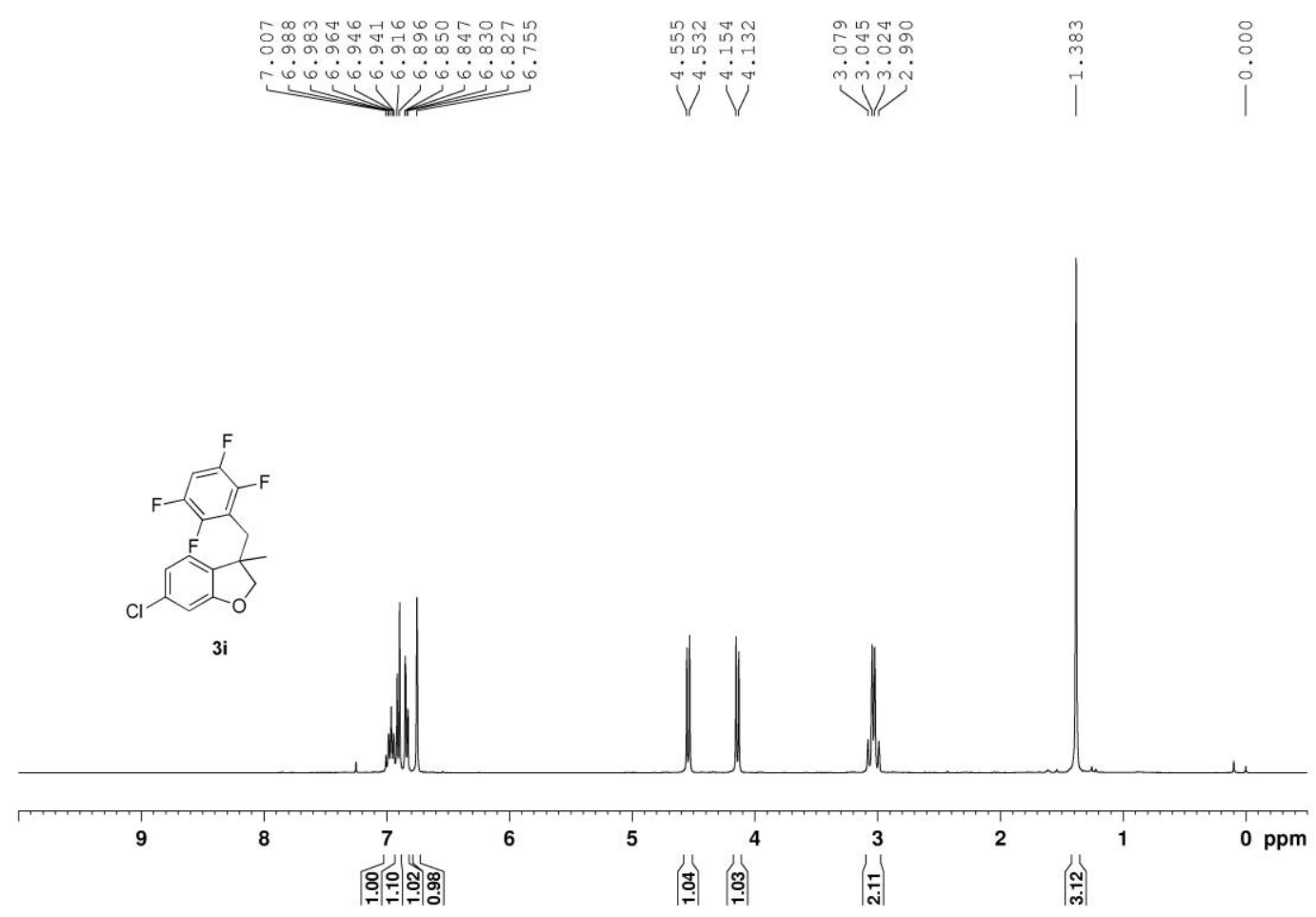

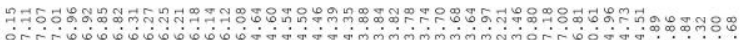
ofrod
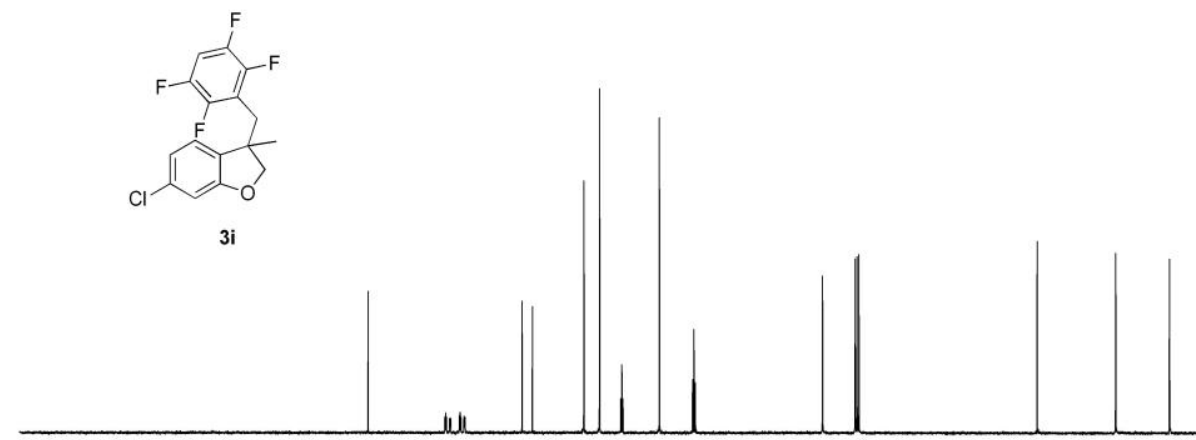

$\begin{array}{llllllllllllllllllllll}210 & 200 & 190 & 180 & 170 & 160 & 150 & 140 & 130 & 120 & 110 & 100 & 90 & 80 & 70 & 60 & 50 & 40 & 30 & 20 & 10 & \mathrm{ppm}\end{array}$ 


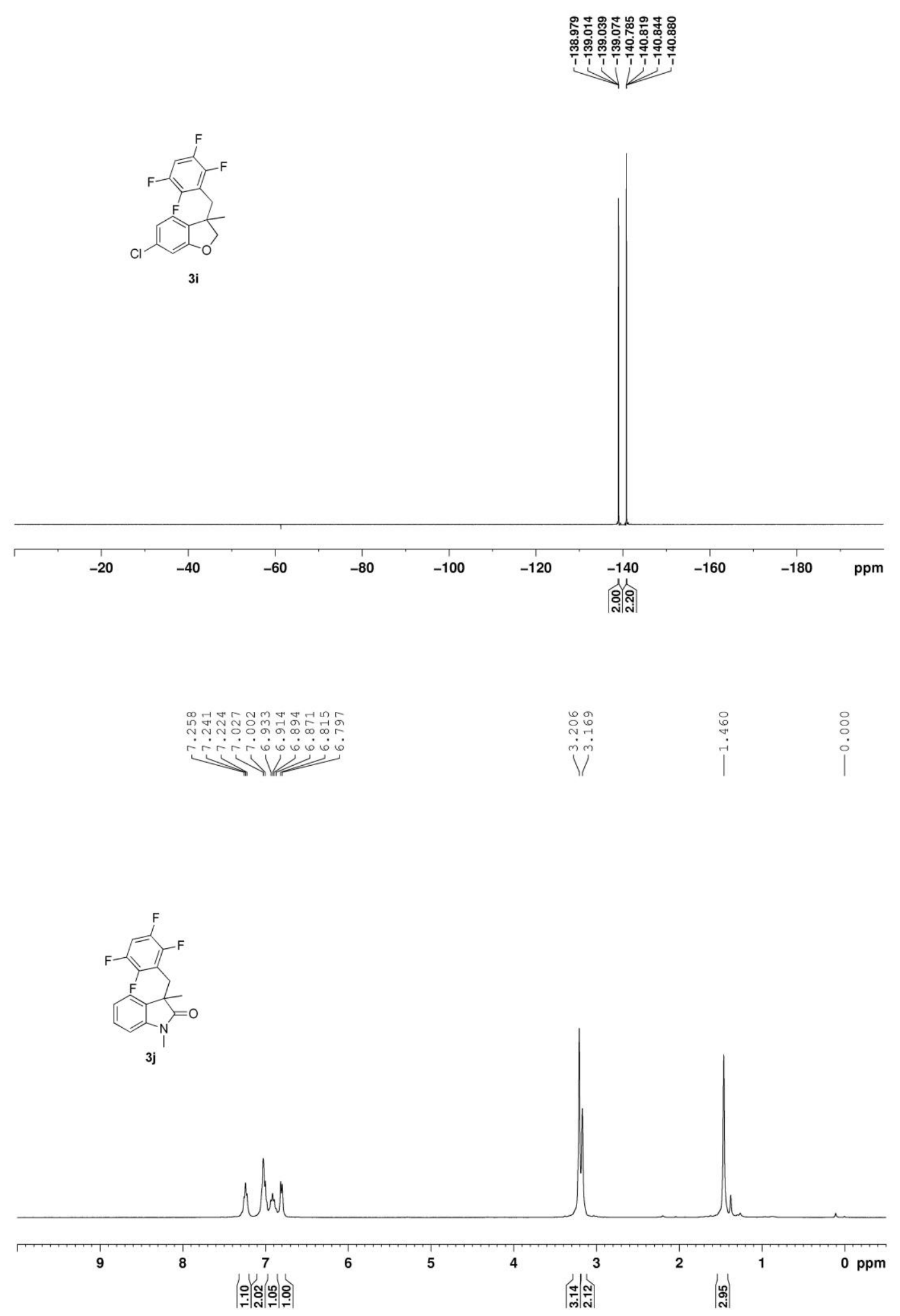



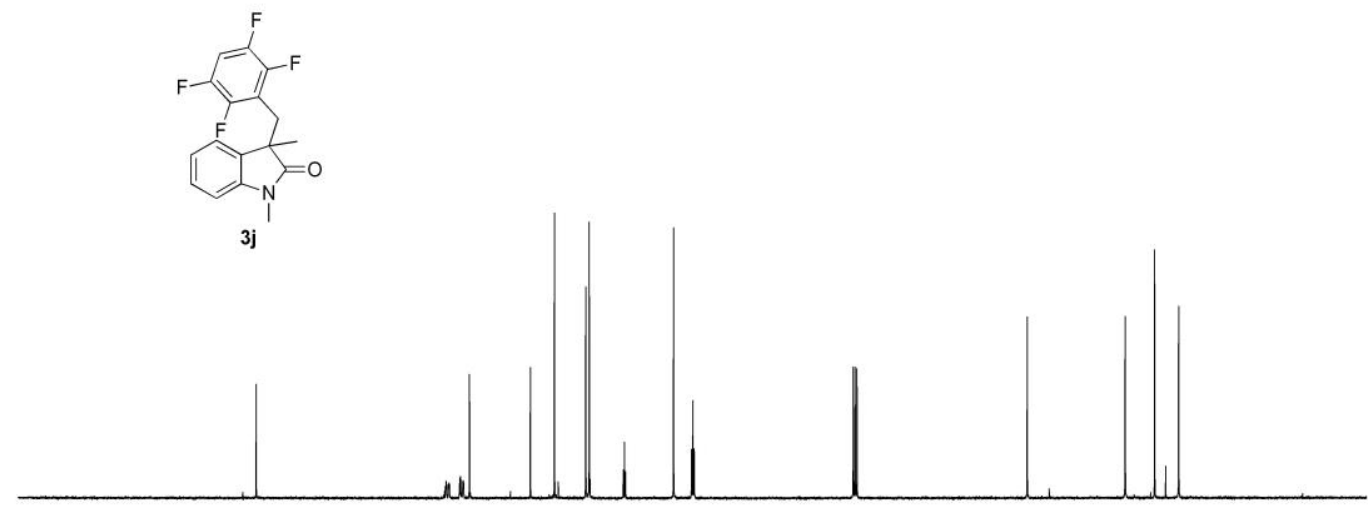

$\begin{array}{lllllllllllllllllllll}210 & 200 & 190 & 180 & 170 & 160 & 150 & 140 & 130 & 120 & 110 & 100 & 90 & 80 & 70 & 60 & 50 & 40 & 30 & 20 & 10\end{array}$

\section{兽}

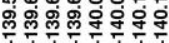

inisi

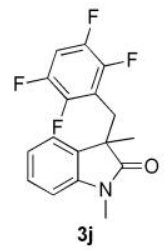

3j

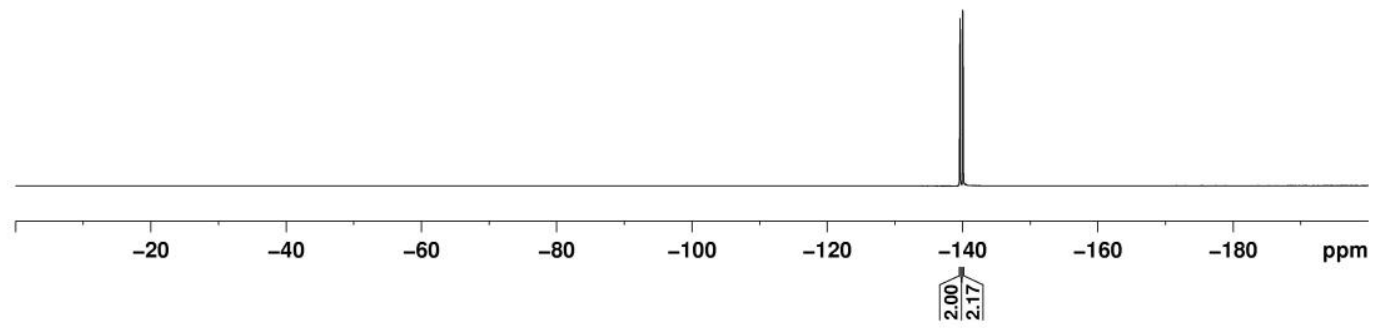




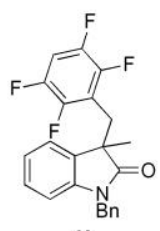

$3 \mathbf{k}$

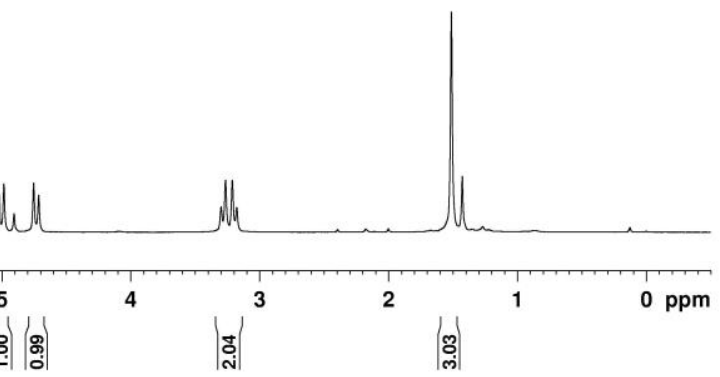

$\underbrace{\text { 9. }}$

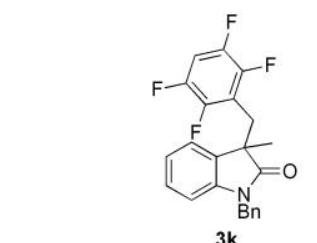

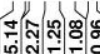

훙

$\mid$

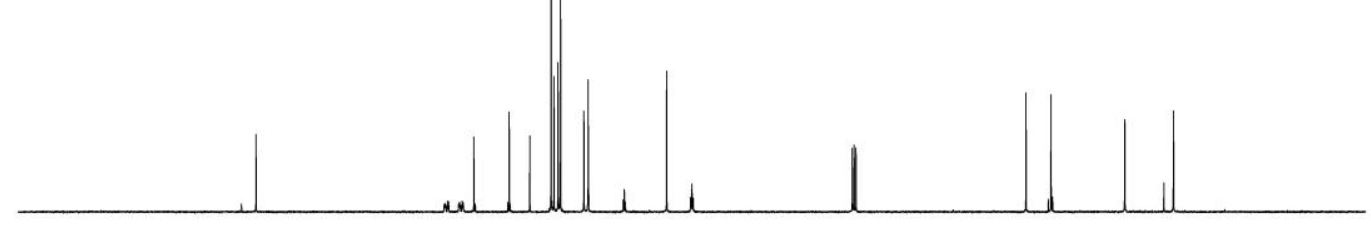

$\begin{array}{llllllllllllllllllllll}210 & 200 & 190 & 180 & 170 & 160 & 150 & 140 & 130 & 120 & 110 & 100 & 90 & 80 & 70 & 60 & 50 & 40 & 30 & 20 & 10 & \mathrm{ppm}\end{array}$ 


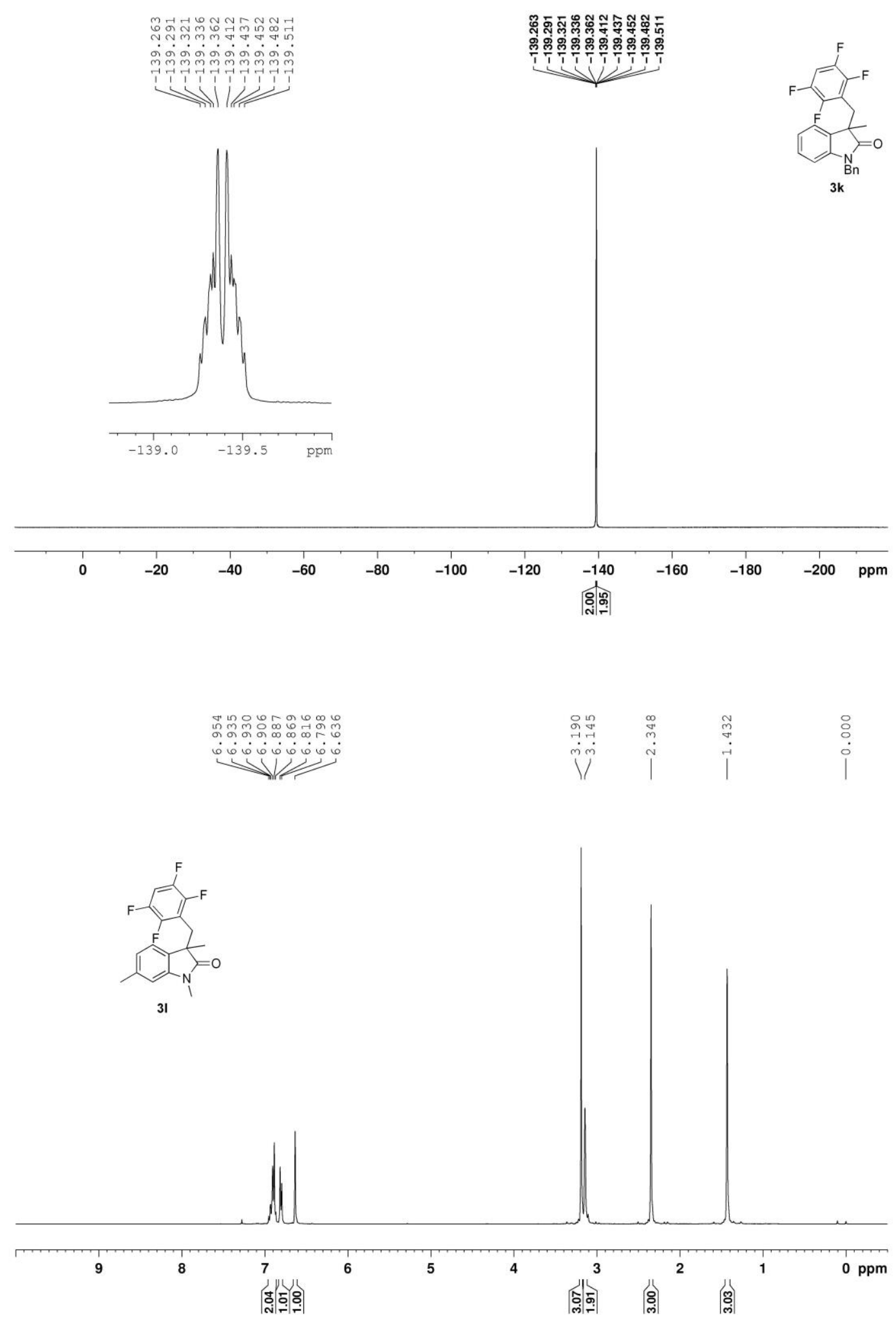




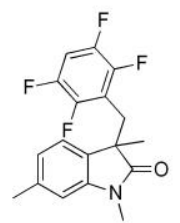

31

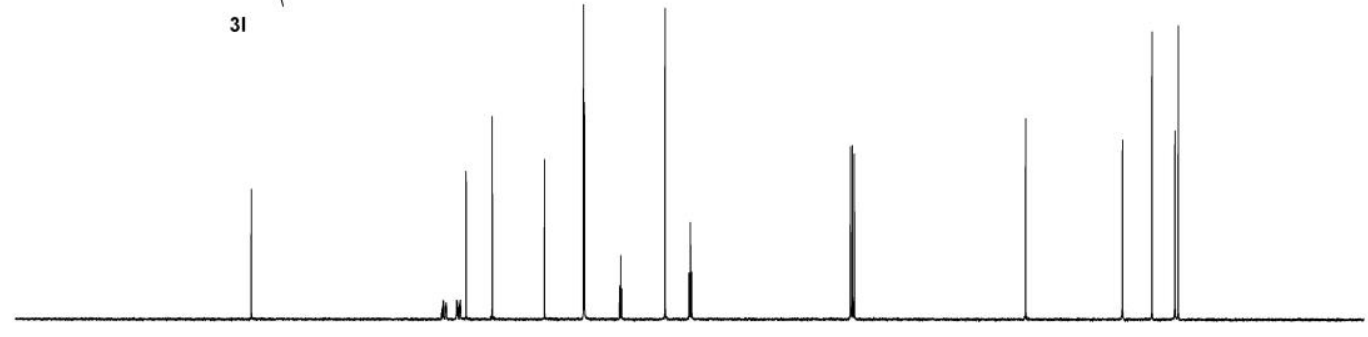

$\begin{array}{llllllllllllllllllllll}210 & 200 & 190 & 180 & 170 & 160 & 150 & 140 & 130 & 120 & 110 & 100 & 90 & 80 & 70 & 60 & 50 & 40 & 30 & 20 & 10 & \mathrm{ppm}\end{array}$

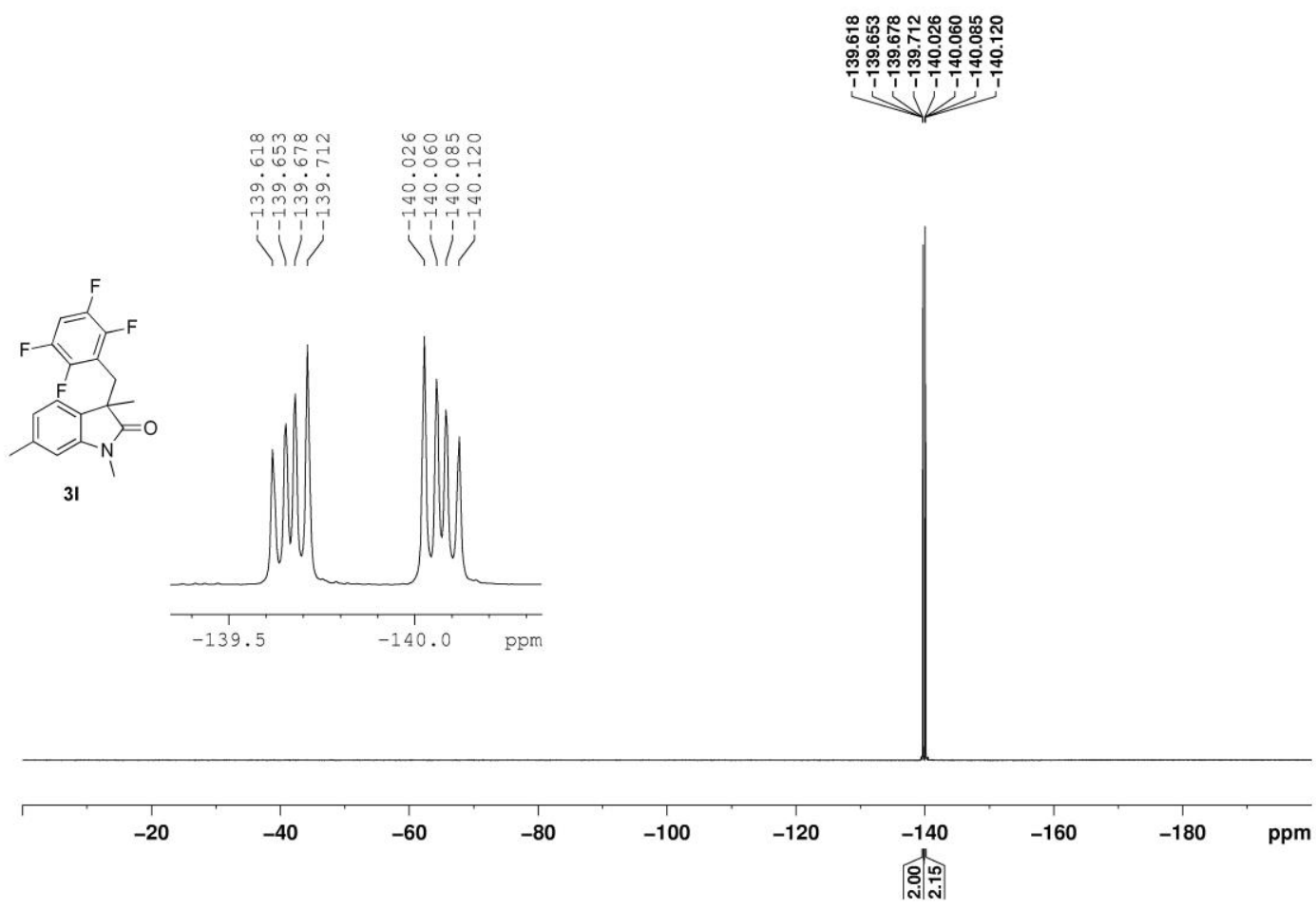




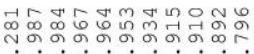

.

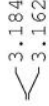

$\stackrel{\circ}{\circ}$

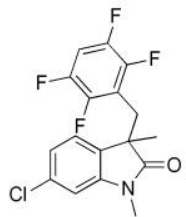

$3 \mathrm{~m}$

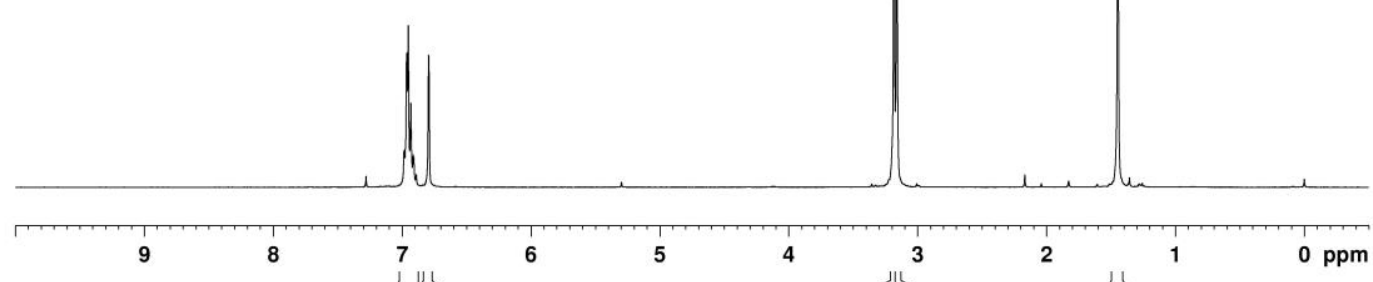

송ำ:

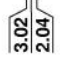

|

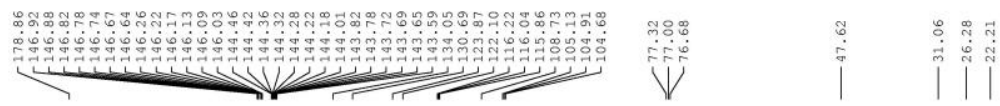

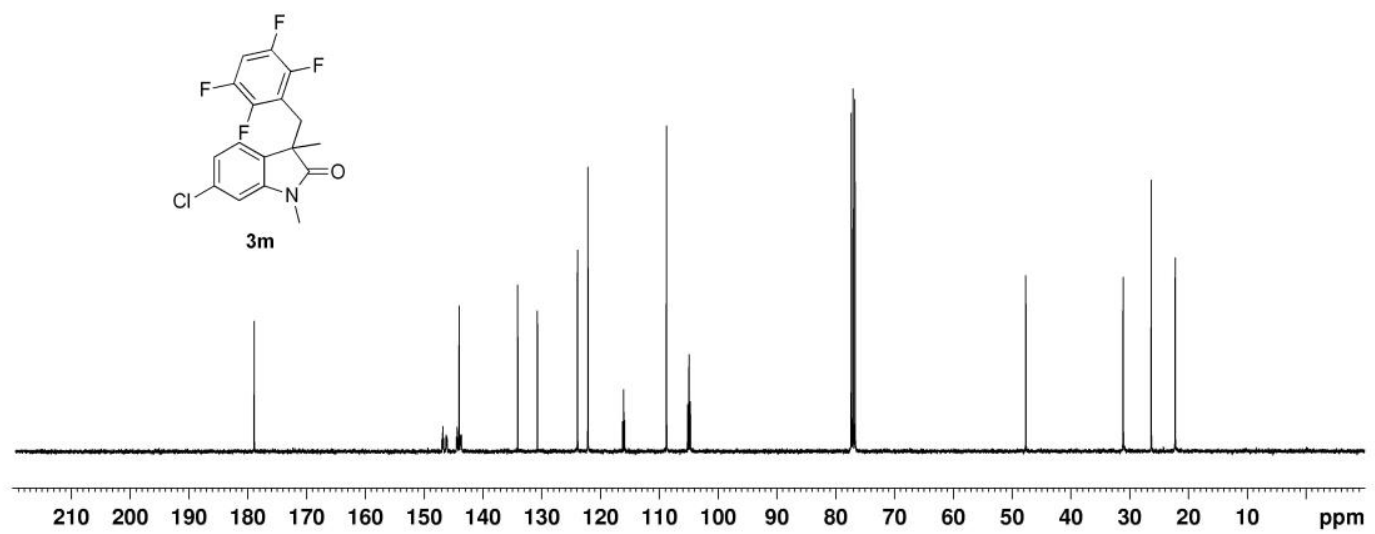




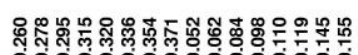

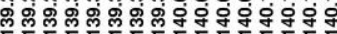

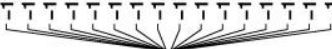
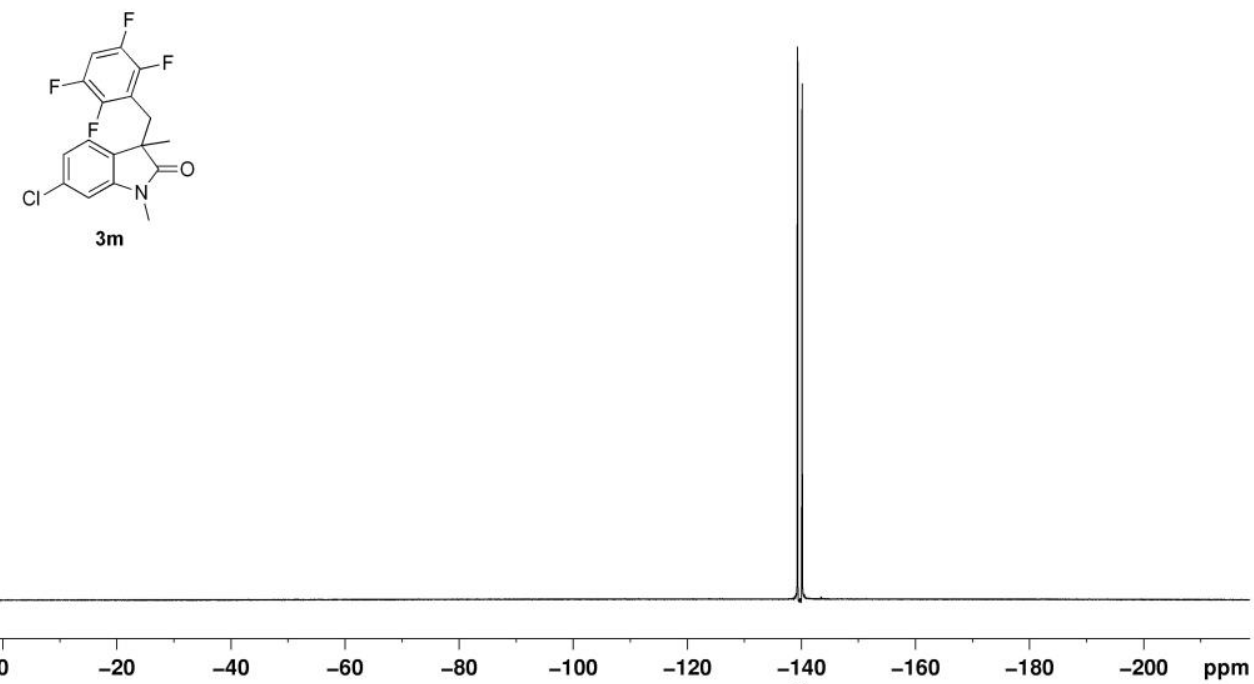

角啇

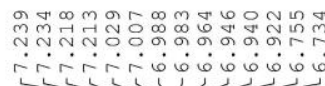

ขึ

|ึ.

ì
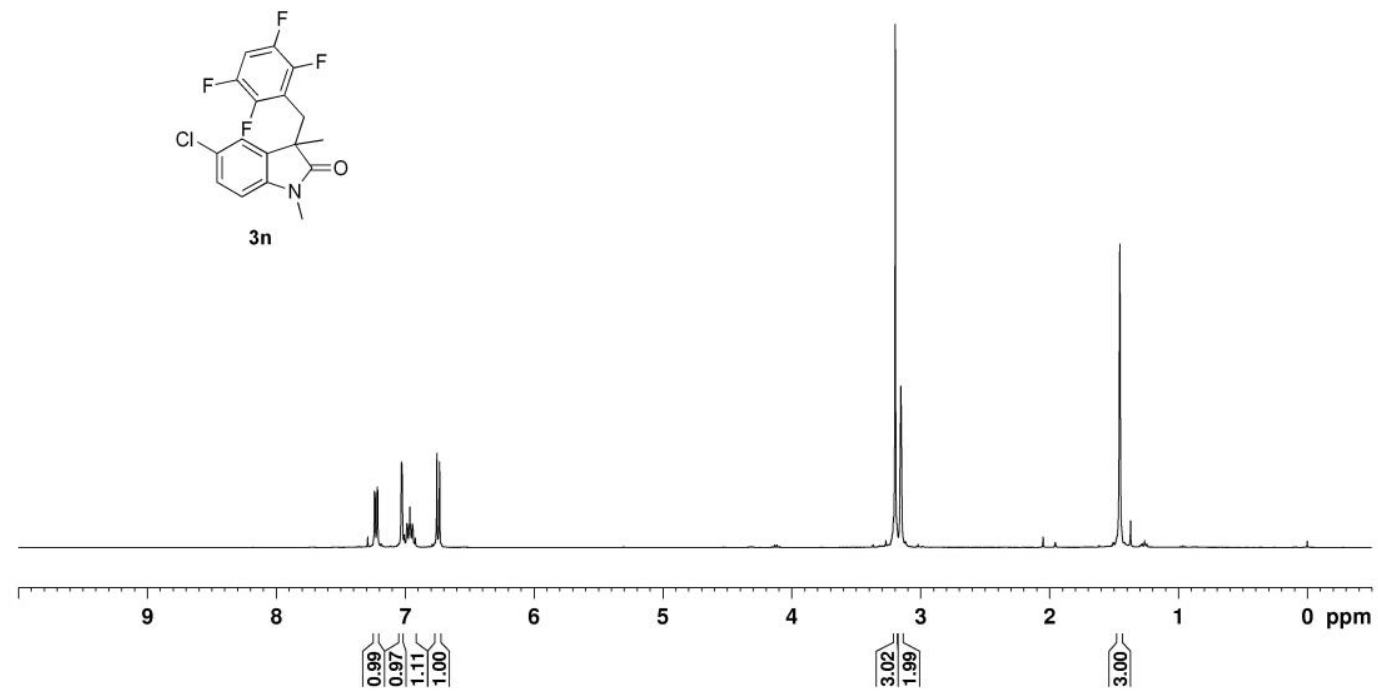


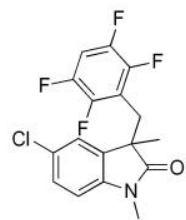

3n

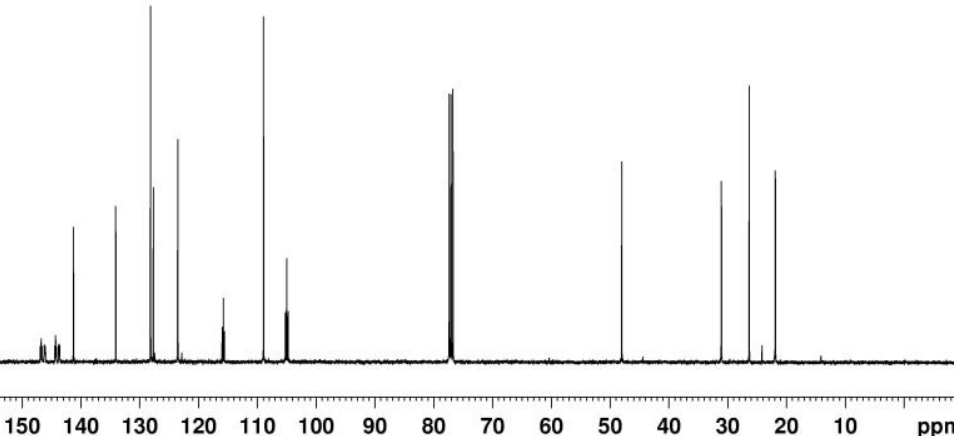

$\begin{array}{llllllllllllllllllllll}210 & 200 & 190 & 180 & 170 & 160 & 150 & 140 & 130 & 120 & 110 & 100 & 90 & 80 & 70 & 60 & 50 & 40 & 30 & 20 & 10 & \mathrm{ppm}\end{array}$

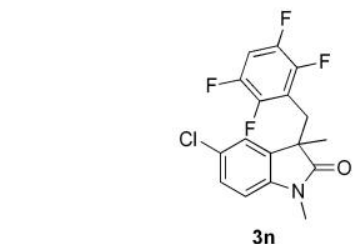

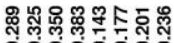

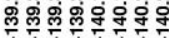

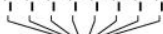

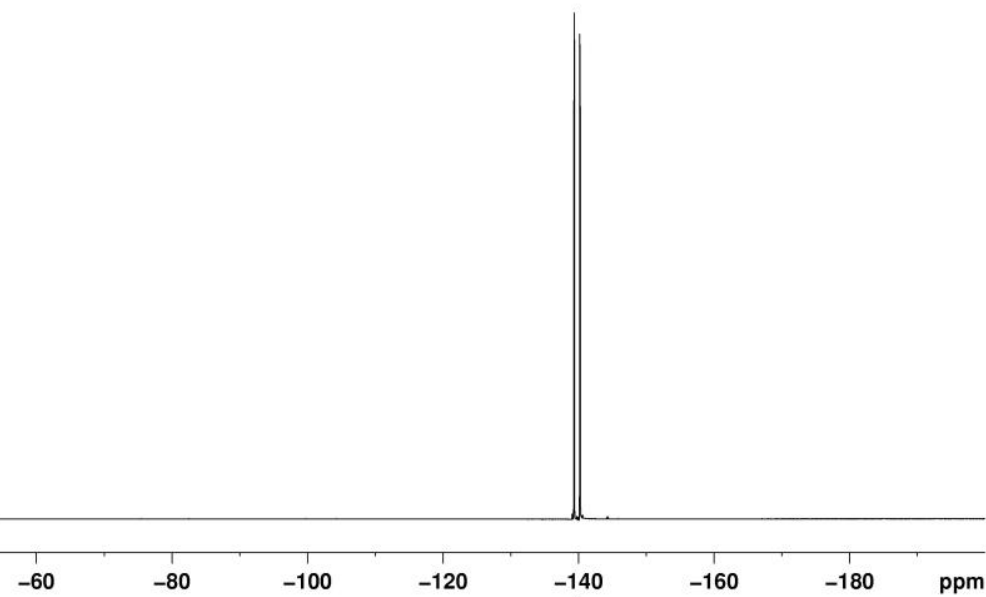

$-20$

$-40$

চึ: 

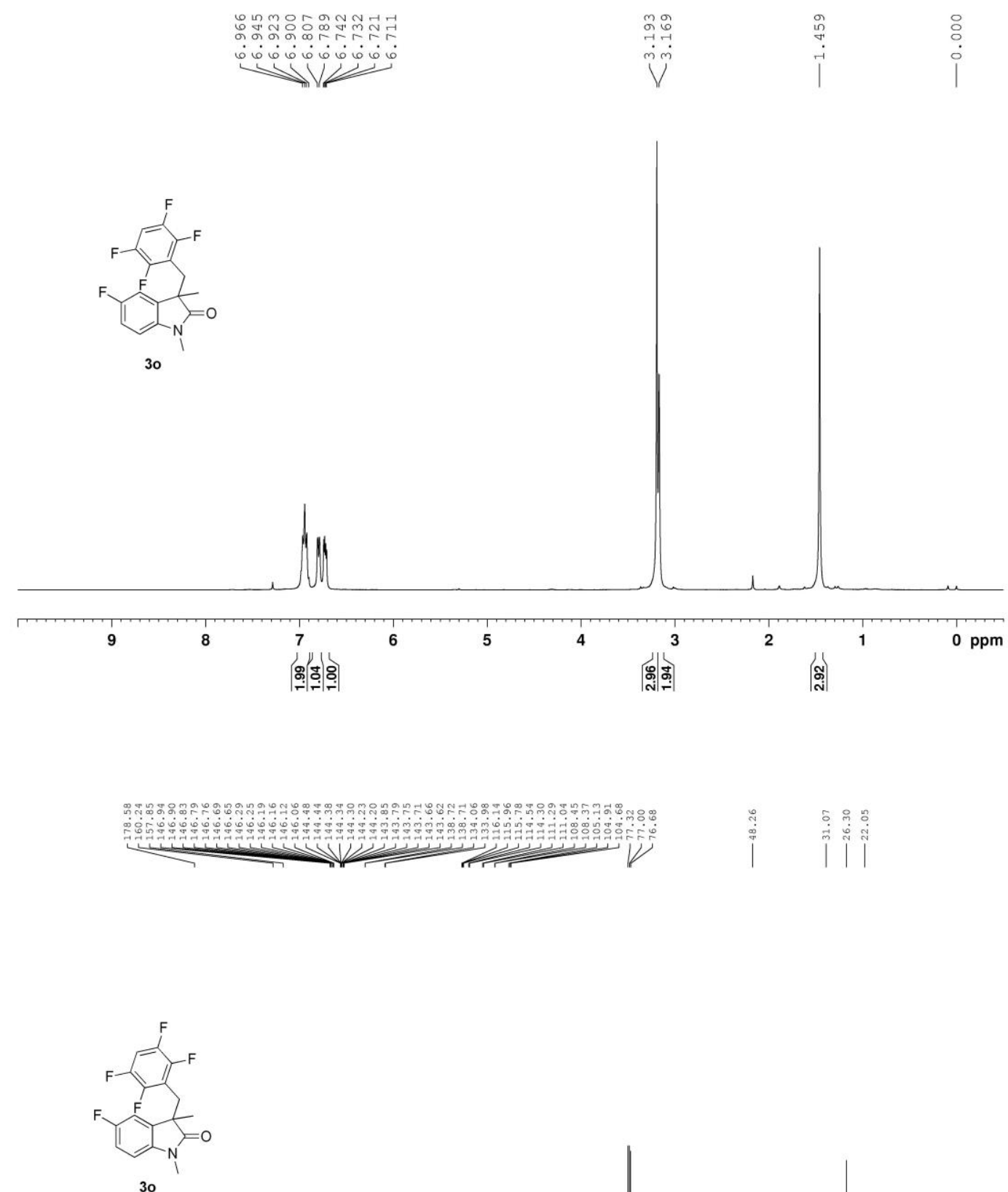

30

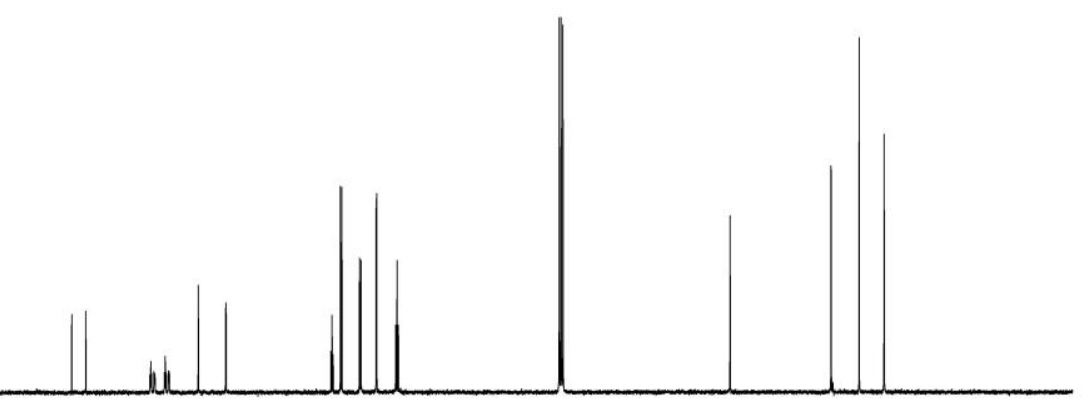

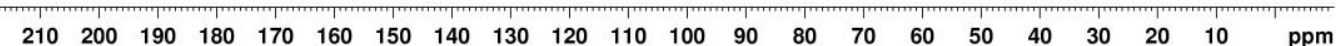




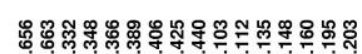

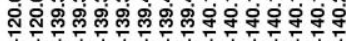

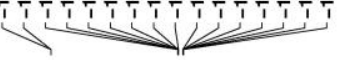

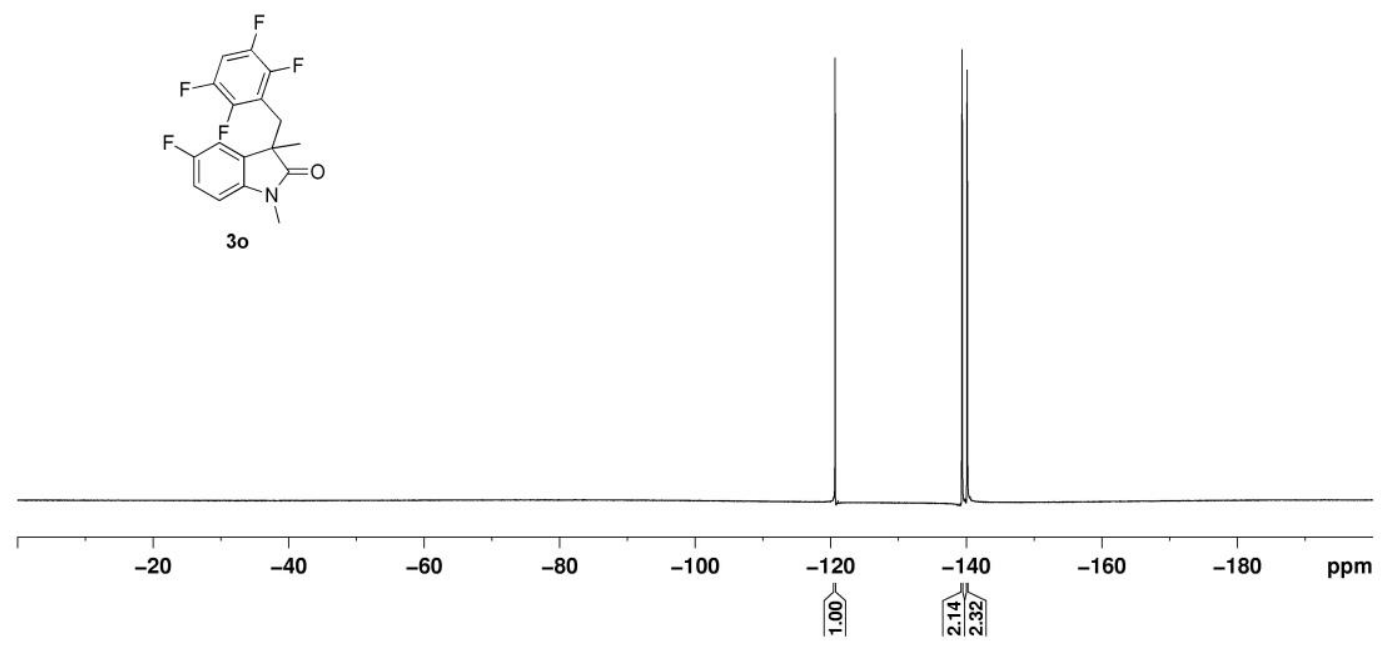

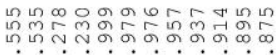

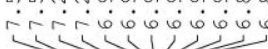
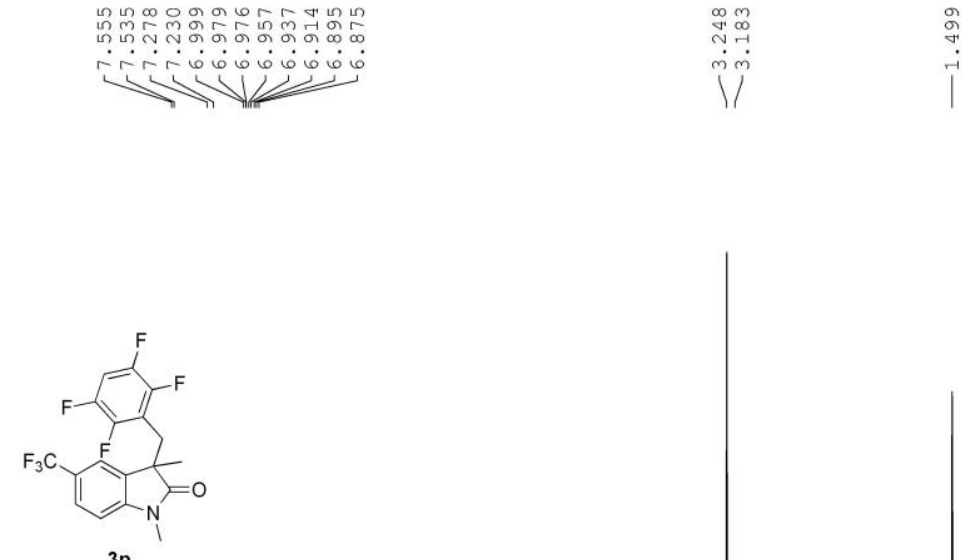

$3 p$

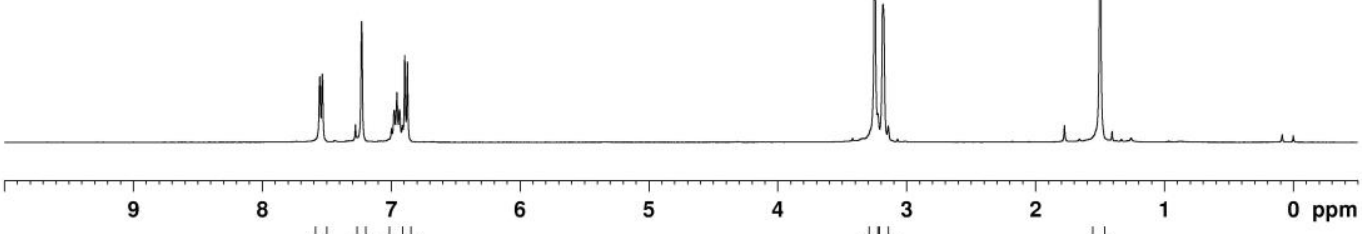

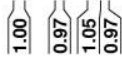

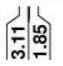

(ֻ) 


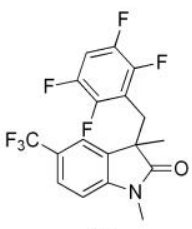

$3 p$

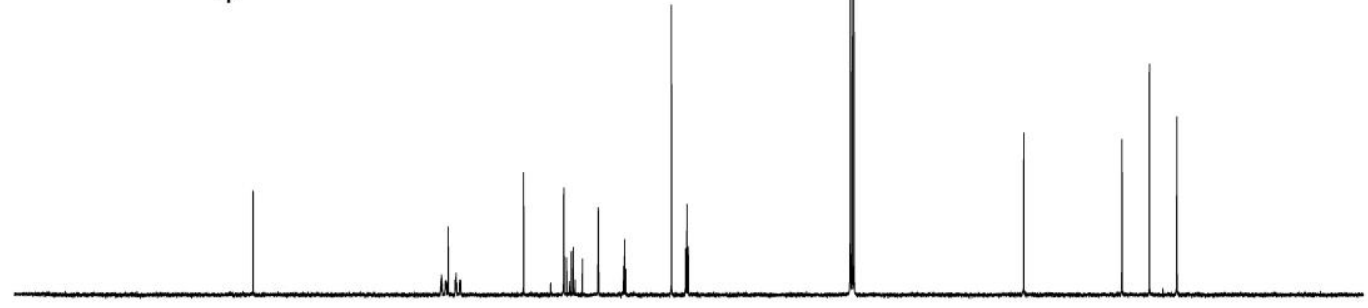

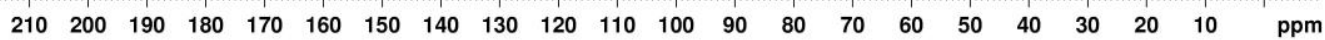

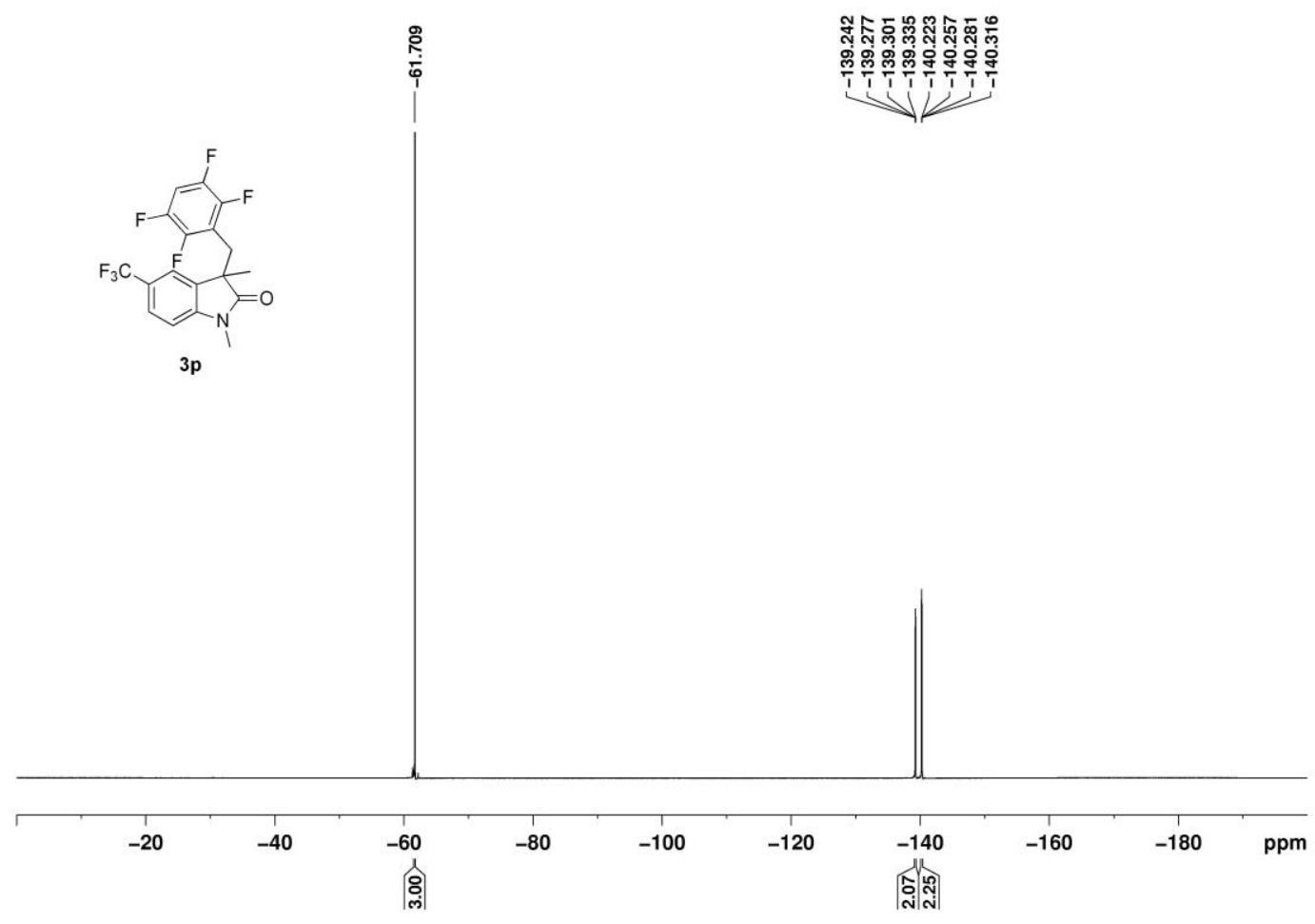




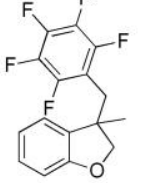

$4 a$
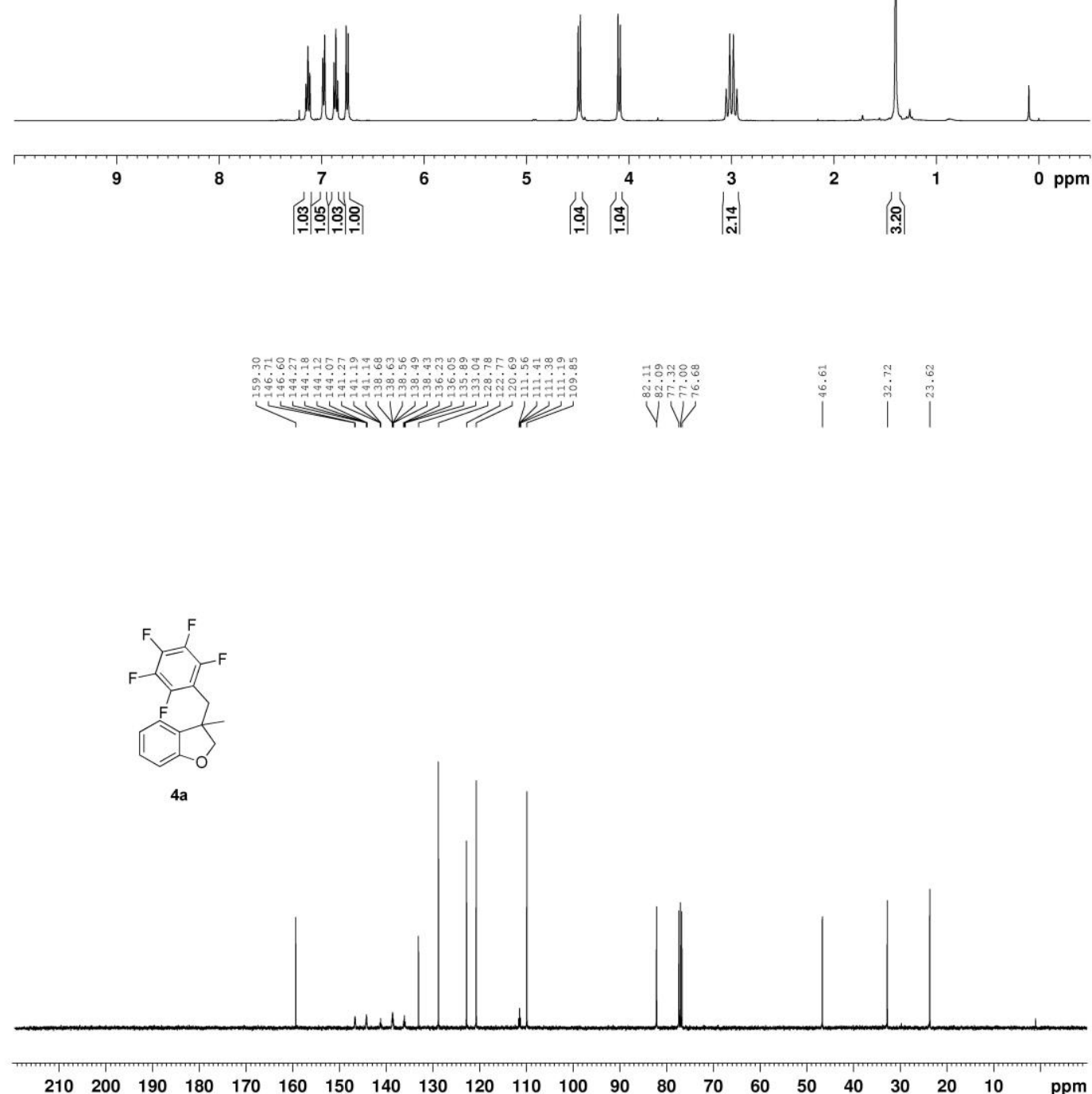


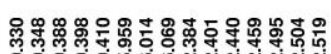

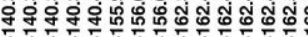

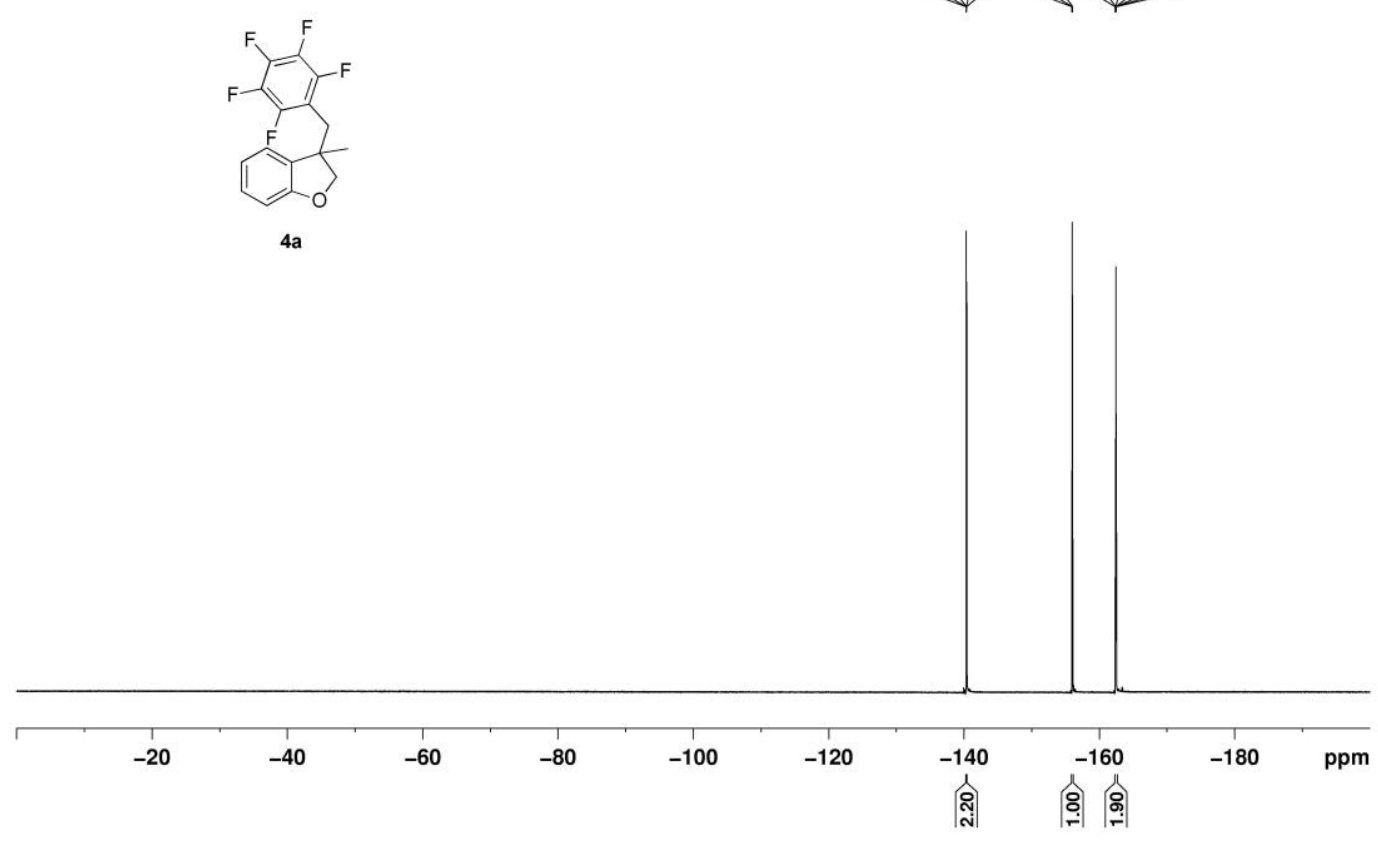

$4 a$

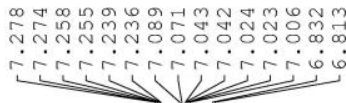

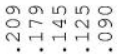

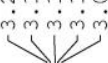

गे
ind

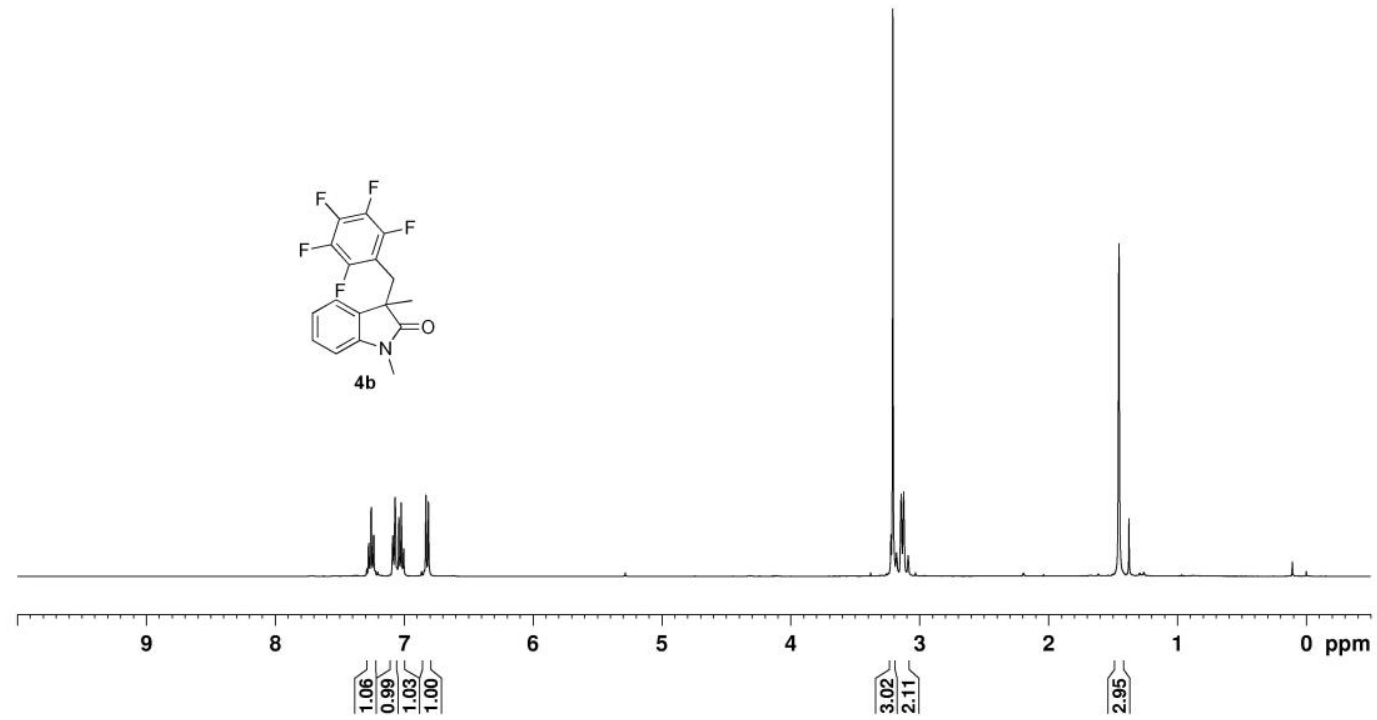



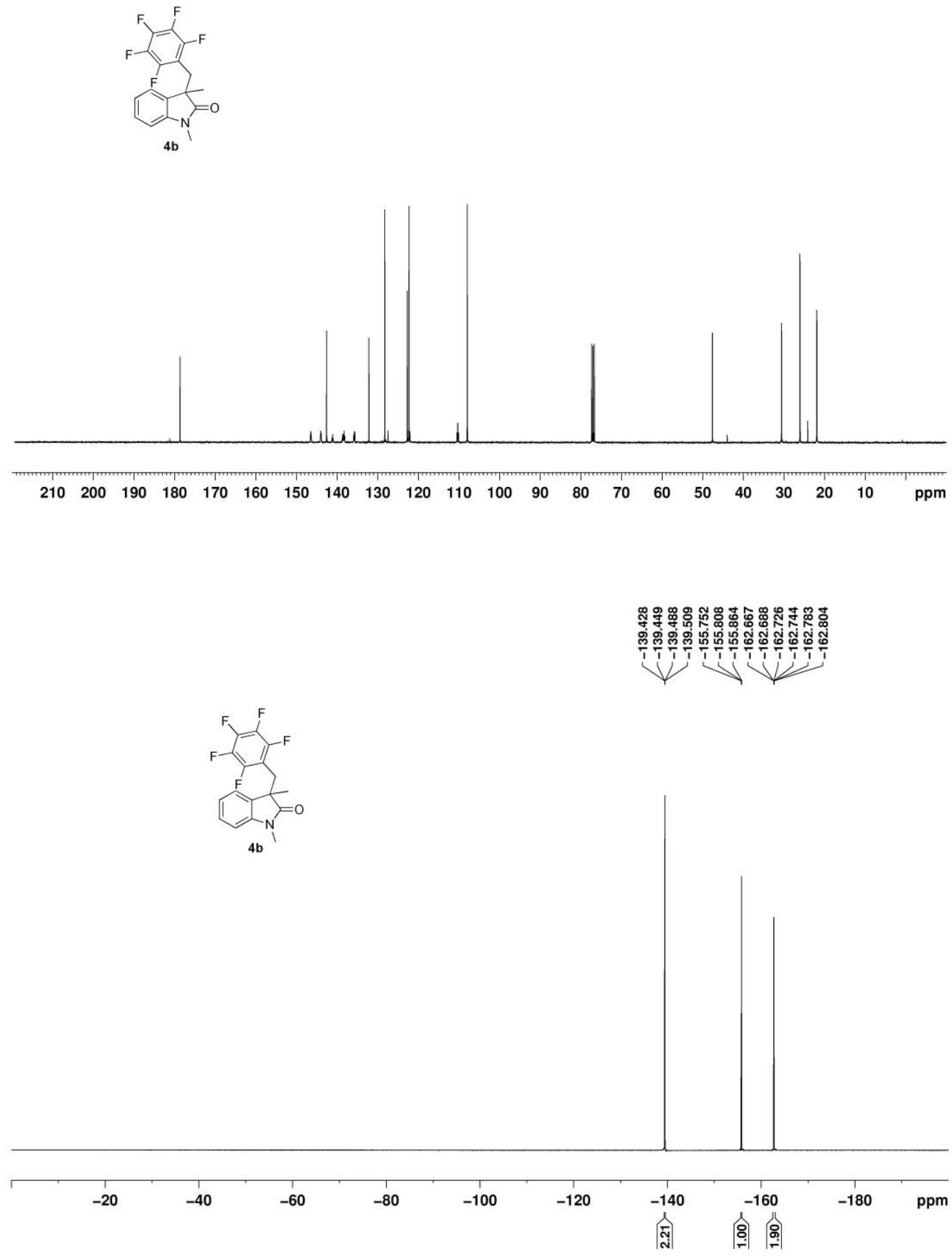

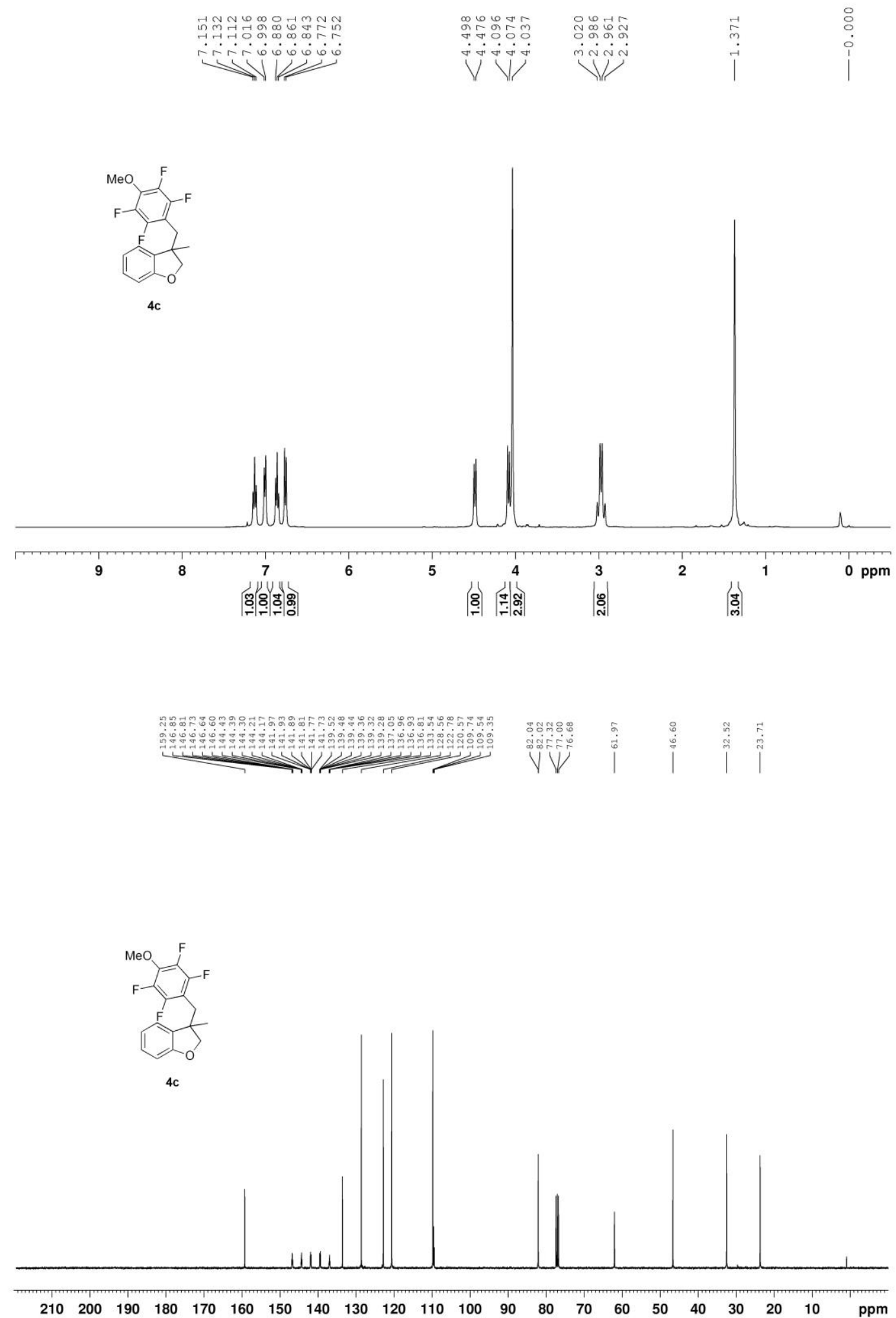


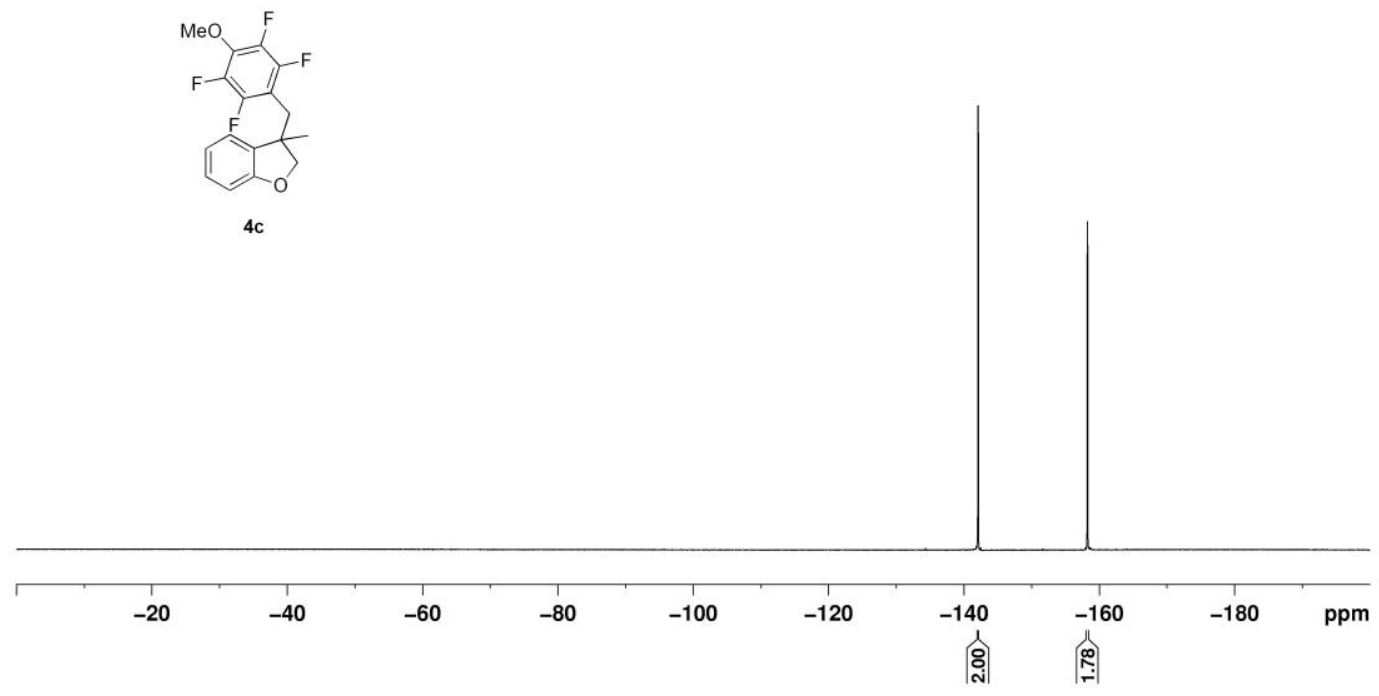

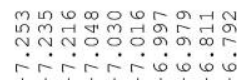

ivive

|

\begin{tabular}{ll}
$\infty$ & $\vdots$ \\
\hdashline & $\vdots$ \\
\hdashline & $\vdots$ \\
$i$ & $i$
\end{tabular}

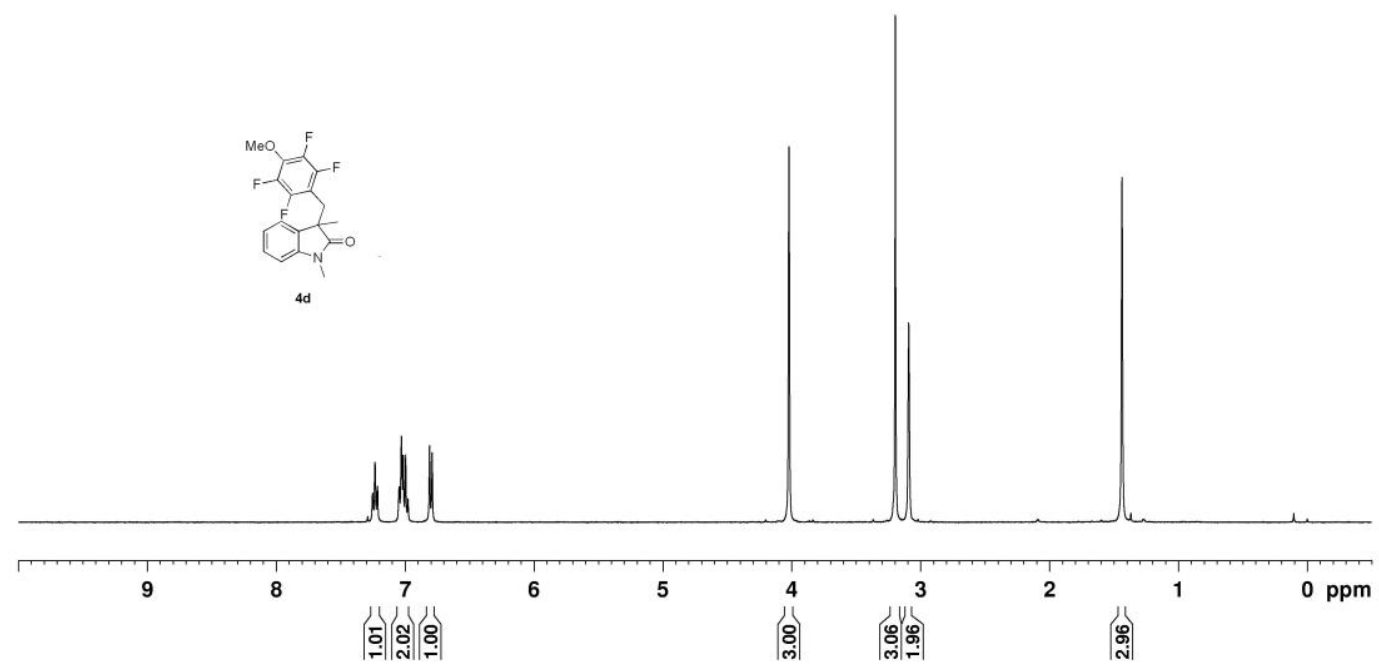




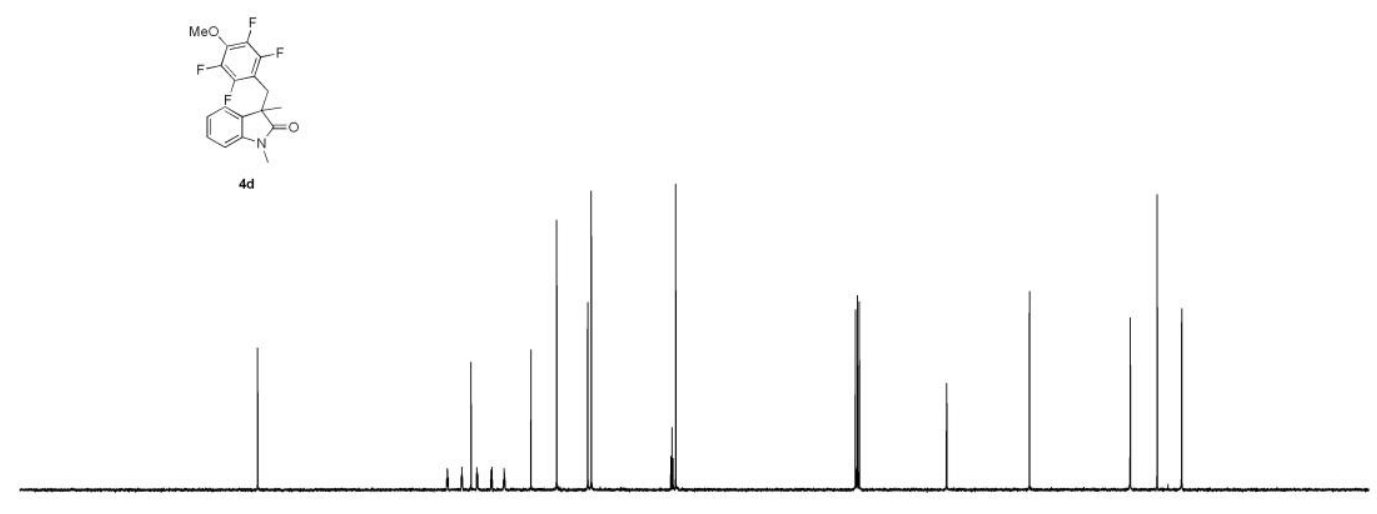

$\begin{array}{llllllllllllllllllllll}210 & 200 & 190 & 180 & 170 & 160 & 150 & 140 & 130 & 120 & 110 & 100 & 90 & 80 & 70 & 60 & 50 & 40 & 30 & 20 & 10 & \mathrm{ppm}\end{array}$

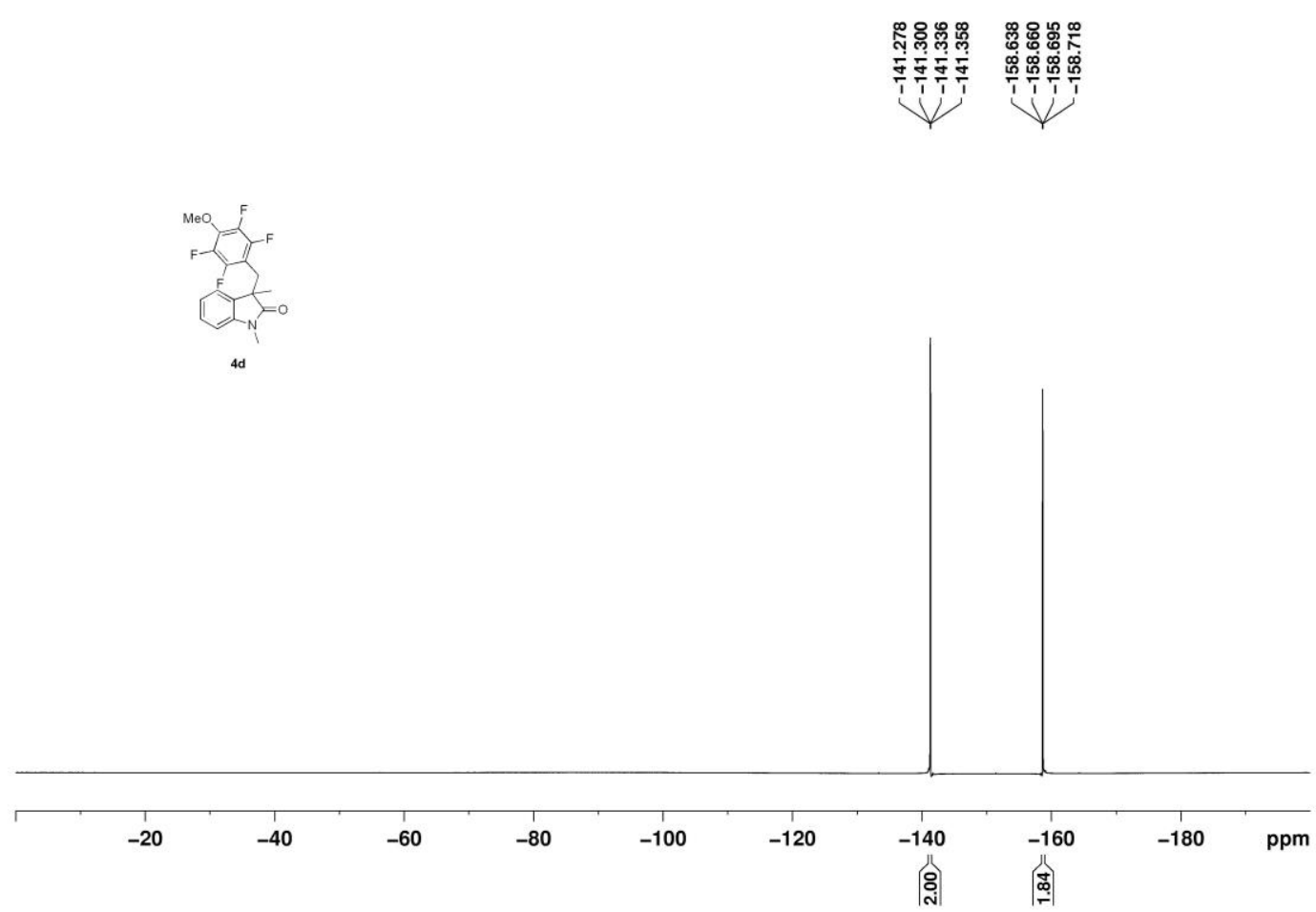



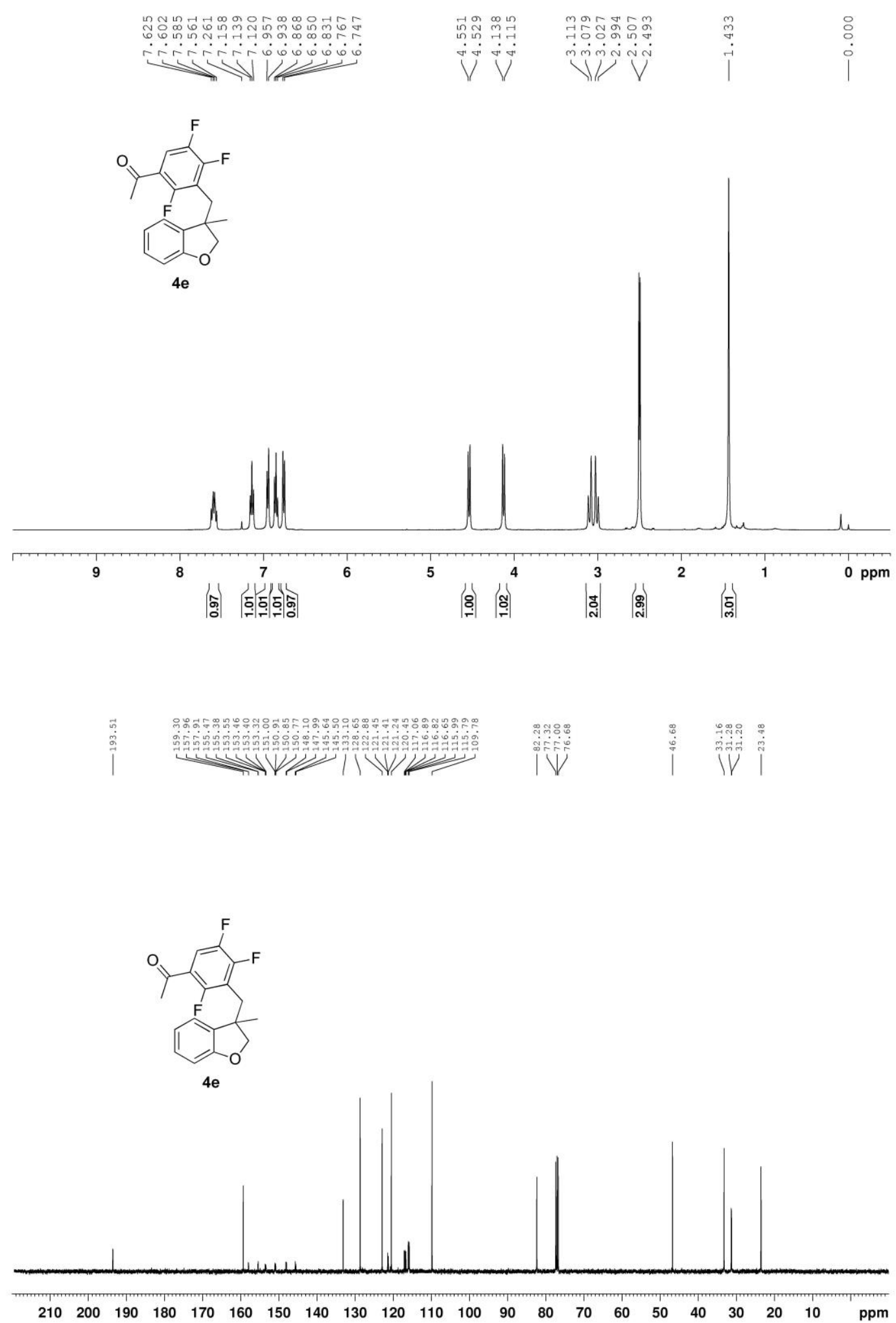


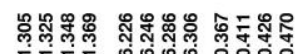

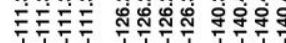

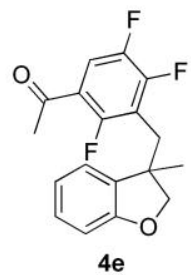

W W

$4 \mathrm{e}$

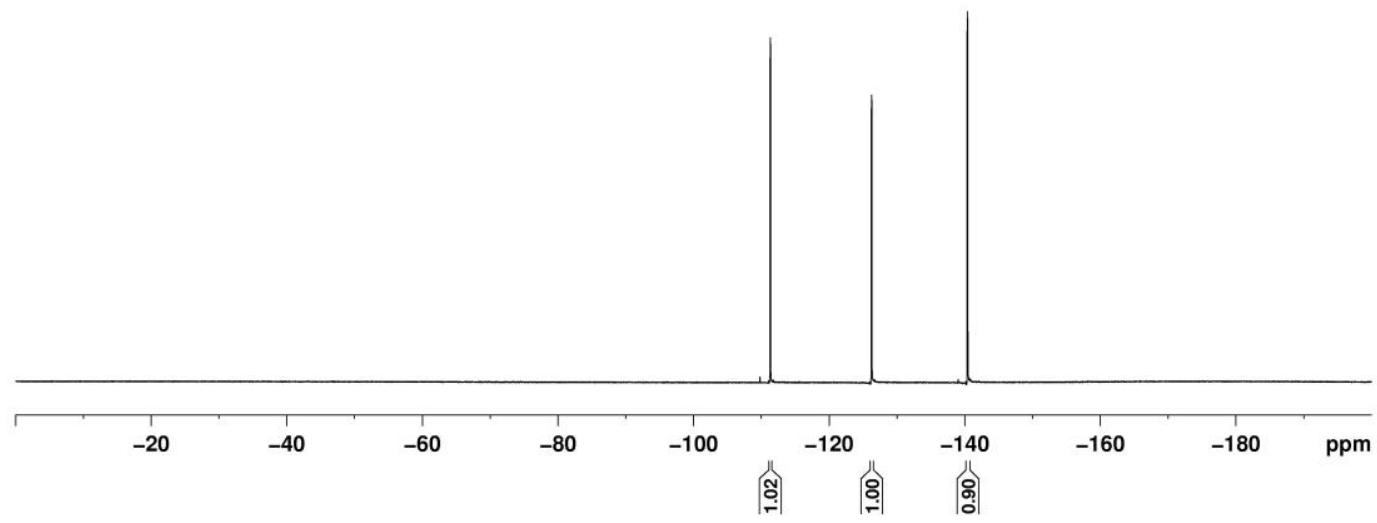

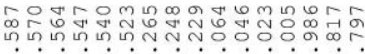

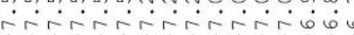

Vִ

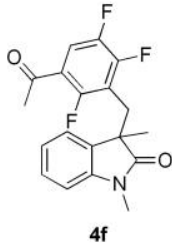

iill

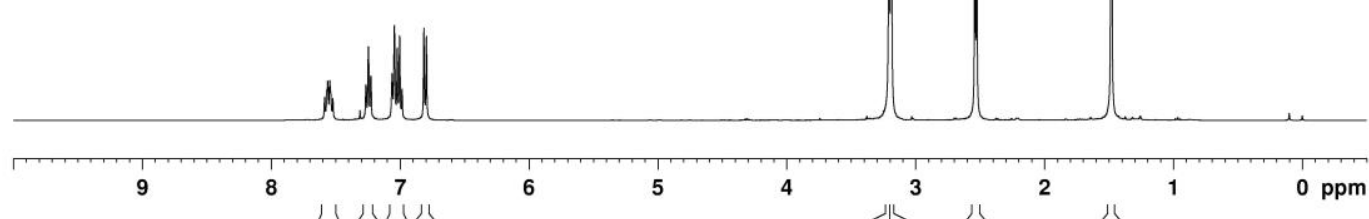

: :

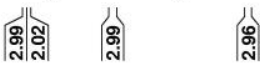



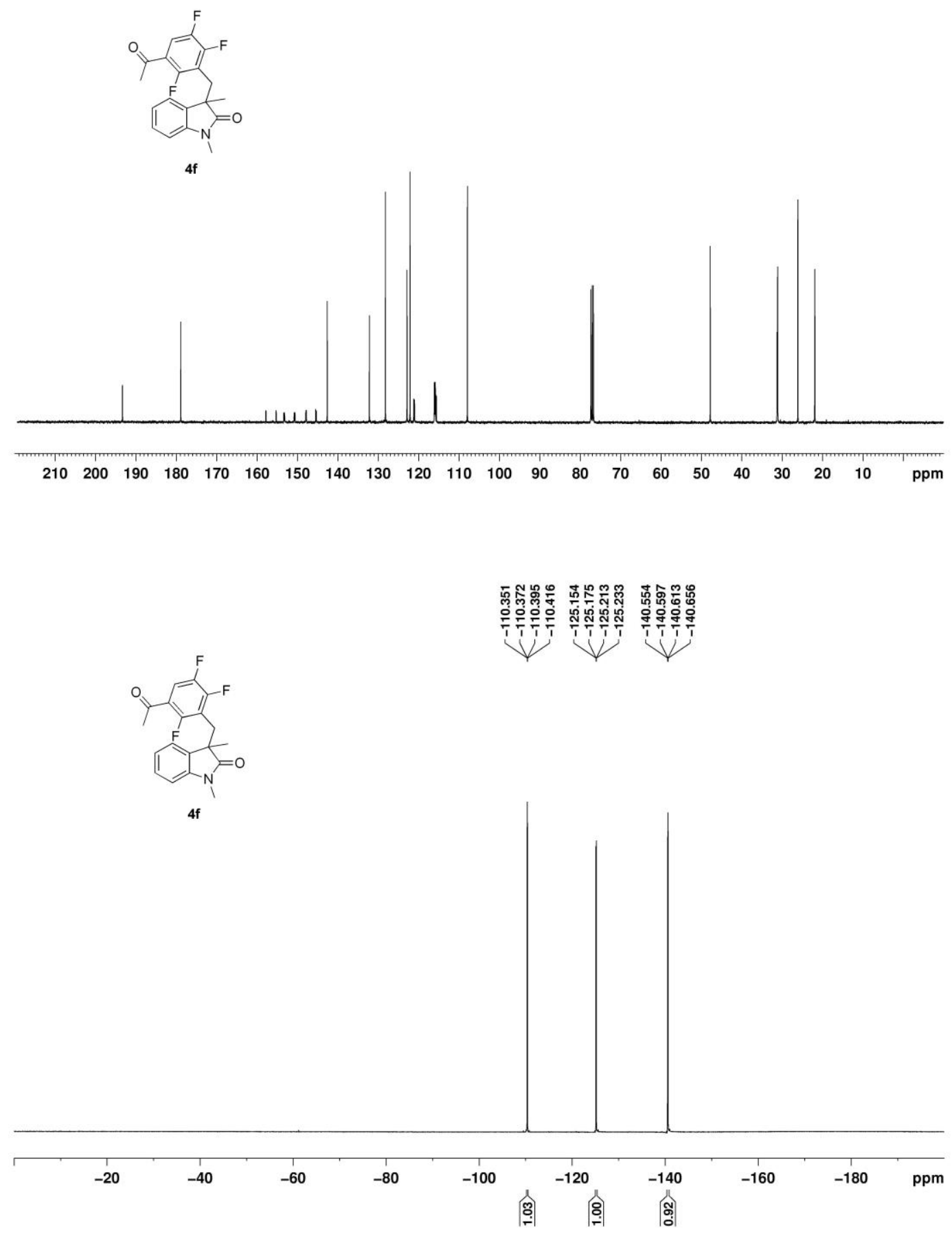


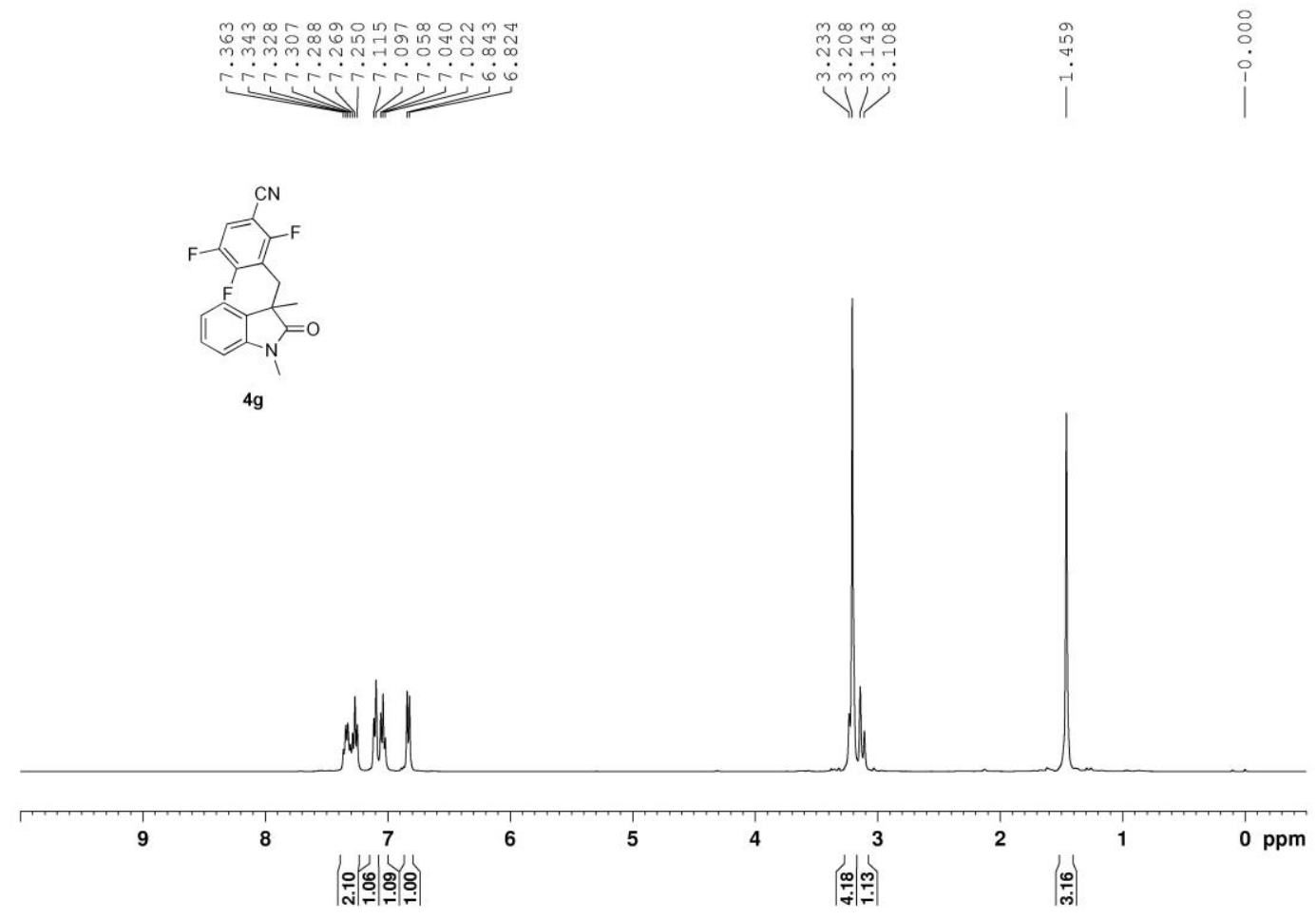

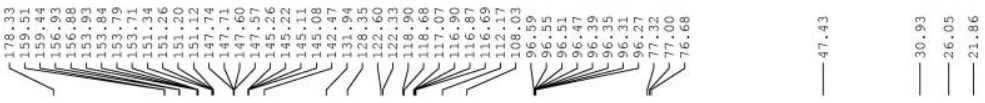

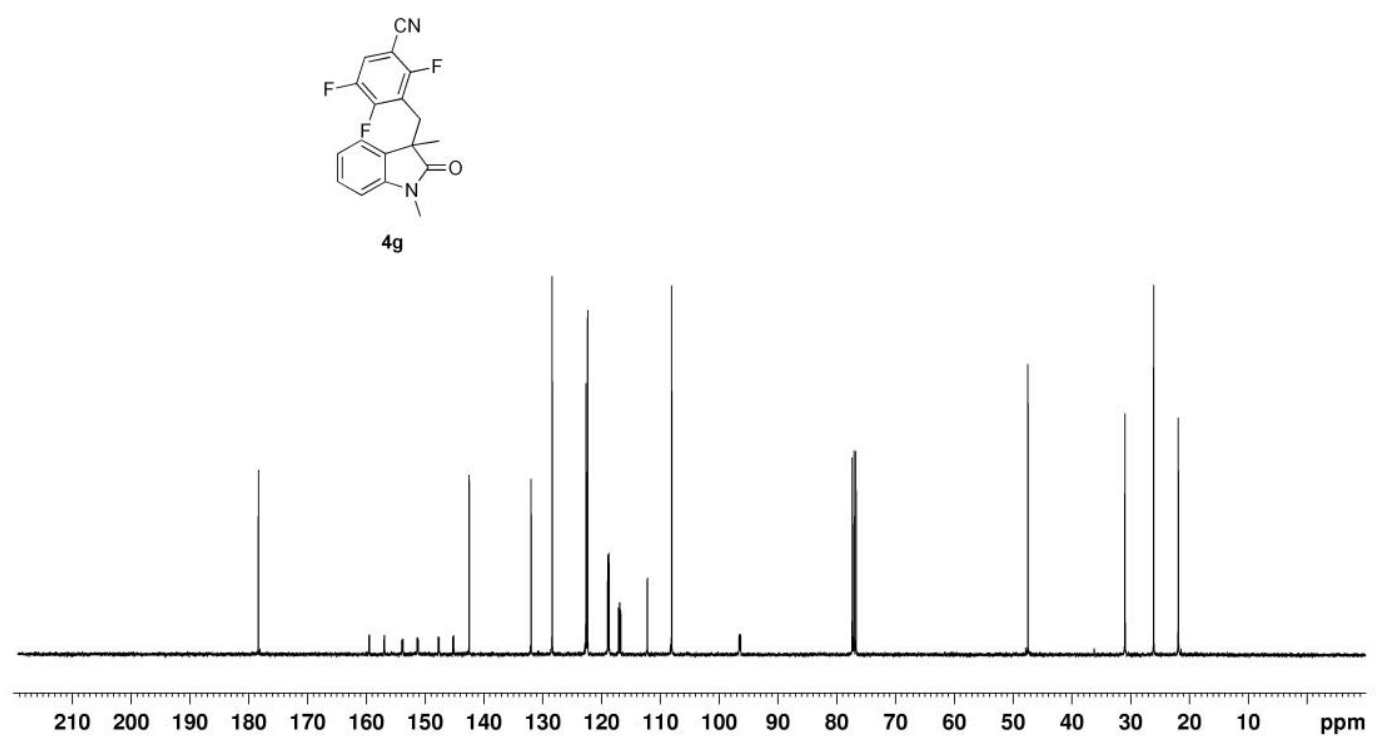




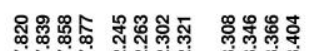

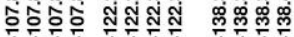

栲

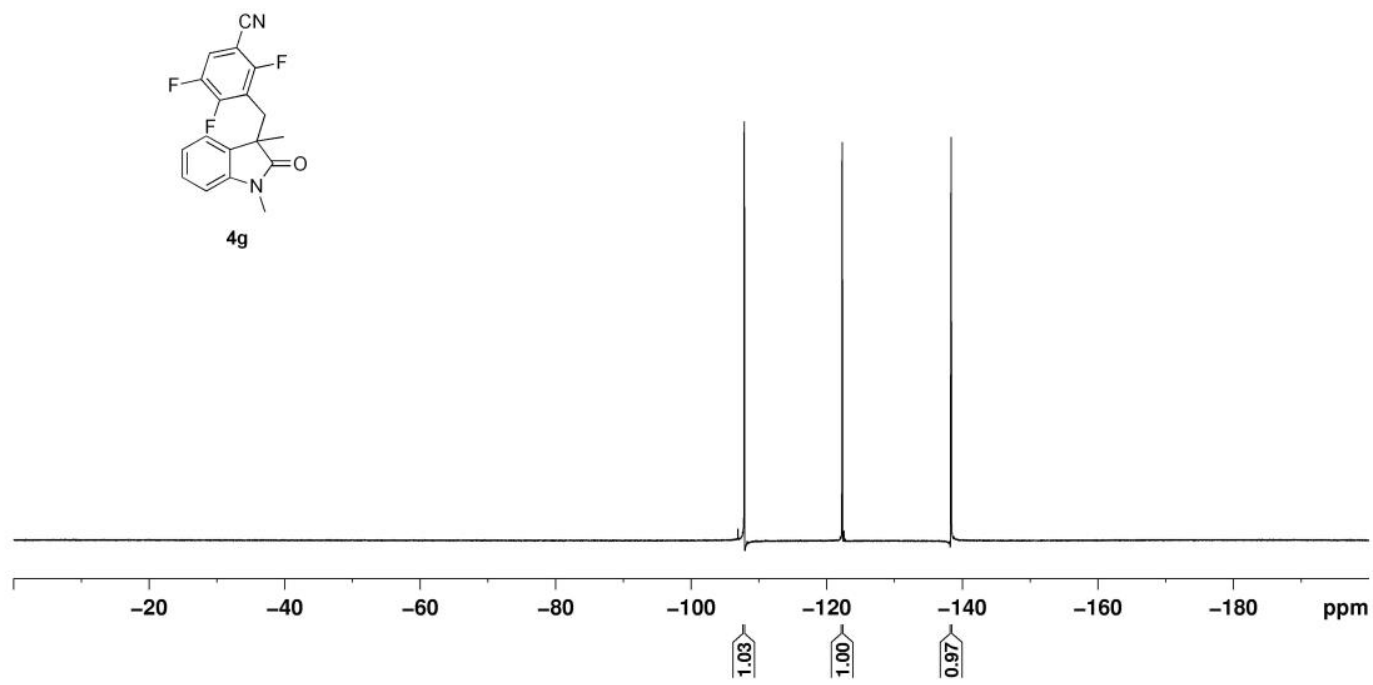

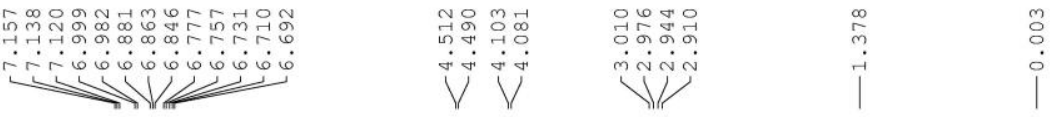

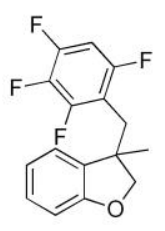

$4 h$

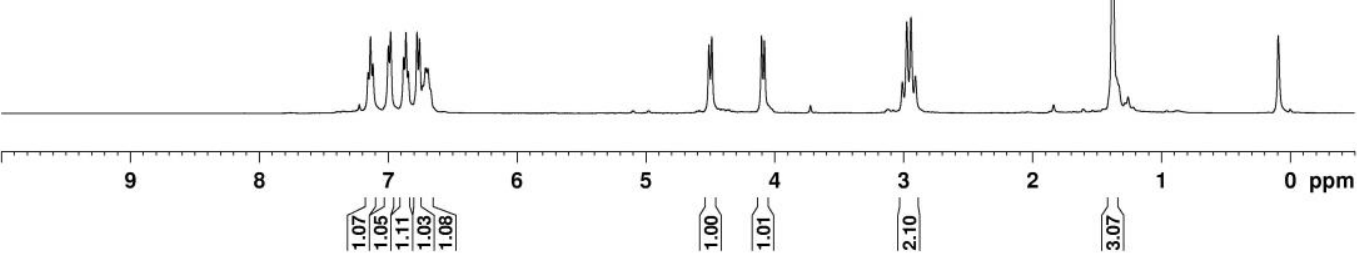



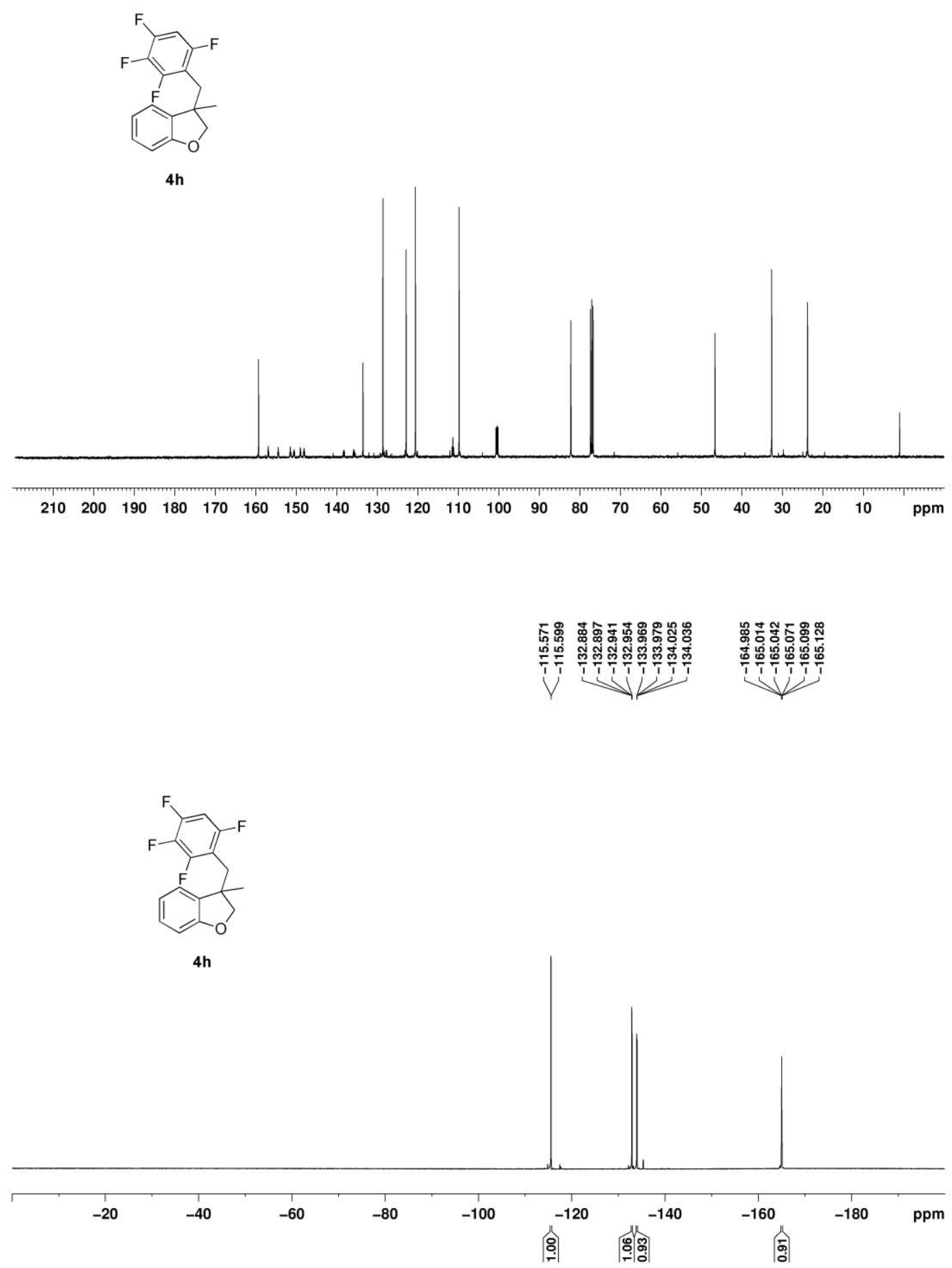

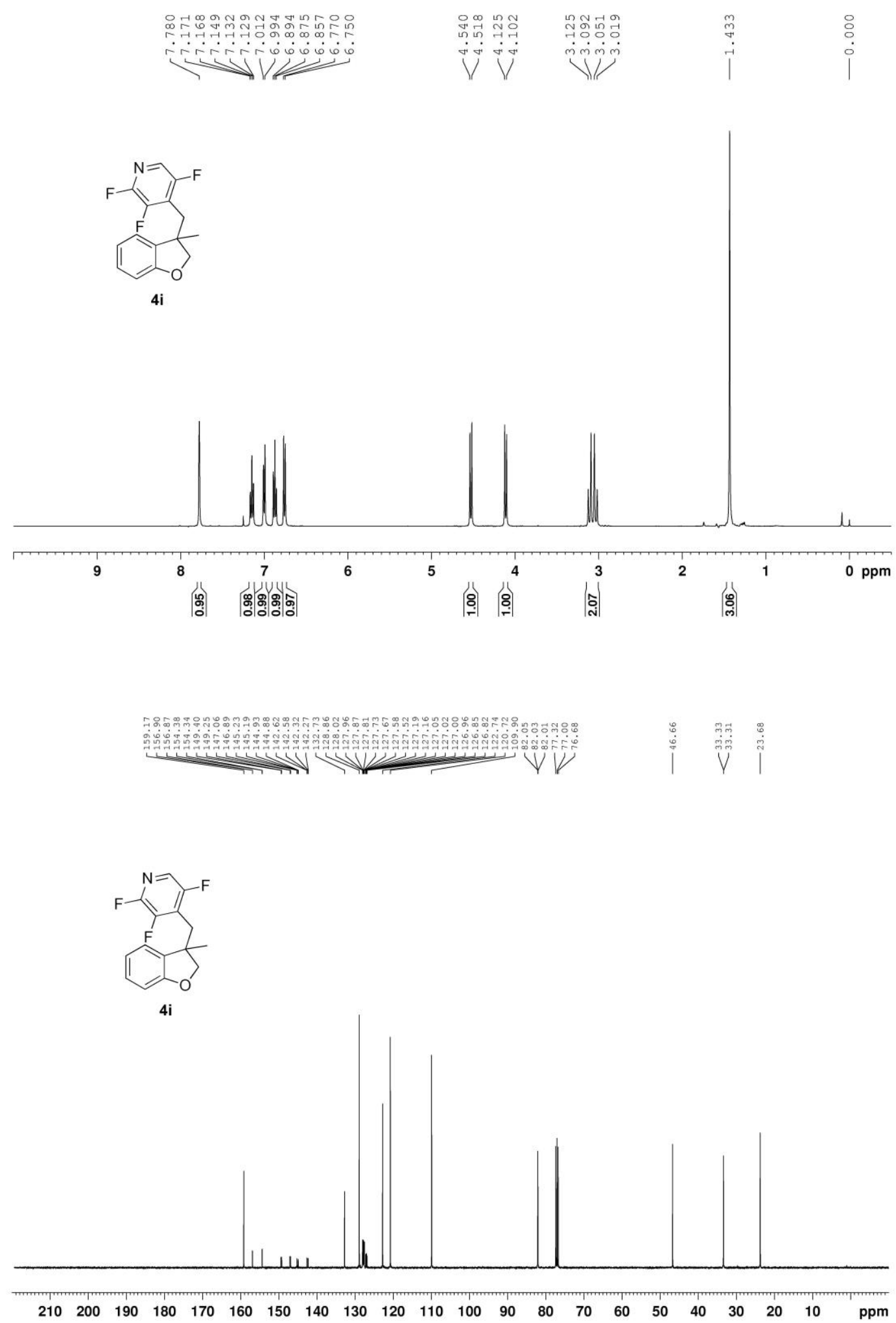

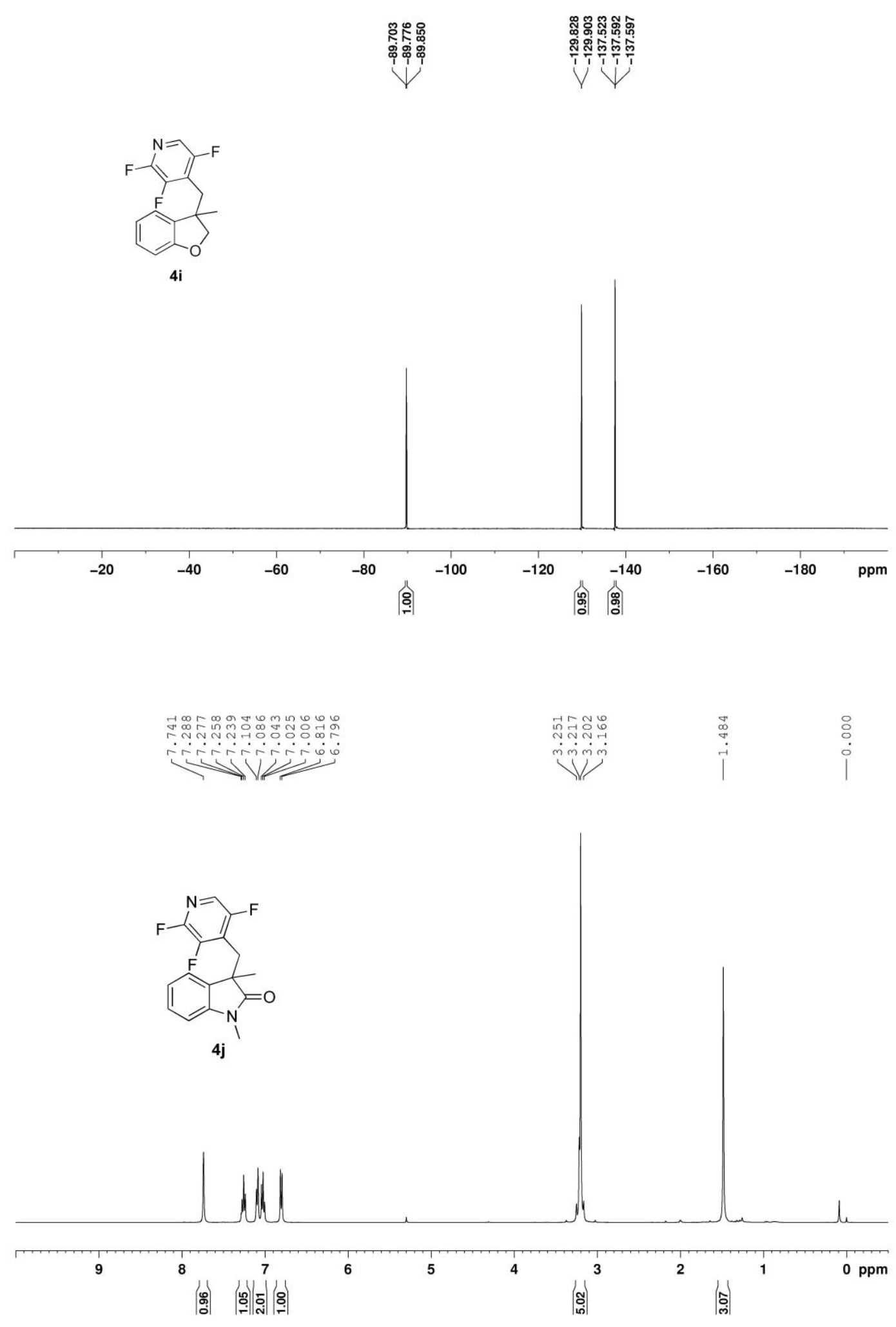
<smiles>CN1C(=O)C(C)(Cc2c(F)cnc(F)c2F)c2ccccc21</smiles>

4j
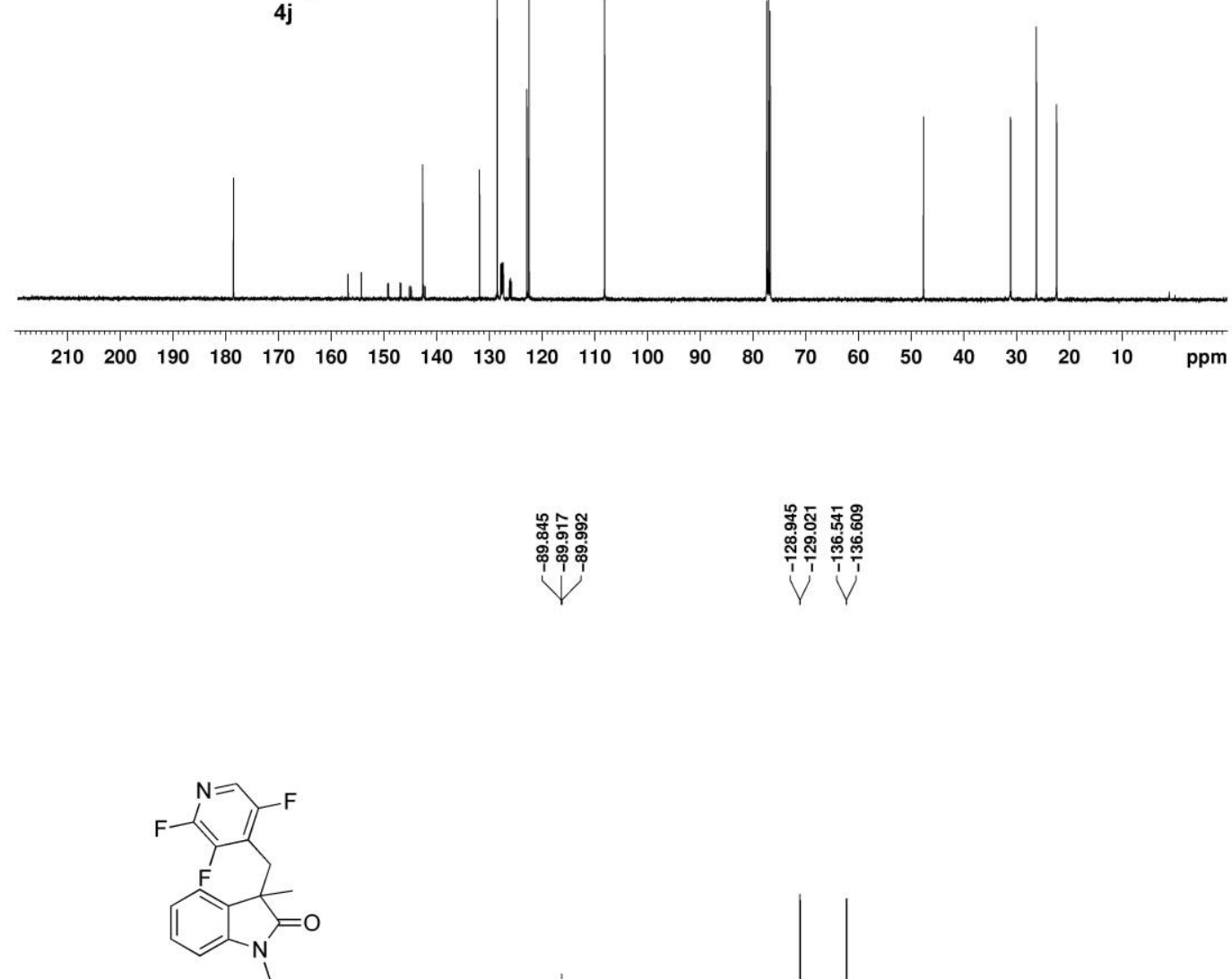

4j

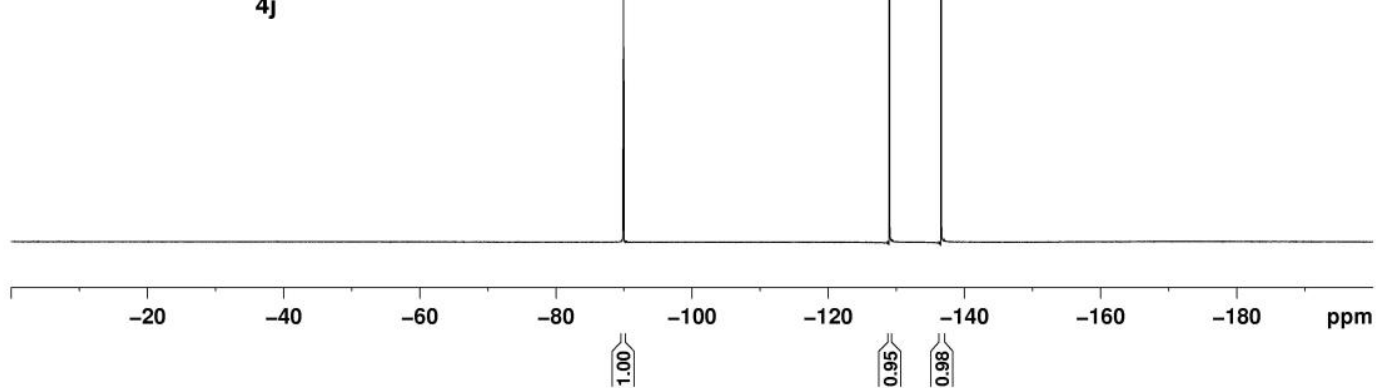




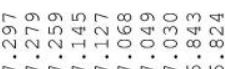

rinisingo

구ำ 글

मुलिल

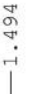

$\stackrel{\circ}{\circ}$

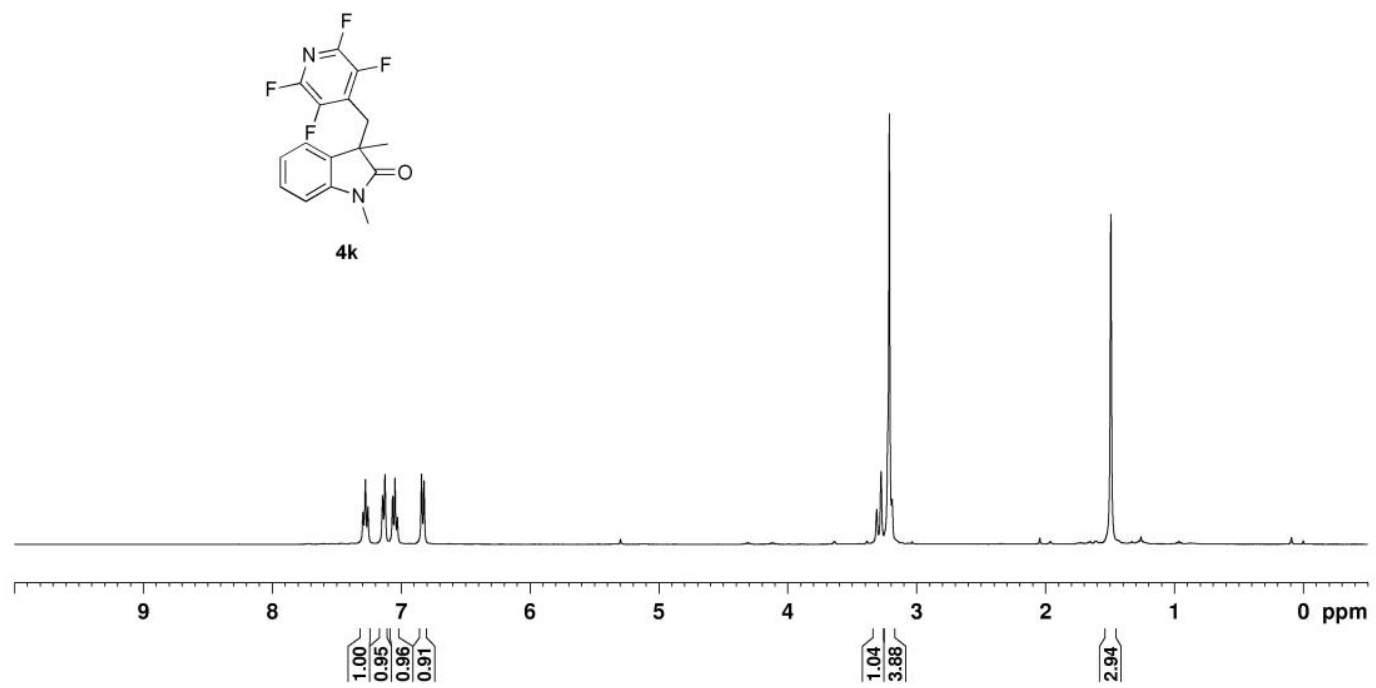

$4 k$

|े

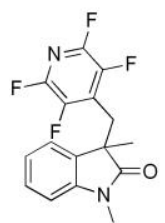

4k

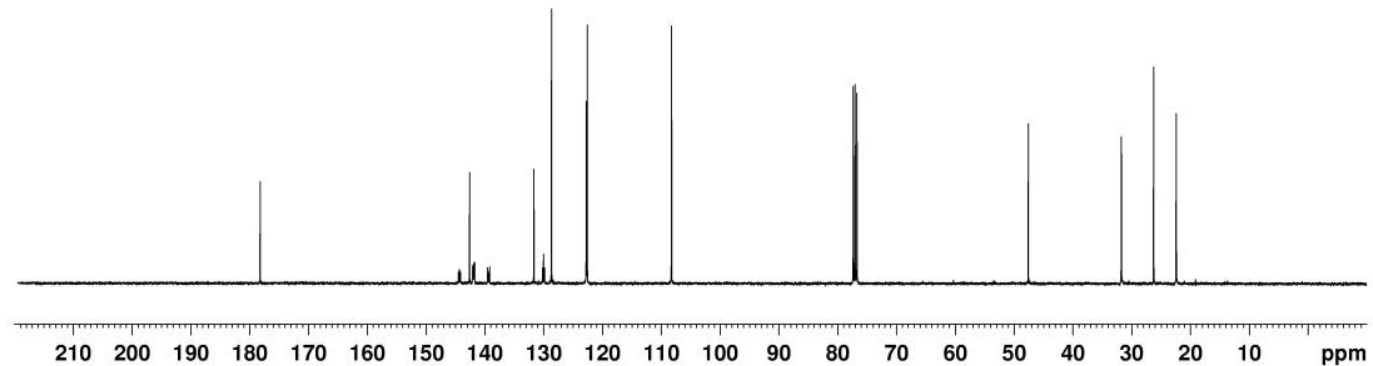



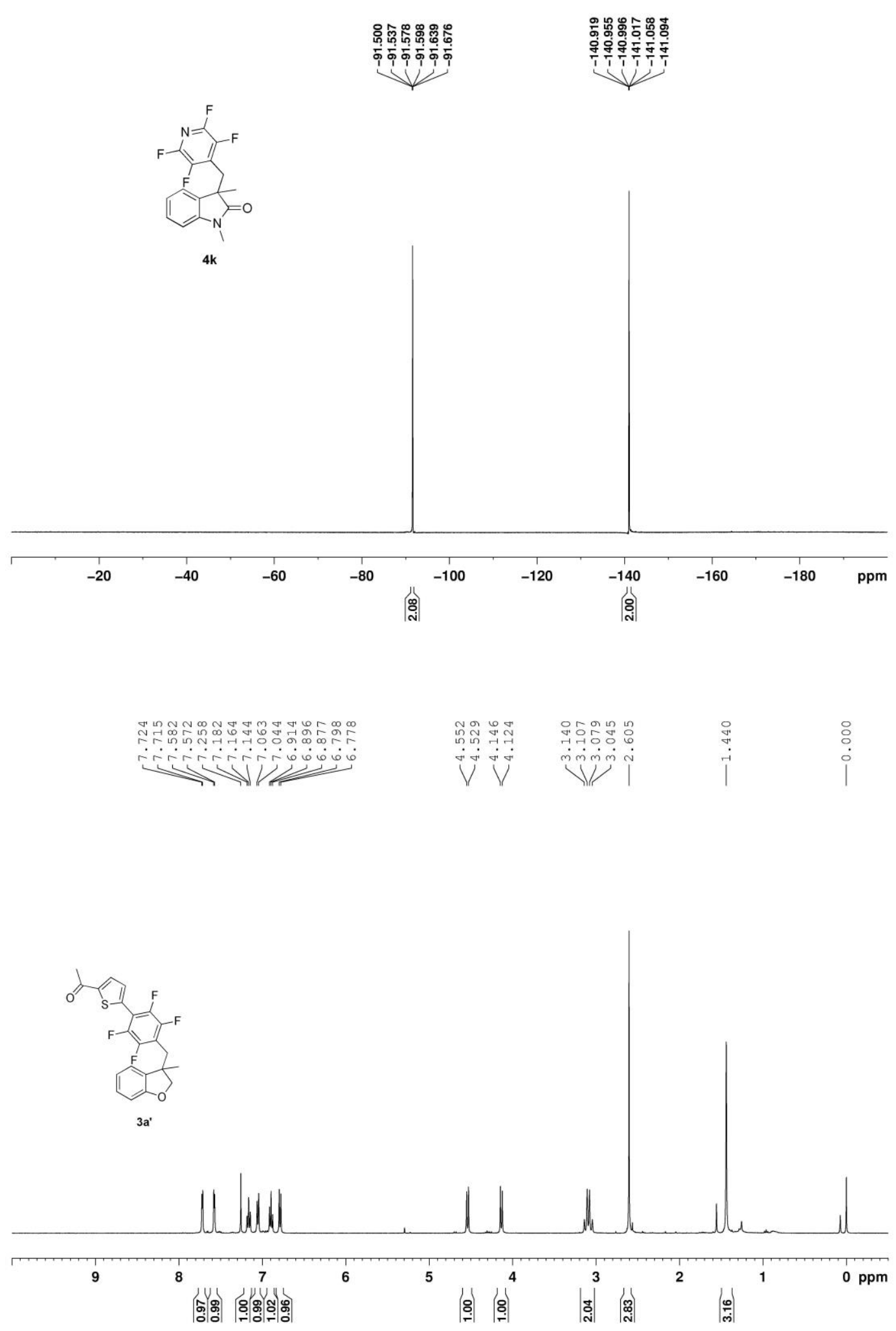

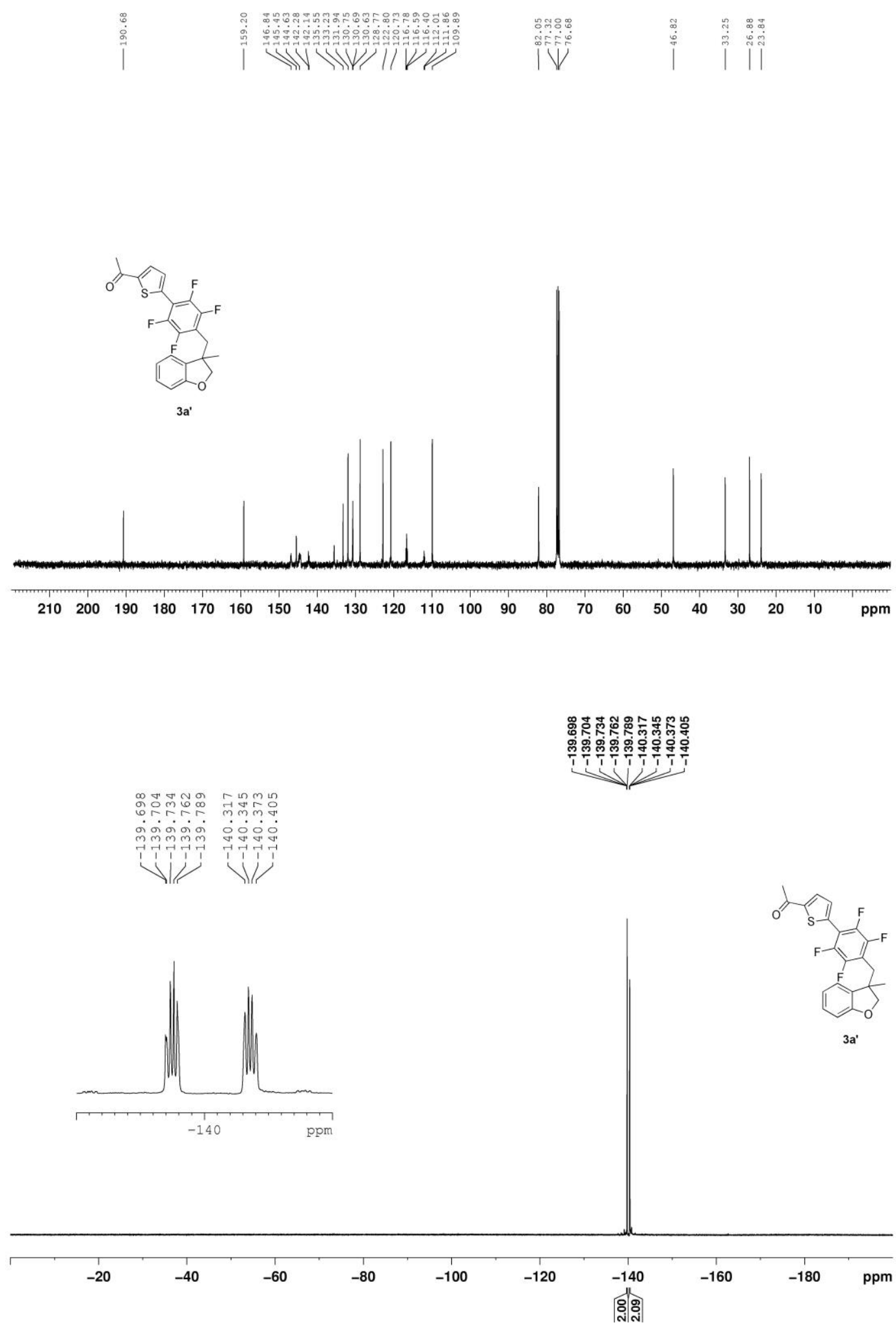

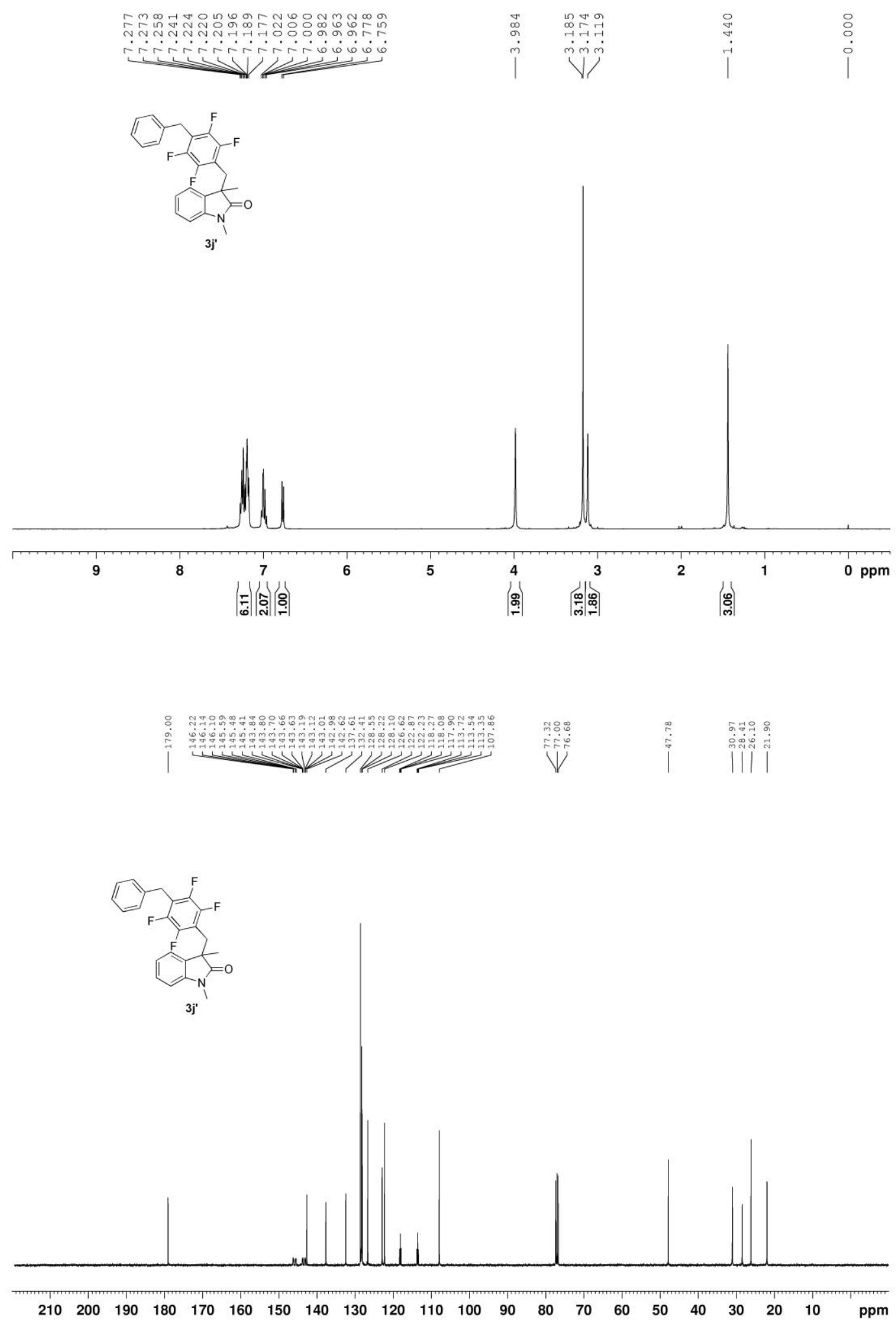


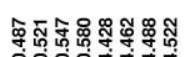

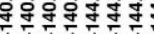
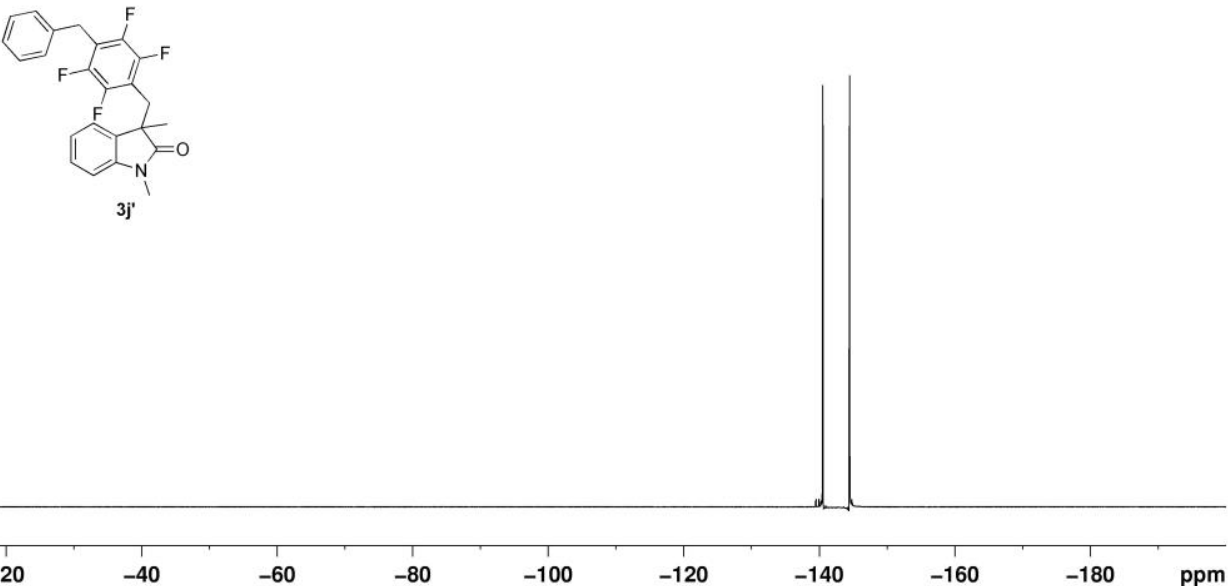

$-20$

$-40$

$-60$

$-80$

$-100$

수요

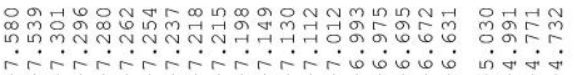

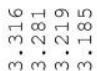
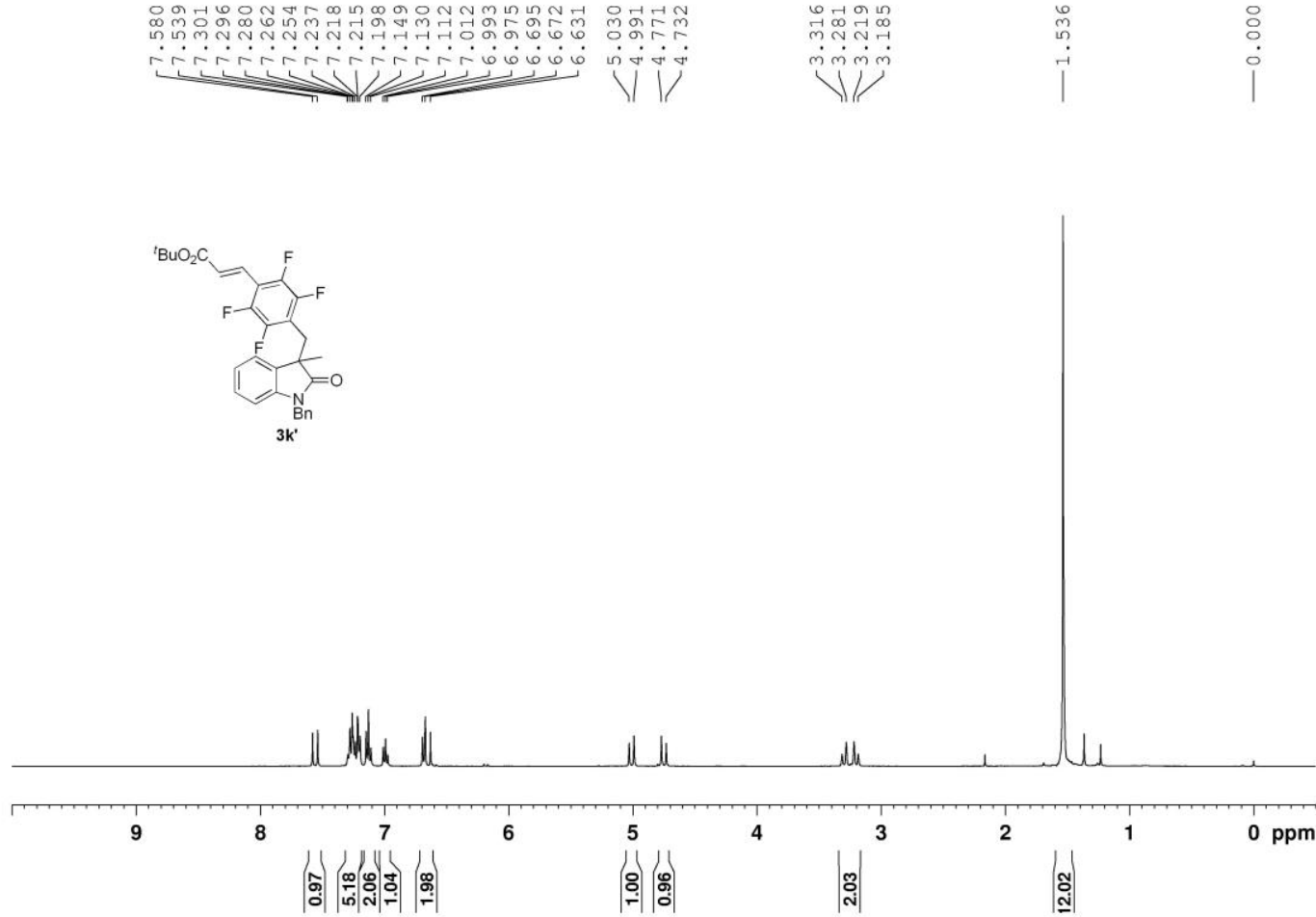

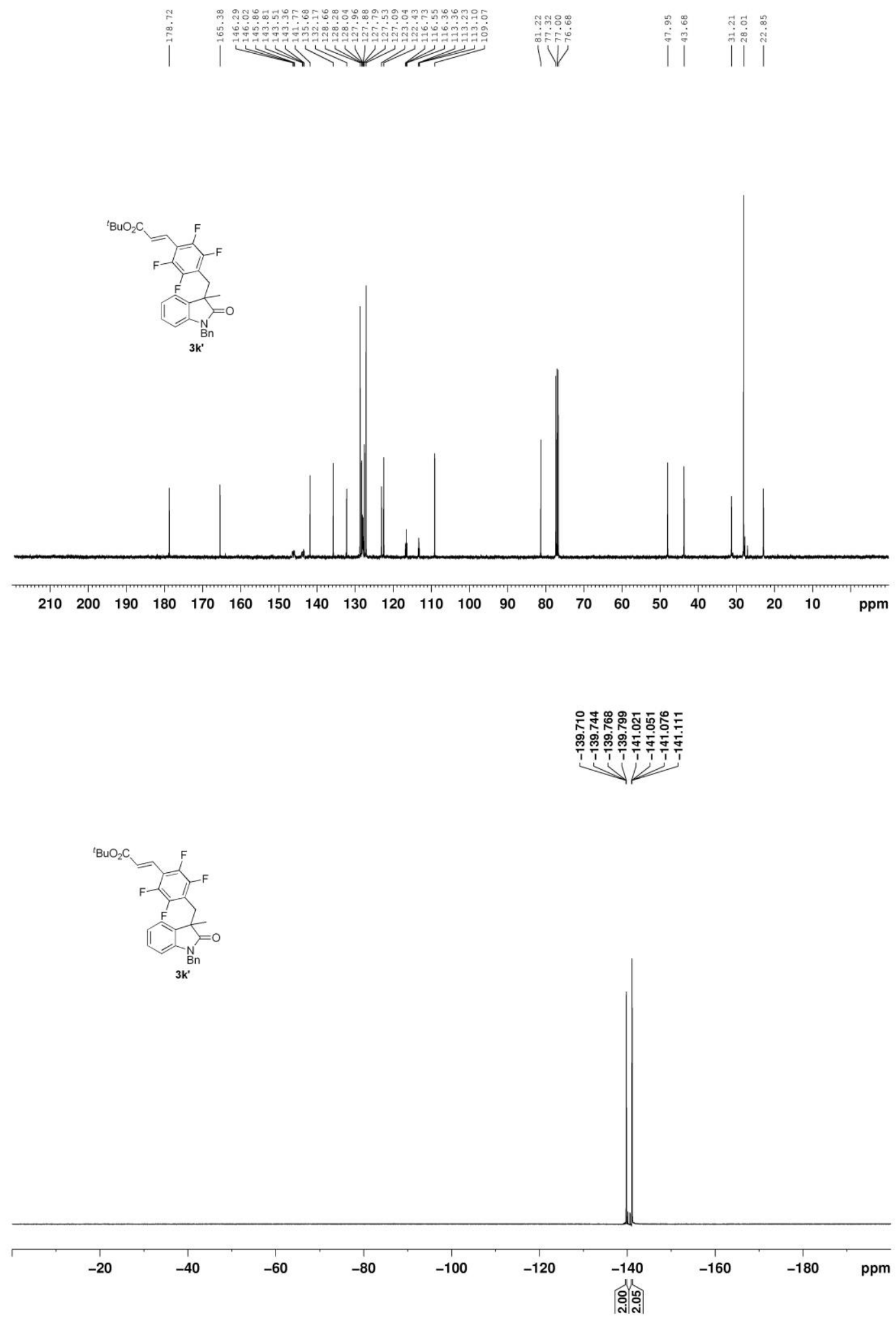